\title{
Antioxidant activity: from model to man
}

Citation for published version (APA):

Balk, J. M. (2010). Antioxidant activity: from model to man. [Doctoral Thesis, Maastricht University]. Maastricht University. https://doi.org/10.26481/dis.20100520jb

Document status and date:

Published: 01/01/2010

DOI:

10.26481/dis.20100520jb

Document Version:

Publisher's PDF, also known as Version of record

\section{Please check the document version of this publication:}

- A submitted manuscript is the version of the article upon submission and before peer-review. There can be important differences between the submitted version and the official published version of record.

People interested in the research are advised to contact the author for the final version of the publication, or visit the DOI to the publisher's website.

- The final author version and the galley proof are versions of the publication after peer review.

- The final published version features the final layout of the paper including the volume, issue and page numbers.

Link to publication

\footnotetext{
General rights rights.

- You may freely distribute the URL identifying the publication in the public portal. please follow below link for the End User Agreement:

www.umlib.nl/taverne-license

Take down policy

If you believe that this document breaches copyright please contact us at:

repository@maastrichtuniversity.nl

providing details and we will investigate your claim.
}

Copyright and moral rights for the publications made accessible in the public portal are retained by the authors and/or other copyright owners and it is a condition of accessing publications that users recognise and abide by the legal requirements associated with these

- Users may download and print one copy of any publication from the public portal for the purpose of private study or research.

- You may not further distribute the material or use it for any profit-making activity or commercial gain

If the publication is distributed under the terms of Article $25 \mathrm{fa}$ of the Dutch Copyright Act, indicated by the "Taverne" license above, 
Antioxidant activity:

from model to man 
(C) J.M. Balk, Maastricht 2010

ISBN 978-90-5335-269-4

Cover design and layout: Simone Vinke, Ridderprint, Ridderkerk, The Netherlands Printed by: Ridderprint, Ridderkerk, The Netherlands

The printing of this thesis was financially supported by Zambon Nederland B.V., and HERO Nederland B.V. 


\title{
Antioxidant activity: from model to man
}

\author{
PROEFSCHRIFT \\ Ter verkrijging van de graad van doctor \\ aan de Maastricht University, \\ op gezag van de Rector Magnificus, \\ Prof. mr. G.P.M.F. Mols, \\ volgens het besluit van het College van Decanen, \\ in het openbaar te verdedigen \\ op donderdag 20 mei 2010 om 16.00 uur
}

door

Jiska Mirjam Balk 


\section{Promotor:}

Prof. dr. A. Bast

\section{Co-promotor:}

Dr. G.R.M.M. Haenen

\section{Beoordelingscommissie:}

Prof. dr. J. Rosing (voorzitter)

Dr. C.T.A. Evelo

Prof. dr. E.C.M. Mariman

Prof. dr. ir. H. Gruppen (Wageningen University)

Dr. M. Stefek (Slovak Academy of Sciences, Bratislava, Slovakia) 
If people do not believe that mathematics is simple, it is only because they do not realize how complicated life is. John von Neumann (1947) 



\section{Contents}

Chapter $1+9$

Introduction

Chapter 2

Evaluation of the accuracy of antioxidant competition assays.

Incorrect assumptions with major impact

Chapter 3

Prevention of a systematic underestimation of antioxidant activity in competition assays.

The impact of unspecific reactions of reactive species

Chapter 4

61

Stoichiometry and capacity versus reaction rate in antioxidant activity.

Not the reaction rate, but the number of reactive species scavenged determines

the observed antioxidant activity

Chapter 5

Increased citrate levels in non-alcoholic steatohepatitis: effect on CYP2E1

Chapter 6

Substrate inhibition of CYP2E1 activity is primarily determined by lipophilicity

Chapter 7

Difference in effect of apples, fruit salad and fruit beverage on uric acid concentration and total plasma antioxidant capacity

Chapter 8

Discussion

Algemene samenvatting

Dankwoord

Curriculum Vitae 
$d x_{1}=-k_{j} \cdot x_{1}$
$d x_{2}=k_{0} w_{1}$ 


\section{Chapter 1}

Introduction

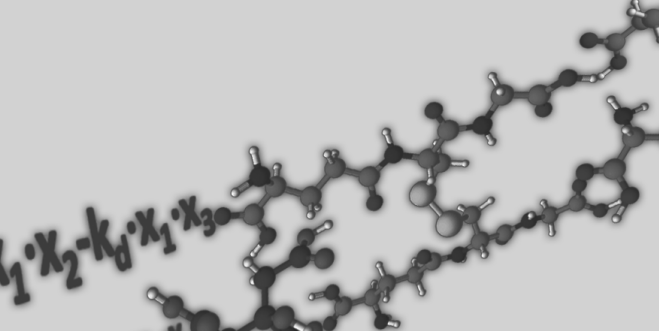

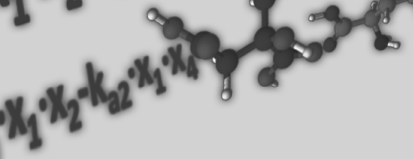





\section{The chemistry behind reactive species}

From a biological perspective, oxygen $\left(\mathrm{O}_{2}\right)$ is a peculiar molecule. On the one hand, oxygen is indispensable in aerobic life. The lack of oxygen in man for only a few minutes results in severe organ damage or even death. On the other hand, oxygen is also a toxic molecule. Exposure to high concentrations of oxygen can result in irreversible damage to for example the central nervous system and the lungs [1, 2].

This ambivalent character of the oxygen molecule is caused by its chemical nature. Molecular oxygen is constructed from two oxygen atoms, each with six valence electrons. Ten electrons completely fill the $\sigma_{s}$ bonding and anti-bonding molecular orbitals and the $\sigma_{p^{\prime}} \pi_{x}$ and $\pi_{y}$ bonding orbitals (figure 1). Two electrons occupy the $\pi_{x}$ and $\pi_{y}$ anti-bonding orbitals. According to Hund's rule of maximum multiplicity, the lowest energy state of the molecule is the configuration in which each electron occupies one anti-bonding orbital and their spins are parallelly aligned. The resulting spin of one (twice the spin of $1 / 2$ of each parallel electron) indicates that oxygen, in its most stable energy state, is in a triplet state: triplet oxygen (denoted as ${ }^{3} \mathrm{O}_{2}$ ).

The two electrons in the anti-bonding orbitals of triplet oxygen are unpaired electrons, which makes triplet oxygen a biradical. Radicals are in general highly reactive molecules, since they easily donate one electron (oxidation) to another molecule, or accept one electron (reduction) from the other molecule. Among the radicals generated in the human body are the hydroxyl radical $(\cdot \mathrm{OH})$, the nitric oxide radical (NO) and superoxide anion $\left(\mathrm{O}_{2}^{-}{ }^{-}\right.$, with three electrons in the anti-bonding orbitals). Radicals, like $\cdot \mathrm{OH}$, can react with virtually any biological compound including poly-unsaturated fatty acids (resulting in deterioration of the membrane structure), proteins (resulting in dysfunction of the protein) and DNA (resulting in genomic instability) [3].

Despite its biradical configuration, triplet oxygen is relatively unreactive. Due to the rule of spin conservation, triplet oxygen can only react with molecules that are also in the triplet state (resultant spin of one). In most compounds all electrons are paired, which indicate that they are not in a triplet state. In biological systems, only a few molecules will be able to react with triplet oxygen, like haem containing proteins [4]. The spin conservation limits the reactivity of the biradical triplet oxygen.

Oxygen can however also be in a singlet state. In this state, the two electrons that occupy the antibonding orbitals are aligned anti-parallel, resulting in a resultant spin of zero. These two electrons can occupy one anti-bonding orbital each, or both electrons are paired in one anti-bonding orbital (leaving the other anti-bonding orbital empty).

If both anti-bonding orbitals contain one electron each, this singlet state of oxygen is also a biradical (figure 1). This configuration is however highly unstable and it quickly changes to the configuration with two electrons occupying one anti-bonding orbital, which is referred to as singlet oxygen $\left({ }^{1} \mathrm{O}_{2}\right)$. The biradical configuration is the excited state of singlet oxygen $\left({ }^{1} \mathrm{O}_{2}^{*}\right)[5]$. 

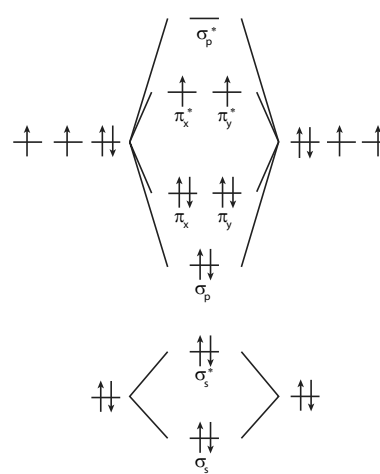

므- $-\underline{\bar{o}}$.

Triplet oxygen
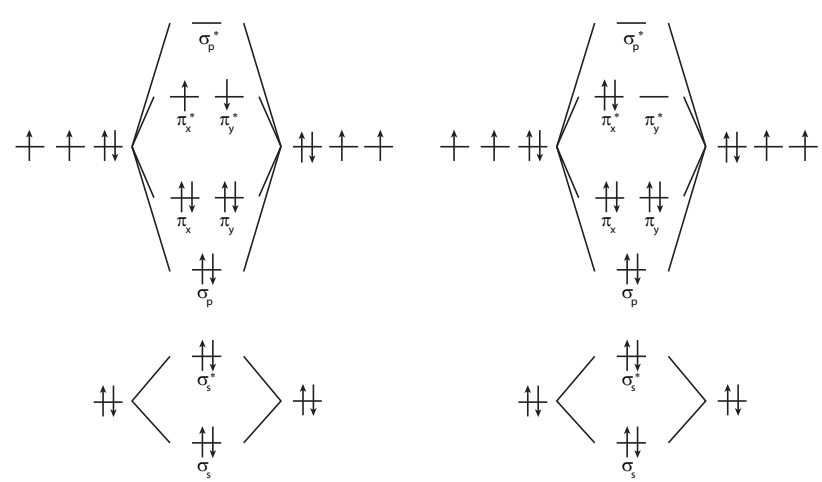

. $\overline{0}-\overline{\overline{0}}$.

Singlet oxygen excited state

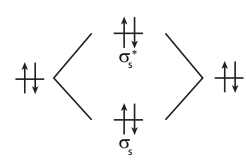

므$-\underline{\overline{0}}$

Singlet oxygen

Figure 1: The energy level diagrams of the three different electronic states of molecular oxygen with their Lewis structure.

Energetically, singlet oxygen is a less favorable configuration than triplet oxygen. The relaxation of singlet oxygen to triplet oxygen is however spin forbidden (rule of spin conservation). The relaxation requires the inversion of the spin of a single electron, which is a relatively slow process (microseconds to milliseconds). Instead of relaxation to the triplet state, singlet oxygen can easily accept two paired electrons that will occupy the empty anti-bonding orbital. These two paired electrons can be obtained easily from double bounds ( $\pi$-bounds) of other compounds and cyclic peroxide is formed. If oxygen, either in singlet or triplet configuration, is reduced by one electron the superoxide anion $\left(\mathrm{O}_{2}{ }^{-}\right)$is formed. Superoxide is both an oxidizer and a reductor. If it donates one electron to another molecule, triplet oxygen is formed. If superoxide is reduced by an extra electron, $\mathrm{O}_{2}^{2-}$ is formed, which upon protonation yields hydrogen peroxide $\left(\mathrm{H}_{2} \mathrm{O}_{2}\right)$. Hydrogen peroxide is a relatively stable molecule.

From $\mathrm{H}_{2} \mathrm{O}_{2}$, hypochlorous acid can be formed ( $\mathrm{HOCl}$, or the deprotonated form hypochlorite, OCl; $\mathrm{pK}_{\mathrm{a}}=7.5$ ) by the enzyme myeloperoxidase. $\mathrm{HOCl}$ has 14 valence electrons in total, which fill the bonding orbitals completely. $\mathrm{HOCl}$ is however a reactive species, which reactivity is mostly directed to thiol containing molecules in biological systems. At physiological $\mathrm{pH}$, the thiol is partially deprotonated, resulting in a negative charge located at the sulfur atom. This negatively charged sulfur creates a bond with a slightly positively charged chlorine atom (due to the difference in electronegativity of the oxygen atom and the chlorine atom). A sulfur-chloride bond is formed, resulting in the formation of the hydroxyl anion [6]. The sulfur chloride is converted to a sulfenic acid by a reaction of a hydroxyl anion with the sulfur [7].

The reactive species peroxynitrite is formed out of the reaction of the radicals superoxide and nitric oxide. In physiological conditions, both the protonated form (peroxynitrous acid) and the deprotonated form (peroxynitrite anion) are present (both denoted as peroxynitrite). Peroxynitrite can be an oxidant performing one-electron and two-electron reductions, but it can also easily 
dissociate to secondary radicals like hydroxyl radicals and nitrogen dioxide [8]. In the presence of carbon dioxide, peroxynitrite can form a complex which yields carbonate radicals and nitrogen dioxide [8].

Once one of these reactive species is produced, it can easily spread its reactivity to the biological environment giving rise to secondary radicals like tyrosine radicals, lipid radicals and lipid peroxide radicals.

\section{Reactive species in the human body}

Despite the non-reactivity of triplet oxygen, the human body is capable to use triplet oxygen for essential reactions. The enzymes that can bind triplet oxygen mostly contain a haem moiety with iron as the active metal ion. The transition metals (like iron) have several excited states with only small differences in energy levels. These small differences facilitate easy changes between the different electronic states (singlet, triplet, quintet and septet), which makes it possible for molecular oxygen (both singlet and triplet) to bind to the iron metal in the haem. Due to spin-orbit coupling of the haem and the oxygen, spin inversion is facilitated and a singlet complex $\mathrm{Fe}_{-} \mathrm{O}_{2}$ is formed [4]. The formation of this singlet complex is necessary to perform the essential reactions with other molecules that require molecular oxygen.

One important family of enzymes that use molecular oxygen is the NADPH oxidase family. Seven forms of this family are identified: five forms of NADPH oxidase (numbered as NOX till $\mathrm{NOX}_{5}$ ) and two dual oxidases (DUOX, and DUOX ${ }_{2}$ ).

The active oxidase of this family of enzymes consists of two transmembrane subunits that contain a haem group each $[9,10]$. All the family members have a binding site for the co-factors FAD and $\mathrm{NADPH}$. The NADPH in the cytosol supplies the electrons necessary to reduce molecular oxygen. Via FAD and the haem group, which has bound the oxygen molecule, the electrons reduce molecular oxygen to superoxide (one electron reduction by $\mathrm{NOX}_{1-3}$ and $\mathrm{NOX}_{5}$ ) or hydrogen peroxide (two electron reduction by $\mathrm{NOX}_{4}$, DUOX ${ }_{1}$ and $\left.\mathrm{DUOX}_{2}\right)[9,11]$. The reduced oxygen is released on the outside of the cell membrane.

The NOX enzymes are present in many different types of cells (e.g. epithelial cells, smooth muscle cells, fibroblasts, osteoclasts). The superoxide and hydrogen peroxide are generated in the close vicinity of those cells and could have different functions. In epithelial cells and inflammatory cells, these reactive species contribute to the inflammatory response by damaging the important structures of bacteria and viruses. In osteoclasts, superoxide contributes to bone resorption [12]. The produced reactive oxygen species also act as signaling molecules, which makes them important contributors to the maintenance of life [13-15].

Once hydrogen peroxide is formed, a second class of enzymes, peroxidases, can use this peroxide to produce more potent reactive species. Peroxidases, in general, use peroxides ( $\mathrm{R}-\mathrm{O}-\mathrm{O}-\mathrm{R}$ ', hydrogen peroxide or lipid peroxide) to produce hydroxyl groups (R-O-H) or hydroxyl radicals (from $\mathrm{H}_{2} \mathrm{O}_{2}$ ). 
The haem group of peroxidases differs slightly from the haem groups of other haem containing enzymes. The electronic states of the haem of peroxidases are frequently a doublet, a quartet or a sextet state, which will bind hydrogen peroxide (a singlet state) more easily than triplet oxygen [4]. One example of a peroxidase is myeloperoxidase (MPO). MPO is abundantly present in neutrophils where it catalyzes the main reaction of hydrogen peroxide and chlorine ions to hypochlorous acid [16]. This hypochlorous acid reacts easily with the proteins and cellular structures of bacteria and viruses or stimulates (indirectly) the inflammatory response.

A third family of haem containing enzymes are the nitric oxide synthases (NOS). These enzymes (three forms are identified in humans, eNOS, iNOS and nNOS) are capable to form the reactive species NO: Instead of reduction of molecular oxygen, these enzymes require arginine (or argininelike compounds) as a substrate to form NO: The amine group of L-arginine is oxidized by the molecular oxygen bound to the haem group and the intermediate hydroxylamine is formed. This hydroxylamine is further oxidized and citrulline and nitric oxide are formed [17]. The electrons necessary for this reaction are donated by NADPH.

Nitric oxide is a key molecule in many processes in the human body. It regulates vascular tone and blood flow, leukocyte adhesion and platelet aggregation, mitochondrial oxygen consumption, angiogenesis, neuronal survival and many other processes [18, 19]. This regulation can be performed directly by $\mathrm{NO}$; via formation of peroxynitrite or via the nitrosylation of specific proteins [20].

The families of enzymes described above are highly regulated in their main function of producing reactive oxygen species. However, not all haem containing proteins have a primary function of producing reactive oxygen species. The entire cytochrome P450 (CYP) family of enzymes contains a haem and their main function is the hydroxylation of compounds. The hydroxylation of the compound starts with most CYP-enzymes with the interaction of a substrate to a protein substrate binding site. This interaction changes the spin-state of the haem from low spin to high spin, which facilitates the binding of molecular oxygen. Once the $\mathrm{Fe}_{-} \mathrm{O}_{2}$ complex is reduced by the electrons donated by NADPH, the substrate is hydroxylated via several intermediates formed. For almost every CYP family member, this reaction is performed without the production of reactive species. However, some enzymes are prone to produce reactive oxygen species during their catalytic cycle, specifically the isoform CYP2E1.

The haem of CYP2E1 is, in contrast to other CYP-enzymes, frequently in the high spin state independently of the presence of a substrate [21,22]. Molecular oxygen will bind easily to the haem and will be reduced without the presence of a substrate to form a hydroxylated product (known as uncoupling, oxidase or futile cycling). This reduction of molecular oxygen greatly resembles the catalytic cycle of the nitric oxide synthases, which also have their haem group in the high spin state [23]. The partially reduced oxygen, as superoxide or hydrogen peroxide [24], could easily dissociate from the active site of the enzyme. The production of reactive oxygen species can also be due to the stability of the $\mathrm{Fe}_{-} \mathrm{O}_{2}$ complex. The small differences between the energy levels that make it possible 
for molecular oxygen to bind to the haem group, are also the cause of the instability of the $\mathrm{Fe}-\mathrm{O}_{2}$ complex. If the reactions in the catalytic cycle of cytochrome $\mathrm{P} 450$ are too slow, the $\mathrm{Fe}-\mathrm{O}_{2}$ complex will dissociate and superoxide, hydrogen peroxide or molecular oxygen will leave the active site of the enzyme [23].

These reactive oxygen species, released in the interior of the cell, damage the mitochondria, DNA and cellular membranes, which can cause irreversible damage to cells and trigger constantly the inflammation response [3].

Patients with non-alcoholic steatohepatitis (NASH) have high levels of CYP2E1 [25-28]. The high production of reactive oxygen species by CYP2E1 results in mitochondrial dysfunction, lipid peroxidation, induction of several cytokines and necrosis. This damage to the hepatocytes triggers the inflammation response [29] and the liver of these patients could become fibrotic and cirrhotic [30].

Other diseases are also associated with increased levels of reactive oxygen species, among them are cancer [31], chronic obstructive pulmonary disease [32], and diabetes [33].

\section{Antioxidants in human body}

The damage caused by the undesired production of reactive oxygen species can be prevented by antioxidants. An antioxidant is defined as any substance that when present at low concentrations, compared to those of an oxidizable substrate significantly delays or prevents oxidation of that substrate $[5,34,35]$. The oxidation of the substrates (proteins, lipid membrane molecules or DNA) decreases the cellular viability and therefore has to be prevented. Reactive species have different chemical properties that determine their reactivity, and antioxidants have to adapt to this by offering protection via different mechanisms.

The reactivity of singlet oxygen is based on the configuration of the electrons, and carotenoids are excellent protectors of singlet oxygen damage [36]. Carotenoids contain a long central chain of conjugated double bonds, indicating that the molecule has many possible excited states. The differences between the energy levels of these excited states are small, which is crucial for the quenching of singlet oxygen. The energy levels of singlet oxygen and triplet oxygen differ $0.98 \mathrm{eV}$. Since an excited triplet state of the carotenoid differs in the same order of magnitude from its singlet ground state, the energy of singlet oxygen can easily be transferred to the carotenoid via intersystem crossing. As a result, oxygen returns to its stable triplet state and the carotenoid is in its excited triplet state. This triplet state of the carotenoid can however easily and harmlessly decay to its stable singlet ground state, due to the extensive conjugated system [36-38]. The energy of this decay from triplet to singlet state is released via phosphorescence. Since the carotenoid does not change chemically when it quenches singlet oxygen, it can be considered as a catalytic antioxidant.

In addition to the quenching ability of carotenoids, singlet oxygen can also react with the double bounds of the carotenoids, resulting in an endoperoxide. In this way, the carotenoids act as sacrificial antioxidants. However, chemical reactions of singlet oxygen with carotenoids contribute for only 0.05 percent to the protection of the cellular target [37]. 
The protection of antioxidants against radical damage has to be via a different mechanism. The reactivity of radicals is based on the presence of an unpaired electron. To decrease the reactivity, unpaired electrons have to combine and an electron pair has to be formed (with the exception of triplet oxygen). This is achieved by the reaction of two radicals. However, this reaction is not included in the definition of an antioxidant, since two reactive species with an unpaired electron are involved. To neutralize a radical, an electron can be donated by a compound, i.e. the antioxidant, to the radical. The antioxidant itself now becomes a radical. This newly formed antioxidant radical is more stable than the scavenged radical. This stability is mostly due to delocalization of the unpaired electron, which decreases the reactivity of the compound. This mechanism is seen e.g. by the antioxidants quercetin, a-tocopherol and carotenoids when they react with radicals [37, 38].

The reactivity of a radical can also be terminated by donating a hydrogen atom $\left(H^{*}\right)$ to the radical [39]. The electron then forms a 1s bound with the hydrogen. Phenolic compounds can easily donate a hydrogen atom to the radical.

The above described mechanisms of scavenging of radicals are independent of the type of radical; the primary produced radicals ( $\mathrm{OH}, \mathrm{O}_{2}{ }^{-}, \mathrm{NO} \cdot$ ) or derived radicals (lipid radical, lipid peroxides, tyrosine radicals). The activity of an antioxidant however does depend on the type of radical. Carotenoids are excellent scavengers of secondary radicals, the so-called chain breaking antioxidants, although they are poor scavengers of other radicals [38].

The protection against non-radical reactive species $\left({ }^{1} \mathrm{O}_{2}, \mathrm{HOCl}, \mathrm{H}_{2} \mathrm{O}_{2}\right.$, peroxynitrite) has to be performed by reacting with these reactive species before they can react with the cellular target.

Besides the antioxidant compounds, the human body also contains enzymes that have antioxidant activity. Superoxide dismutase, of which isoenzymes are present in the mitochondria, the cytosol and extracellular, catalyzes the dismutation of superoxide to hydrogen peroxide and triplet oxygen. The enzyme catalase transforms hydrogen peroxide into molecular oxygen and water [34]. Glutathione peroxidase detoxifies hydrogen peroxide using the reductive power of glutathione (GSH) that is oxidized to the disulfide form (GSSG). GSSG can be regenerated by GSSG reductase to GSH.

The protection against oxidative damage offered by antioxidants discussed above is performed once the reactive oxygen species have already been produced. However, the definition of an antioxidant also includes the protection by prevention of generation of the reactive species. This prevention of generation can be obtained via inhibition of the enzymes that produce reactive species. Inhibitors of CYP2E1 can therefore be considered as antioxidants. In addition, the scavenging of the precursors (e.g. nitrate) of the reactive species or inactivation of mediators (transition metals) inhibits the generation of radicals.

\section{Aim of the thesis}

Since antioxidants can act via different mechanisms, it is difficult to determine the aspects that qualify an antioxidant as a good antioxidant. The activity of antioxidants is mostly determined in competition assays. The conditions and limitations of these assays to determine the antioxidant 
activity are not acknowledged (chapters 2, 3 and 4).

In biological systems, the activity of antioxidants becomes more complex. Not only competition between antioxidants and biological target molecules is important in determining the actual activity of antioxidants. The antioxidant function of compounds also includes the inhibition of radical generating enzymes (chapters 5 and 6 ).

In vivo, the situation becomes even more complex, since antioxidants cooperate in an intricate network. The antioxidant effect of mixtures (e.g. derived from a food matrix) might be expressed as a general antioxidant status (chapter 7).

The aim of this thesis is to provide insight into the correct way to measure scavenging antioxidant activity. Moreover, it is illustrated how inhibition of enzymatic oxidative activity might have clinical use in pharmacological treatment of diseases. Finally, it is shown that changes in antioxidant status can be obtained by relatively simple dietary interventions. The overall aim is to study the activity of antioxidants from model to man. 


\section{References}

1. Frank, L. and Massaro, D., Oxygen toxicity. Am. J. Med., 1980. 69(1): p. 117-126.

2. Matchett, G.A., Martin, R.D., and Zhang, J.H., Hyperbaric oxygen therapy and cerebral ischemia: neuroprotective mechanisms. Neurol. Res., 2009. 31(2): p. 114-121.

3. Davies, M.J., The oxidative environment and protein damage. Biochim. Biophys. Acta, 2005. 1703(2): p. 93109.

4. Jensen, K.P. and Ryde, U., How $\mathrm{O} 2$ binds to heme: reasons for rapid binding and spin inversion. J. Biol. Chem. 2004. 279(15): p. 14561-14569.

5. Halliwell, B. and Gutteridge, J.M.C., Free radicals in biology and medicine. 3rd ed. 1999, Oxford: Oxford University Press. 936.

6. Armesto, X.L., et al., First Steps in the Oxidation of Sulfur-Containing Amino Acids by Hypohalogenation:Very Fast Generation of Intermediate Sulfenyl Halides and Halosulfonium Cations. Tetrahedron, 2000. 56(8): p. 1103-1109.

7. Nagy, P. and Ashby, M.T., Reactive sulfur species: kinetics and mechanisms of the oxidation of cysteine by hypohalous acid to give cysteine sulfenic acid. J. Am. Chem. Soc., 2007. 129(45): p. 14082-14091.

8. Ferrer-Sueta, G. and Radi, R., Chemical biology of peroxynitrite: kinetics, diffusion, and radicals. ACS Chem Biol, 2009. 4(3): p. 161-177.

9. van der Vliet, A., NADPH oxidases in lung biology and pathology: host defense enzymes, and more. Free Radic. Biol. Med., 2008. 44(6): p. 938-955.

10. Lambeth, J.D., Kawahara, T., and Diebold, B., Regulation of Nox and Duox enzymatic activity and expression. Free Radic. Biol. Med., 2007. 43(3): p. 319-331.

11. Shatwell, K.P. and Segal, A.W., NADPH oxidase. Int. J. Biochem. Cell Biol., 1996. 28(11): p. 1191-1195.

12. Banfi, G., Iorio, E.L., and Corsi, M.M., Oxidative stress, free radicals and bone remodeling. Clin. Chem. Lab. Med., 2008. 46(11): p. 1550-1555.

13. Bartosz, G., Reactive oxygen species: destroyers or messengers? Biochem. Pharmacol., 2009. 77(8): p. 13031315.

14. D'Autreaux, B. and Toledano, M.B., ROS as signalling molecules: mechanisms that generate specificity in ROS homeostasis. Nat Rev Mol Cell Biol, 2007. 8(10): p. 813-824.

15. Droge, W., Free radicals in the physiological control of cell function. Physiol. Rev., 2002. 82(1): p. 47-95.

16. Malle, E., et al., Myeloperoxidase: a target for new drug development? Br. J. Pharmacol., 2007. 152(6): p. 838854.

17. Nishida, C.R., et al., Electron supply and catalytic oxidation of nitrogen by cytochrome P450 and nitric oxide synthase. Drug Metab. Rev., 2002. 34(3): p. 479-501.

18. Ferdinandy, P., Peroxynitrite: just an oxidative/nitrosative stressor or a physiological regulator as well? Br. J. Pharmacol., 2006. 148(1): p. 1-3.

19. Luiking, Y.C., Engelen, M.P., and Deutz, N.E., Regulation of nitric oxide production in health and disease. Curr. Opin. Clin. Nutr. Metab. Care, 2009.

20. Benhar, M., Forrester, M.T., and Stamler, J.S., Protein denitrosylation: enzymatic mechanisms and cellular functions. Nat Rev Mol Cell Biol, 2009. 10(10): p. 721-732.

21. Guengerich, F.P. and Johnson, W.W., Kinetics of ferric cytochrome P450 reduction by NADPH-cytochrome P450 reductase: rapid reduction in the absence of substrate and variations among cytochrome P450 systems. Biochemistry (Mosc). 1997. 36(48): p. 14741-14750.

22. Zhukov, A. and Ingelman-Sundberg, M., Relationship between cytochrome P450 catalytic cycling and stability: fast degradation of ethanol-inducible cytochrome P450 2E1 (CYP2E1) in hepatoma cells is abolished by inactivation of its electron donor NADPH-cytochrome P450 reductase. Biochem. J., 1999. 340 ( Pt 2): p. 453-458.

23. Gorren, A.C. and Mayer, B., Nitric-oxide synthase: a cytochrome P450 family foster child. Biochim. Biophys. Acta, 2007. 1770(3): p. 432-445. 
24. Zangar, R.C., Davydov, D.R., and Verma, S., Mechanisms that regulate production of reactive oxygen species by cytochrome P450. Toxicol. Appl. Pharmacol., 2004. 199(3): p. 316-331.

25. Lieber, C.S., CYP2E1: from ASH to NASH. Hepatol Res, 2004. 28(1): p. 1-11.

26. Robertson, G., Leclercq, I., and Farrell, G.C., Nonalcoholic steatosis and steatohepatitis. II. Cytochrome P-450 enzymes and oxidative stress. Am J Physiol Gastrointest Liver Physiol, 2001. 281(5): p. G1135-1139.

27. Chtioui, $H_{\text {., }}$ et al., Expression and activity of the cytochrome P450 $2 \mathrm{E} 1$ in patients with nonalcoholic steatosis and steatohepatitis. Liver Int, 2007. 27(6): p. 764-771.

28. Weltman, M.D., et al., Hepatic cytochrome P450 2E1 is increased in patients with nonalcoholic steatohepatitis. Hepatology, 1998. 27(1): p. 128-133.

29. Rensen, S.S., et al., Increased hepatic myeloperoxidase activity in obese subjects with nonalcoholic steatohepatitis. Am. J. Pathol., 2009. 175(4): p. 1473-1482.

30. Te Sligte, K., et al., Non-alcoholic steatohepatitis: review of a growing medical problem. Eur J Intern Med, 2004. 15(1): p. 10-21.

31. Valko, M., et al., Free radicals, metals and antioxidants in oxidative stress-induced cancer. Chem. Biol. Interact., 2006. 160(1): p. 1-40.

32. Rahman, I., Antioxidant therapeutic advances in COPD. Ther Adv Respir Dis, 2008. 2(6): p. 351-374.

33. Mehta, J.L., et al., Oxidative stress in diabetes: a mechanistic overview of its effects on atherogenesis and myocardial dysfunction. Int. J. Biochem. Cell Biol., 2006. 38(5-6): p. 794-803.

34. Aruoma, O.I., Methodological considerations for characterizing potential antioxidant actions of bioactive components in plant foods. Mutat. Res., 2003. 523-524: p. 9-20.

35. Magalhaes, L.M., et al., Methodological aspects about in vitro evaluation of antioxidant properties. Anal. Chim. Acta, 2008. 613(1): p. 1-19.

36. Di Mascio, P., Kaiser, S., and Sies, H., Lycopene as the most efficient biological carotenoid singlet oxygen quencher. Arch. Biochem. Biophys., 1989. 274(2): p. 532-538.

37. Stahl, W. and Sies, H., Antioxidant activity of carotenoids. Mol. Aspects Med., 2003. 24(6): p. 345-351.

38. Edge, R., McGarvey, D.J., and Truscott, T.G., The carotenoids as anti-oxidants--a review. J. Photochem. Photobiol. B., 1997. 41(3): p. 189-200.

39. Prior, R.L., Wu, X., and Schaich, K., Standardized methods for the determination of antioxidant capacity and phenolics in foods and dietary supplements. J. Agric. Food Chem., 2005. 53(10): p. 4290-4302. 
$d x_{1}=-k_{j} x_{1}$
$d x_{2}=k_{2} x_{1}$ 


\section{Chapter 2}

Evaluation of the accuracy of antioxidant competition assays Incorrect assumptions with major impact

Jiska M.Balk, Aalt Bast, Guido R.M.M.Haenen,

Free Radical Biology and Medicine, Volume 47, pages: 135-144, 2009 


\section{Abstract}

The activity of antioxidants is frequently determined in competition assays. In these assays an antioxidant (A) and detector molecule (D) compete for the reactive species (R). The competitive inhibitory effect of $A$ on the reaction of $D$ with $R$ is a measure of the antioxidant activity of $A$. In determining the activity of $A$, it is in general incorrectly assumed that the concentrations of $A$ and $D$ remain equal to the initial concentration. However, the principle of the assay is that some $A$ and $D$ is consumed and consequently the concentration of $A$ and $D$ will decrease during a competition assay, resulting in a deviation in the observed antioxidant activity. Computer modeling was used to obtain a graphical tool to estimate the extent of the deviation caused by the incorrect assumption that the concentrations of A and D do not decrease. Several competition assays were evaluated using this graphical tool, demonstrating that frequently inaccurate antioxidant activities have been reported. In general, differences between antioxidants are underestimated and the activity of all antioxidants shifts toward the antioxidant activity of $\mathrm{D}$. A strategy is provided to improve the accuracy of a competition assay. To obtain accurate results in a competition assay, the reaction rate constant of the detector molecule with the reactive species should be comparable to that of the antioxidant. In addition, the concentration of reactive species should be as low as possible. 


\section{Introduction}

Oxidative species play a pivotal role in numerous patho-physiological processes ranging from cancer to toxic effects of drugs [1]. This involvement of oxidative reactive species (including radicals and non-radicals such as hypochlorous acid and peroxynitrite) forms the rationale for antioxidant research. The scavenging activity of an antioxidant is the first line of defense against these reactive species. In addition to directly scavenging the initial reactive species, antioxidants can protect by their chain breaking activity. In these types of protection, antioxidants react with the reactive species. The rate of the reaction of the antioxidant with the reactive species determines the effectiveness of the antioxidant.

Direct measurement of the reaction rate of the radical scavenging reaction by the antioxidant requires expensive and sophisticated equipment, such as pulse radiolysis and stopped flow. In the case of highly reactive species, the current instruments still fall short. Alternatively, the reaction rate of an antioxidant with the reactive species $\left(v_{a}\right)$ can be measured in an indirect way in a relatively simple competition assay.

In these widely used competition assays, a reactive species (e.g. radicals, hypochlorous acid, peroxynitrite) is added to a mixture of a fixed concentration of a detector compound and a variable concentration of an antioxidant. The antioxidant (A) and detector (D) compete for the reactive species (R) as outlined in figure 1 . The ability of the antioxidant to protect the detector is determined by the reaction rate of the antioxidant with the reactive species. The efficacy of the antioxidant is generally described by the $\mathrm{EC}_{50}$ value, which is used to rank antioxidants. The $\mathrm{EC}_{50}$ is the concentration of antioxidant that protects the detector by 50 percent, compared to the situation in which no antioxidant has been added to the system. Values of the $\mathrm{EC}_{50}$ that are lower than the concentration of the detector indicate that the antioxidant reacts faster with the reactive species than the detector does. $\mathrm{A} \mathrm{low}_{\mathrm{EC}}$ indicates a high antioxidant efficacy.

The detector is a compound that reacts readily with the reactive species, preferably with a known reaction rate constant. The reaction product formed out of the detector is commonly used to determine the $\mathrm{EC}_{50^{\prime}}$ although other strategies, e.g. measuring the consumption of the detector molecule, are also used.

Numerous competition assays have been designed. The use of various detector molecules, pH values, solvents and temperatures makes it hard to compare the results obtained from these assays. The reaction rate constant of a particular antioxidant obtained in different assays may differ by several orders of magnitude [2].

The cause of this difference is not fully understood, although it has to originate from the experimental design and the assumptions made in evaluating the activity. In this study, the basic premises in a competition assay are analyzed in detail (e.g. with computer modeling) to identify critical factors in the experimental procedure of a competition assay. 


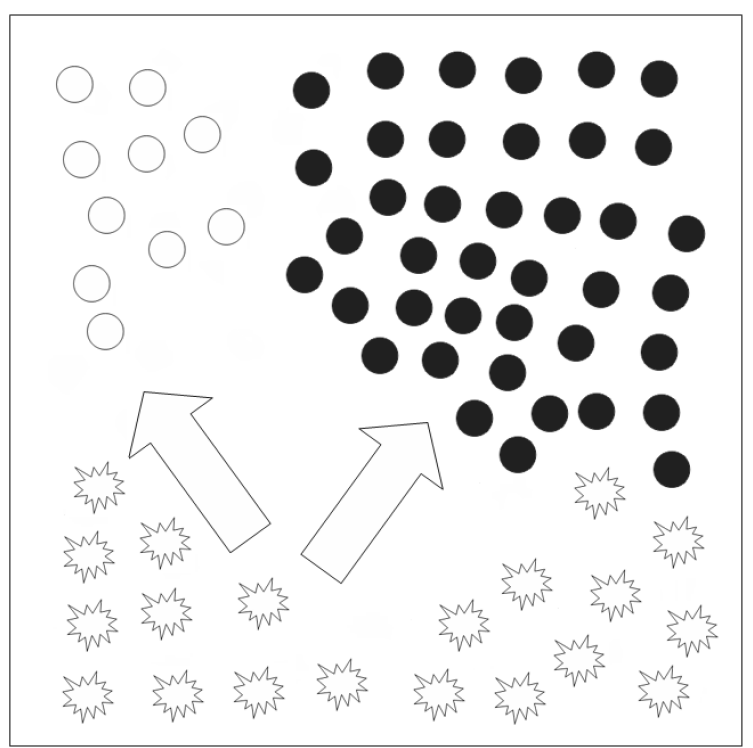

Figure 1. Schematic representation of a competition assay. The detector (depicted as closed circles) competes with the antioxidant (depicted as open circles) for the reactive species (depicted as stars). The arrow points to the molecule of reaction (antioxidant or detector). The width of the arrow represents the reaction rate $\left(v_{a}\right.$ or $\left.v_{d}\right)$. It is often overlooked that, during a competition assay, the reaction rate of the reactive species with the antioxidant or the detector is not constant. As emphasized in this study, both the antioxidant and the detector are consumed during a competition assay and this affects the reaction rates. This can result in a considerable error in the reported activity of the antioxidant.

\section{Method}

A competition assay consists of three compounds, the detector (D), the antioxidant (A) and the reactive species (R). R reacts with either $\mathrm{D}$ or $\mathrm{A}$ (equations 1 and 2 ).

$D+R \stackrel{k_{d}}{\longrightarrow} P D$

Equation 1

$A+R \stackrel{k_{a}}{\longrightarrow} P A$

Equation 2

$P D$ and PA are the products formed from the reaction of $R$ with $D$ and $R$ with $A$, respectively. The $k_{d}$ and the $k_{a}$ are the second order reaction rate constants. It is assumed that additional reactions between the various compounds do not occur. Both reactions 1 and 2 were described as second order kinetic reactions with a stoichiometry of one (equations 3-7).

$v_{r}=-\frac{d[R]}{d t}=+k_{d}[D] \cdot[R]+k_{a}[A] \cdot[R]$

Equation 3

$v_{d}=-\frac{d[D]}{d t}=+k_{d}[D] \cdot[R]$

Equation 4 
$v_{a}=-\frac{d[A]}{d t}=+k_{a}[A] \cdot[R]$

$v_{p d}=+\frac{d[P D]}{d t}=-k_{d}[D] \cdot[R]$

$v_{p a}=+\frac{d[P A]}{d t}=-k_{a}[A] \cdot[R]$
Equation 5

Equation 6

Equation 7

$\checkmark$ represents the reaction rate of compounds as specified in subscript.

This system of differential equations was solved numerically by Matlab (version R2007a, The MathWorks, Inc. Natick, Massachusetts, USA) with a built-in function. The initial concentrations ([D] $]_{0^{\prime}}$ $[A]_{0}$ and $\left.[R]_{0}\right)$ and the second order rate constants $\left(k_{a}\right.$ and $\left.k_{d}\right)$ were defined by input. In all simulations, the $[D]_{0}$ and $k_{d}$ were kept constant at $1 \mathrm{mM}$ and $1 \mathrm{mM}^{-1} \mathrm{~s}^{-1}$, respectively. The concentration of each compound was calculated for each time step (figure 2). The reactions were considered to be completed when [R] had reached a concentration of less than 0.001 percent of its initial concentration.

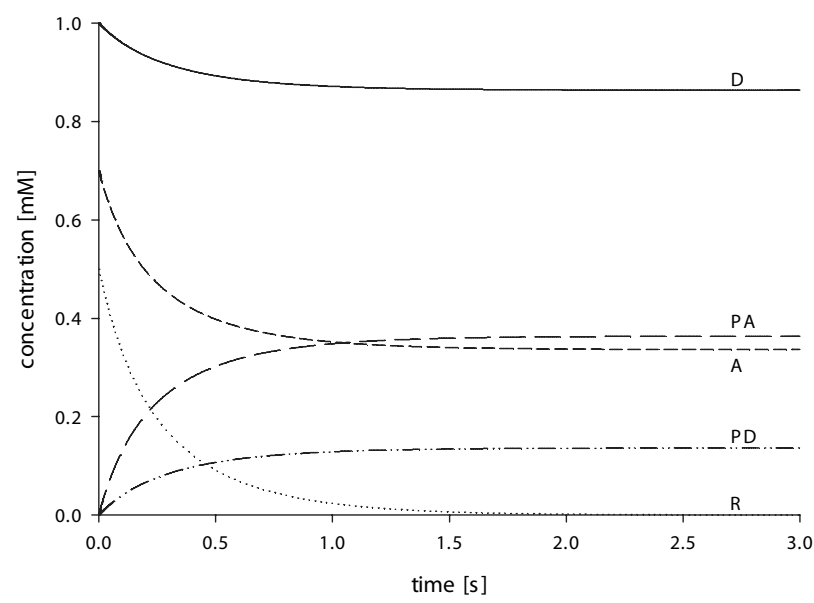

Figure 2. Simulation of the change in concentration of reactants and products with time for a simple competition experiment. An antioxidant (A) and detector (D) compete for the radical (R). The concentrations of the products formed from $A(P A)$ and $D(P D)$ in the second order reactions are also depicted. The initial concentrations are $[D]_{0}=1 \mathrm{mM}$, $[A]_{0}=0.7 \mathrm{mM}$ and $[R]_{0}=0.5 \mathrm{mM}$. The second order reaction rate constants used are $k_{d}=1 \mathrm{mM}^{-1} \mathrm{~s}^{-1}$ and $\mathrm{k}_{a}=5 \mathrm{mM}^{-1} \mathrm{~s}^{-1}$ and other reactions do not take place in the system. It is assumed that the reactions have reached completion when $[R]$ is less than $0.001 \%$ of its initial concentration, which is the case for the depicted simulation. 
The system of differential equations was solved for several initial concentrations of $[A]_{0}$ to obtain an $\mathrm{EC}_{50}$ of the antioxidant. The $\mathrm{EC}_{50}$ is defined as the initial concentration of antioxidant $\left([\mathrm{A}]_{0}\right)$ that reduces the amount of formed product PD of the detector (D) with reactive species (R) by 50 percent, compared to the situation in which no antioxidant has been added to the reaction system. Although $[A],[D],[P A]$ and $[P D]$ can all be used to determine the $\mathrm{EC}_{50}$ (and every approach will give the same result), we have used the final concentration of $\mathrm{PD}$ formed to determine the $\mathrm{EC}_{50^{\prime}}$, because this is commonly used and from an analytical point of view it will give the most accurate results in practice. The initial concentration of $[A]_{0}$ was increased in steps of $0.01 \mathrm{mM}$ and the other initial values $\left(k_{d^{\prime}}\right.$ $\mathrm{k}_{\mathrm{a}^{\prime}}[\mathrm{D}]_{0}$ and $[\mathrm{R}]_{0}$ ) were not altered. The $\mathrm{EC}_{50}$ was calculated using linear interpolation of $[\mathrm{A}]_{0}$. Various antioxidants, i.e. various values of $k_{a^{\prime}}$ were investigated.

The $\mathrm{EC}_{50}$ was determined for several $[\mathrm{R}]_{0}$ (from $0.01 \mathrm{mM}$ to $1 \mathrm{mM}$ with a step size of $0.01 \mathrm{mM}$ ) to investigate the influence of $[R]_{0}$ on the $E C_{50}$. The $E C_{50}$ determined in this way is referred to as the observed $\mathbf{E C}_{\mathbf{5 0}}$. The observed $\mathrm{EC}_{50}$ is derived from computer simulation or experimental determination of the $\mathrm{EC}_{50}$.

The simulation was extended to evaluate the contribution of the oxidation product of $A(P A)$ on the observed $\mathrm{EC}_{50}$ (equation 8).

$P A+R \stackrel{k_{a 2}}{\longrightarrow} P P A$

Equation 8

In competition assays, $\mathrm{k}_{\mathrm{a}}$ is commonly calculated using the $\mathrm{EC}_{50^{\prime}}$ according to equation 9 [3-5].

$k_{a}=k_{d} \cdot \frac{[D]_{0}}{E C_{50}}$

Equation 9

This equation was derived from equations 4 and 5 , as is shown by the following equations. In this derivation, it was assumed that $[D]$ is $[D]_{0}$ and $[A]$ is $[A]_{0}$ throughout the competition experiment. This assumption is usually made, although the present evaluation will show that it is a fundamental error. At the $E C_{50^{\prime}}$ the reaction rate of $A$ with $R$ equals that of $D$ with $R$, and therefore:

$$
\frac{d[D]}{d t}=\frac{d[A]}{d t}
$$

Equation 10

At the $\mathrm{EC}_{50}\left([\mathrm{~A}]_{0}=\mathrm{EC}_{50}\right)$, equation 11 can be derived.

$$
\begin{array}{ll}
k_{d} \cdot[D]_{0} \cdot[R]_{0}=k_{a} \cdot E C_{50} \cdot[R]_{0} & \text { Equation } 11 \\
E C_{50}=\frac{k_{d}}{k_{a}} \cdot[D]_{0} & \text { Equation } 12
\end{array}
$$

In our model, $\mathrm{k}_{\mathrm{a}^{\prime}} \mathrm{k}_{\mathrm{d}}$ and $[\mathrm{D}]_{0}$ are defined as input parameters. Therefore, the $\mathrm{EC}_{50}$ can be calculated according to equation 12. The $\mathrm{EC}_{50}$ calculated in this way is referred to as the ideal $\mathbf{E C}_{\mathbf{5 0}}$. In a given antioxidant assay, the ideal $\mathrm{EC}_{50}$ of antioxidants is directly linked to $\mathrm{k}_{\mathrm{a}}$ (see equations 9 and 12). 


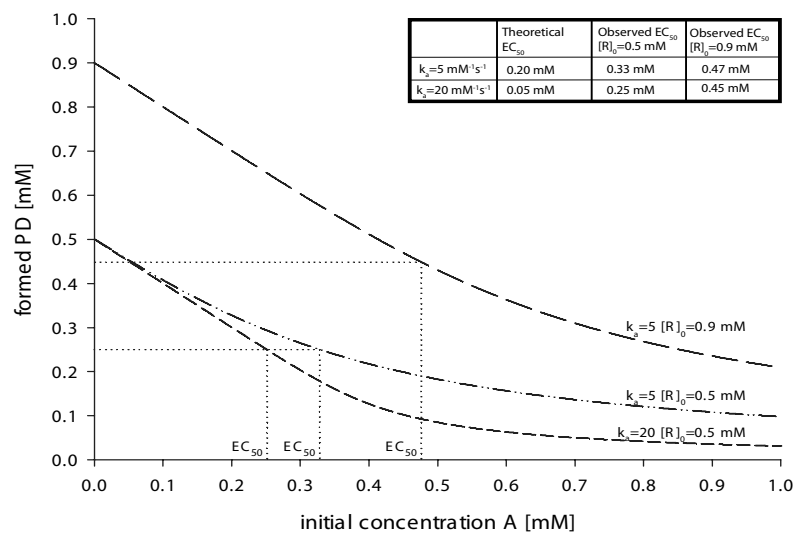

Figure 3. Simulation of the effect of the initial concentration of radical $(R)$ and the second order rate constant of the antioxidant ( $k_{d}$ on the observed activity of the antioxidant. The activity is determined by the $E C_{50}$ that is obtained (the observed $E C_{50}$ by varying $[A]_{0}$. The observed $E C_{50}$ is the $[A]_{0}$ that reduces the formation of $P D$ by $50 \%$. In all simulations $[D]_{0}=1 \mathrm{mM}$ and $\mathrm{k}_{d}=1 \mathrm{mM}^{-1} \mathrm{~s}^{-1}$. The values of $\mathrm{k}_{a}$ (either 5 or $20 \mathrm{mM}^{-1} \mathrm{~s}^{-1}$ ) and $[R]_{0}$ (either $0.5 \mathrm{mM}$ or $0.9 \mathrm{mM}$ ) are indicated. Under the conditions used in the figure, the effect of increasing $[R]_{0}$ from $0.5 \mathrm{mM}$ to $0.9 \mathrm{mM}$ has a higher impact on the observed activity of $A$, expressed in the $E C_{50}$ than increasing the $k_{a}$ from 5 to $20 \mathrm{mM}^{-1} \mathrm{~s}^{-1}$.

\section{Results}

Figure 2 shows a typical example of the simulation of a single experiment in a competition assay. In the simulation (figure 3), an antioxidant with $\mathrm{k}_{\mathrm{a}}=5 \mathrm{mM}^{-1} \mathrm{~s}^{-1}$ was compared with an antioxidant with $\mathrm{k}_{\mathrm{a}}=20 \mathrm{mM}^{-1} \mathrm{~s}^{-1}$. The latter is four times more effective. Therefore, the ideal $\mathrm{EC}_{50}$ of the slower reacting antioxidant $\left(\mathrm{k}_{\mathrm{a}}=5 \mathrm{mM}^{-1} \mathrm{~s}^{-1}\right.$, ideal $\left.\mathrm{EC}_{50}=0.2 \mathrm{mM}\right)$ is four times the ideal $\mathrm{EC}_{50}$ of the fast reacting antioxidant $\left(\mathrm{k}_{\mathrm{a}}=20 \mathrm{mM}^{-1} \mathrm{~s}^{-1}\right.$, ideal $\left.\mathrm{EC}_{50}=0.05 \mathrm{mM}\right)$. In the simulation depicted in figure 3 , it was found that the observed $\mathrm{EC}_{50}$ 's are $0.32 \mathrm{mM}$ and $0.25 \mathrm{mM}$ if $[\mathrm{R}]_{0}=0.5 \mathrm{mM}$, indicating that the observed $\mathrm{EC}_{50}$ 's of both antioxidants are quite similar, in contrast to the expected ideal $\mathrm{EC}_{50}$. This demonstrates that under the conditions used, the observed $\mathrm{EC}_{50}$ is hardly influenced by the $\mathrm{k}_{\mathrm{a}}$. Increasing $[\mathrm{R}]_{0}$ from $0.5 \mathrm{mM}$ to $0.9 \mathrm{mM}$ changes the observed $\mathrm{EC}_{50}$ of the slow-reacting antioxidant $\left(\mathrm{k}_{\mathrm{a}}=5 \mathrm{mM}^{-1} \mathrm{~s}^{-1}\right)$ from $0.32 \mathrm{mM}$ to $0.48 \mathrm{mM}$. This indicates that under the conditions used, the influence of variation of $[R]_{0}$ (i.e. changing $[R]_{0}$ from $0.5 \mathrm{mM}$ to $0.9 \mathrm{mM}$ ) on the observed $\mathrm{EC}_{50}$ is larger than that of changing the $k_{a}$ (i.e. changing $k_{a}$ from $5 \mathrm{mM}^{-1} \mathrm{~s}^{-1}$ to $20 \mathrm{mM}^{-1} \mathrm{~s}^{-1}$ ). It is generally assumed that the $\mathrm{EC}_{50}$ is independent of $[\mathrm{R}]_{0}$. The ideal $\mathrm{EC}_{50}$ is indeed independent of $[\mathrm{R}]_{0}$ (equation 12), but the observed $E C_{50}$ is greatly affected by $[R]_{0}$.

The influence of $[\mathrm{R}]_{0}$ on the observed $\mathrm{EC}_{50}$ was investigated in more detail (with the conditions used:

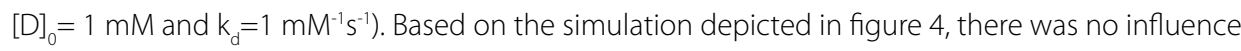
of $[\mathrm{R}]_{0}$ on the observed $\mathrm{EC}_{50}$ if the reaction rate constant of the antioxidant $\left(\mathrm{k}_{\mathrm{a}}\right)$ equals that of the detector $\left(k_{d}\right)$ (i.e., $k_{d}=k_{a}=1 m^{-1} s^{-1}$ ). When $k_{a}$ was smaller than $k_{d^{\prime}}$ the observed $E_{50}$ decreased at increasing $[R]_{0}$. Conversely, when $k_{a}$ was higher than $k_{d^{\prime}}$ the observed $E C_{50}$ increased at increasing $[\mathrm{R}]_{0}$. 


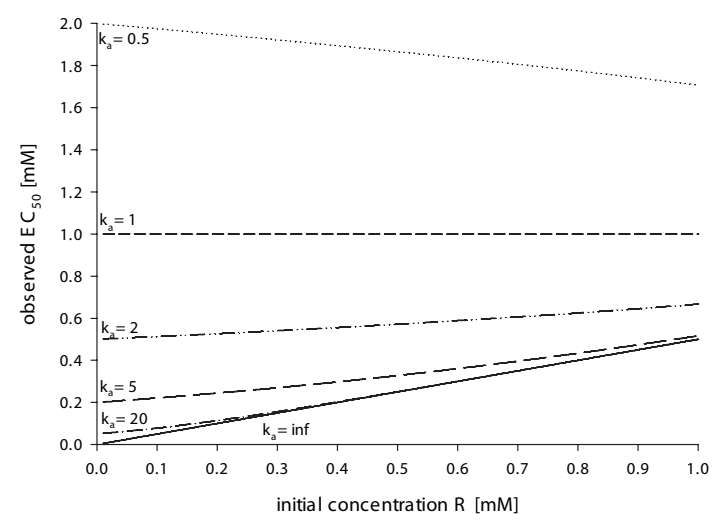

Figure 4. Simulation of the effect of the initial radical concentration on the observed activity. The observed $E C_{50}$ is determined at increasing $[R]_{0}$ for several antioxidants ( $k_{a}$ between 0.5 and $20 \mathrm{mM}^{-1} \mathrm{~s}^{-1}$ ). In all simulations the $[D]_{0}=1 \mathrm{mM}$ and the $k_{d}=1 \mathrm{mM}^{-1} \mathrm{~s}^{-1}$. If the $k_{a}$ is equal to the $k_{d}$ the $E C_{50}$ is independent of the concentration of $R$. If the $k_{a}$ is smaller than the $k_{d^{\prime}}$ the $E C_{50}$ decreases with increasing concentration of $R$. If the $k_{a}$ is higher than the $k_{d^{\prime}}$ the $E C_{50}$ increases with increasing concentration of $R$. The observed activity of those antioxidants reaches an asymptote at $E C_{50}=0.5^{*}[R]_{0}$ ( $k_{a}=$ infinite). Overall, the antioxidant activity levels off at increasing $[R]_{0}$ and the deviation of the observed $E C_{50}$ is toward $[D]_{0}$.

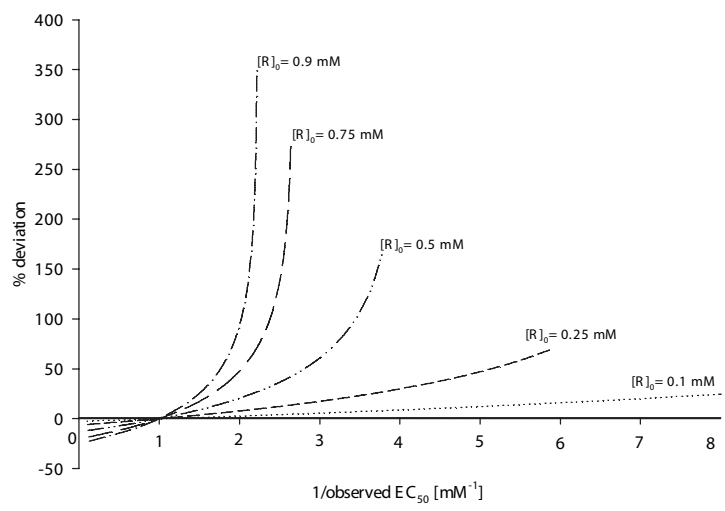

Figure 5. The deviation of the observed $E C_{50}$ from the ideal $E C_{50}$ at various concentrations of reactive species. The data is obtained from figure 4 with a $k_{a}$ from $0.1 \mathrm{mM}^{-1} \mathrm{~s}^{-1}$ to $10 \mathrm{mM}^{-1} \mathrm{~s}^{-1}$ and $\mathrm{k}_{d}$ is $1 \mathrm{mM}^{-1} \mathrm{~s}^{-1}$ and $[D]_{0}$ is $1 \mathrm{mM}$. The ideal $E C_{50}$ is calculated from equation 12. The deviation is calculated according to the equation: ((observed $E C_{50}-i_{d e a l} E C_{50}$ )/ideal $\left.E C_{50}\right) * 100 \%$. A negative deviation indicates an overestimation of the antioxidant activity (higher observed $E C_{50}$ relative to the ideal $E C_{50}$. A positive deviation indicates an underestimation of the antioxidant activity.

The simulation also reveals that if $\mathrm{k}_{\mathrm{a}}$ is much higher than $\mathrm{k}_{\mathrm{d}^{\prime}}$ the observed $\mathrm{EC}_{50}$ is independent of $k_{a}$ and $k_{d}$. The line of $k_{a}=20 \mathrm{mM}^{-1} \mathrm{~s}^{-1}$ is quite similar to the asymptote of $k_{a}=$ infinite. Under these conditions, the observed $\mathrm{EC}_{50}$ depends solely on $[\mathrm{R}]_{0^{\prime}}$, namely $\mathrm{EC}_{50}=0.5 \cdot[\mathrm{R}]_{0}$. It should be noted that the ideal $\mathrm{EC}_{50}$ depends linearly on $\mathrm{k}_{\mathrm{a}}$ and is independent of $[\mathrm{R}]_{0}$ (equation 12).

The observed $\mathrm{EC}_{50}$ is always higher than the ideal $\mathrm{EC}_{50}$ if $\mathrm{k}_{\mathrm{a}}$ is larger than $\mathrm{k}_{\mathrm{d}}$. As shown in figure 3 
(inset), this difference between the observed and the ideal $\mathrm{EC}_{50}$ increases upon increasing $[\mathrm{R}]_{0}$. If $\mathrm{k}_{\mathrm{a}}$ is smaller than $\mathrm{k}_{\mathrm{d}^{\prime}}$, the observed $\mathrm{EC}_{50}$ is lower than the ideal $\mathrm{EC}_{50}$ and this difference also increases upon increasing $[R]^{\text {. }}$

Figure 5 depicts the deviation of the observed $\mathrm{EC}_{50}$ from the ideal $\mathrm{EC}_{50}$. This figure again shows that the deviation depends heavily on $[\mathrm{R}]_{0}$. The higher the reciprocal of the observed $\mathrm{EC}_{50}$ (1/observed $\left.E C_{50}\right)$ is above one, the higher this deviation is. Additionally, the more $k_{a}$ deviates from $k_{d^{\prime}}$ the more the observed $\mathrm{EC}_{50}$ deviates from the ideal $\mathrm{EC}_{50}$. Figure 5 also shows that the deviation is more pronounced if $\mathrm{k}_{\mathrm{a}}$ is higher than $\mathrm{k}_{\mathrm{d}}$ (1/observed $\mathrm{EC}_{50}$ is above one), compared to the situation in which $\mathrm{k}_{\mathrm{a}}$ is lower than the $\mathrm{k}_{\mathrm{d}}\left(1 /\right.$ observed $\mathrm{EC}_{50}$ is below one).

In the simulation of a competition assay as described above, a limited number of reactions have been incorporated. For example, one molecule of A consumes maximally a single molecule of $\mathrm{R}$. Under the actual experimental conditions of the competition assay, additional reactions cannot be excluded. One of the reactions that has to be considered is the reaction of the product formed out of the antioxidant (PA) with $\mathrm{R}$. Therefore, the simulation was extended, incorporating also the reaction of the product PA with $R$ (equation 8). In the extended simulation, one molecule of A can ultimately consume two molecules of $R$ (the first molecule of $R$ reacts with $A$ and the second molecule of $R$ reacts with $\mathrm{PA}$ ). The total number of molecules of $R$ that finally can be consumed by one molecule of $A$ is described by the total scavenging capacity (CAP). The CAP of the antioxidant is one in the simulation in which one molecule of A consumes only one molecule of $\mathrm{R}$ (the non-extended simulation) and two in the extended simulation.

The influence of $[\mathrm{R}]_{0}$ on the $\mathrm{EC}_{50}$ is depicted in figure 6 for antioxidants with a capacity of two. The $E C_{50}$ crosses the asymptote $\left(E C_{50}=0.5 \cdot[R]_{0}\right)$ that was found in figure 4 , which gave the minimal $E C_{50}$ of an antioxidant with a capacity of one. If $k_{a}$ (reaction rate constant of $A$ with $R$, equation 2 ) and $\mathrm{k}_{\mathrm{a} 2}$ (reaction rate constant of PA with $\mathrm{R}$, equation 8) are $5 \mathrm{mM}^{-1} \mathrm{~s}^{-1}$, the $\mathrm{EC}_{50}$ is almost independent of $[R]_{0}$. However, at higher $k_{a 2}$ an initially decrease in $E C_{50}$ (if $k_{a 2}>k_{a}$ ) is observed at increasing $[R]_{0}$. After reaching a minimum, the $\mathrm{EC}_{50}$ increases slowly by increasing $[\mathrm{R}]_{0}$ toward the asymptote $\mathrm{EC}_{50}=$ $0.25 \cdot[R]_{0}$. The equation of the asymptote can be linked to the total scavenging capacity of the antioxidant (CAP). Equation 13 gives the minimal $\mathrm{EC}_{50}$ of an antioxidant with a particular CAP.

$E C_{50}=\frac{0.5}{C A P} \cdot[R]_{0}$

Equation 13

\section{Discussion}

Competition assays are performed to describe the antioxidant activity and to rank the antioxidants. The $k_{a}$ is the ultimate denominator of the efficacy of scavenging antioxidants. It represents the reaction rate constant of the reaction of the antioxidant $(A)$ with the reactive species $(R)$ it has to scavenge (equation 2). It is generally assumed that the $\mathrm{EC}_{50}$ found in competition assays is linearly proportional to $\mathrm{k}_{\mathrm{a}}$. The $\mathrm{EC}_{50}$ is the primary outcome of most competition experiments for assessing antioxidant activity. Therefore, the focus of this study is on the $\mathrm{EC}_{50}$ rather than on the $\mathrm{k}_{\mathrm{a}}$. 


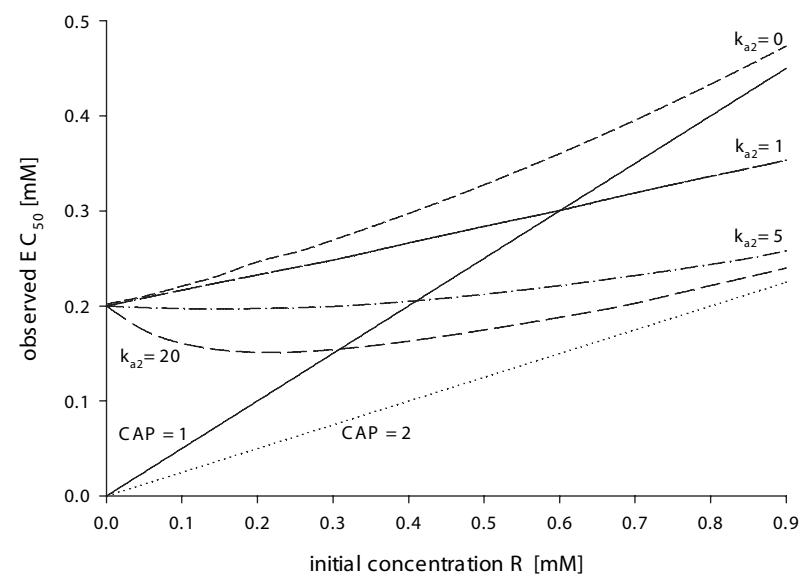

Figure 6. The effect of the oxidation product PA on the observed antioxidant activity. The antioxidant activity is depicted as the observed $E C_{50}$. The oxidation product (PA) formed reacts also with $R$ (second order reaction rate constant $k_{a 2}$ ) and PPA is formed. PA, $A$ and $D$ will compete for $R$. The reaction of PA with $R$ has a stoichiometry of one. The reaction rate constants used in the simulation are $k_{a}=5 \mathrm{mM}^{-1} \mathrm{~s}^{-1}$ and the $k_{d}=1 \mathrm{mM}^{-1} \mathrm{~s}^{-1}$ and $[D]_{0}=1 \mathrm{mM}$. The asymptotes denoted by ' $C A P=l^{\prime}$ and ' $C A P=2^{\prime}$ are depicted as dashed lines. If PA does not react with $R\left(k_{a 2}=0\right)$, the activity of the antioxidant reaches the asymptote ' $C A P=1$ '. If $P A$ does react with $R$, the $E C_{50}$ crosses the asymptote ' $C A P=1$ ' at increasing $[R]$. The higher the reaction rate of $P A$ with $R$, the closer the observed $E C_{50}$ approaches the second asymptote ' $C A P=2$ '. At a high $k_{a z}$ the observed $E C_{50}$ first decreases with increasing $[R]_{0}$ and then increases.

The simulation of a competition assay with known $k_{a^{\prime}} k_{d}$ and $[D]_{0}$ shows that changing $k_{a}$ does not result in a proportional change in observed $\mathrm{EC}_{50^{\prime}}$ as described in the results section. The observed $\mathrm{EC}_{50}$ depends heavily on the experimental conditions, particularly on the initial concentration of the reactive species (figure 3). In general, the observed $\mathrm{EC}_{50}$ of all antioxidants shifts at increasing $[\mathrm{R}]_{0}$ toward the same value, the concentration of $D$ (figure 4). In practice, the efficacy of an antioxidant is not accurately determined and differences between antioxidants are underestimated.

The observed $\mathrm{EC}_{50^{\prime}}$ obtained as is shown in figure 3, was compared to the ideal $\mathrm{EC}_{50^{\prime}}$ obtained via equation 12. The deviation, shown in figure 5 , of the observed $\mathrm{EC}_{50}$ from the ideal $\mathrm{EC}_{50}$ is caused by the consumption of the antioxidant and the detector during the experiment. This is visualized in figure 7. In every experiment, the concentrations of $A$ and $D$ decrease owing to their reaction with R. If $k_{a}$ is larger than $k_{d^{\prime}}$ the relative decrease in [A] is greater than the relative decrease in [D] (see figure 2). This difference in consumption of $D$ and $A$ means that the reaction rate of $D$ with $R\left(v_{d}\right)$ is less reduced than the reaction rate of $A$ with $R\left(v_{a}\right)$ (equations 4 and 5 ). These different reaction rates $\left(v_{d}\right.$ lower than $\left.v_{a}\right)$ lead to an observed $\mathrm{EC}_{50}$ that is higher than the ideal $\mathrm{EC}_{50}$ calculated from the ratio of $k_{a}$ and $k_{d}$. This explains the difference between the observed $E C_{50}$ and the ideal $E C_{50}$ (figure 5). 

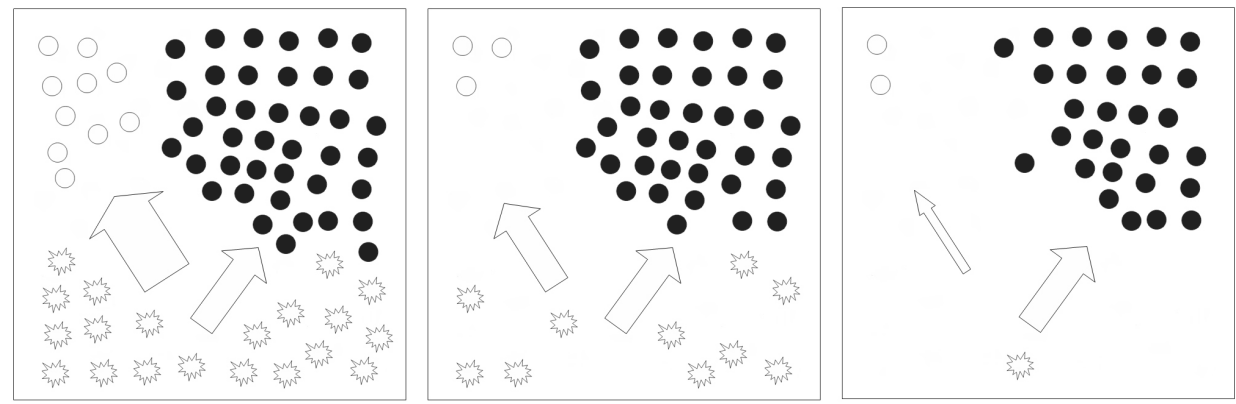

Figure 7. Schematic representation of the conditions at the start, approximately halfway and at the end of a competition assay. In this representation, the antioxidant (depicted as open circles, initial concentration of $0.25 \mathrm{mM}$ ) reacts faster with the reactive species (depicted as stars, initial concentration $0.5 \mathrm{mM}$ ) than the detector (depicted as closed circles, initial concentration $1 \mathrm{mM})\left(k_{a}=20 \mathrm{mM}^{-1} \mathrm{~s}^{-1}\right.$ and $\left.k_{d}=1 \mathrm{mM}^{-1} \mathrm{~s}^{-1}\right)$. This is the $E C_{50}$ condition (half of the reactive species reacts with the antioxidant and the other half reacts with $D)$. At the start of the experiment, the reaction rate of the antioxidant $\left(v_{d}\right)$ is higher than the reaction rate of the detector $\left(v_{d}\right)$. This is indicated by a wider arrow, pointing toward the antioxidants. As a result of the high reaction rate of the antioxidant, the antioxidant is consumed to a larger extent than the detector. Consequently, $v_{a}$ approaches $v_{d}$ halfway through the reaction. At the end of the reaction, the concentration of antioxidant is so low that $v_{d}$ is much higher than $v_{a}$. This shows that $v_{a}$ and $v_{d}$ are not equally reduced during the competition assay. The fundamental problem is that during a competition assay the concentration of the antioxidant and the concentration of the detector are not reduced to the same extent. This has to be taken into account when antioxidant activity is assessed.

The extent of the deviation depends on the concentration of $[R]_{0}$ relative to $[A]_{0}$ and $[D]_{0}$. Very low $[R]_{0}$ will not change the ratio of $[A]$ and $[D]$ significantly. With low $[R]_{0^{\prime}}$ the obtained observed $E C_{50}$ will be close to the ideal $E C_{50}$. High $[R]_{0}$ might change the ratio of $[A]$ and $[D]$ significantly during the competition experiment, which leads to significantly higher (if $k_{a}>k_{d}$ ) or lower (if $k_{a}<k_{d}$ ) observed $\mathrm{EC}_{50}$ values, relative to the ideal $\mathrm{EC}_{50}$.

In addition to $[R]_{0^{\prime}}$ also the ratio of $k_{a}$ and $k_{d}$ influences the extent of the deviation of the observed $\mathrm{EC}_{50}$ from the ideal $\mathrm{EC}_{50}$. If $\mathrm{k}_{\mathrm{a}}$ is much higher than $\mathrm{k}_{\mathrm{d}^{\prime}}$ the observed $\mathrm{EC}_{50}$ levels off and the observed $\mathrm{EC}_{50}$ becomes dependent only on $[\mathrm{R}]_{0}$. Under this condition, $\mathrm{R}$ will react only with $\mathrm{D}$, after $\mathrm{A}$ has completely been consumed. By definition, at the observed $\mathrm{EC}_{50}$ half of the amount PD is formed compared to the situation in which no $A$ is present. This indicates that (if $k_{a}>>k_{d}$ ) at the observed $\mathrm{EC}_{50}$ half of $[\mathrm{R}]_{0}$ will react with $\mathrm{D}$. The other half of $[\mathrm{R}]_{0}$ has reacted with $\mathrm{A}$. If $\mathrm{k}_{\mathrm{a}}$ is much higher than $\mathrm{k}_{\mathrm{d}^{\prime}}$ the observed $\mathrm{EC}_{50}$ of the antioxidant is equal to half of the concentration of $[\mathrm{R}]_{0}$. Under these conditions, the observed $\mathrm{EC}_{50}$ reflects the capacity rather than the $\mathrm{k}_{\mathrm{a}}$ of the antioxidant.

The product formed out of the antioxidant (PA) is often not inert and may also react with the reactive species. For chrysin for example, it has been reported that the product has an antioxidant effect that even exceeds that of chrysin itself [6]. Therefore, the competition assay was extended by changing the capacity of the antioxidant from one to two. The product formed from the antioxidant (PA) also reacts with $\mathrm{R}$. The observed $\mathrm{EC}_{50}$ is then also determined by the antioxidant activity of the product of the antioxidant (figure 6). 
How to determine the accuracy of a given $E C_{50}$

The accuracy of a certain antioxidant activity, i.e. the deviation in observed $\mathrm{EC}_{50^{\prime}}$ can be predicted if $[R]_{0^{\prime}}[D]_{0}$ and the observed $E C_{50}$ are known and a CAP of one is used (figure 8). An observed $E C_{50}$ below half of $[\mathrm{R}]_{0}$ (observed $\mathrm{EC}_{50} /[\mathrm{R}]_{0}$ smaller than 0.5 ) indicates that the product of the antioxidant is surely involved in the assay. The reported activity is not due solely to the antioxidant itself, but also to the product formed from the antioxidant. If $[R]_{0}$ is larger than $[D]_{0}\left([D]_{0} /[R]_{0}\right.$ smaller than one), the assay is not a properly designed competition assay, because there is a relative excess of reactive species. If no antioxidant is added to the assay, D will be completely consumed, whereas some $\mathrm{R}$ still remains.

Between these boundaries (observed $\mathrm{EC}_{50} /[\mathrm{R}]_{0}<0.5$ and $[\mathrm{D}]_{0} /[\mathrm{R}]_{0}<1$ ), drawn in figure 8 , competition of $A$ and $D$ for $R$ is possible. In the extraordinary case that $k_{d}$ equals to $k_{a^{\prime}}$ ideal competition will take place; the observed $\mathrm{EC}_{50}$ equals the ideal $\mathrm{EC}_{50}$. If the observed $\mathrm{EC}_{50}$ is not located on this $0 \%$ line, the observed $\mathrm{EC}_{50}$ deviates from the ideal $\mathrm{EC}_{50}$. The extent of the deviation increases with decreasing ratio $[D]_{0} /[R]_{0}$. Therefore, $[R]_{0}$ should be as low as possible to minimize this deviation. However, if $[R]_{0}$ is reduced, the product (PD) formed will decrease and this negatively affects the precision of the experiment. This product PD is mostly used to monitor a competition assay. These opposite effects of $[R]_{0}$ (i.e. low $[R]_{0}$ for low deviation, high $[R]_{0}$ for high precision) indicate that an optimum has to be found.

In the next paragraphs, several frequently used antioxidant assays are reviewed with respect to the issues addressed in the present paper. When for an antioxidant a high deviation of the observed $\mathrm{EC}_{50}$ is found, a strategy to improve the competition assay is given. Several examples are presented in table 1 and figure 8.

Figure 8. A graphical tool to estimate the accuracy of the antioxidant efficacy obtained in a competition experiment. The ratio of the observed $E C_{50}$ and the initial concentration of radical, $[R]_{0}$ (both in molar), is plotted against the ratio of the initial concentration of detector ( $\left.[D]_{0}\right)$ and the initial amount of $[R]_{0}$ (both also in molar). The dotted lines give the actual deviation of the observed $E C_{50}$ versus the ideal $E C_{50}$. It is assumed that competition takes place. If the point obtained is located in the gray area on the left, this indicates that the experiment is ill designed; the initial concentration of the radical exceeds that of the detector. The gray area in the bottom indicates conditions under which oxidation products of the antioxidant have to contribute to the assay. The numbers refer to the antioxidants and assays described in table 1. 


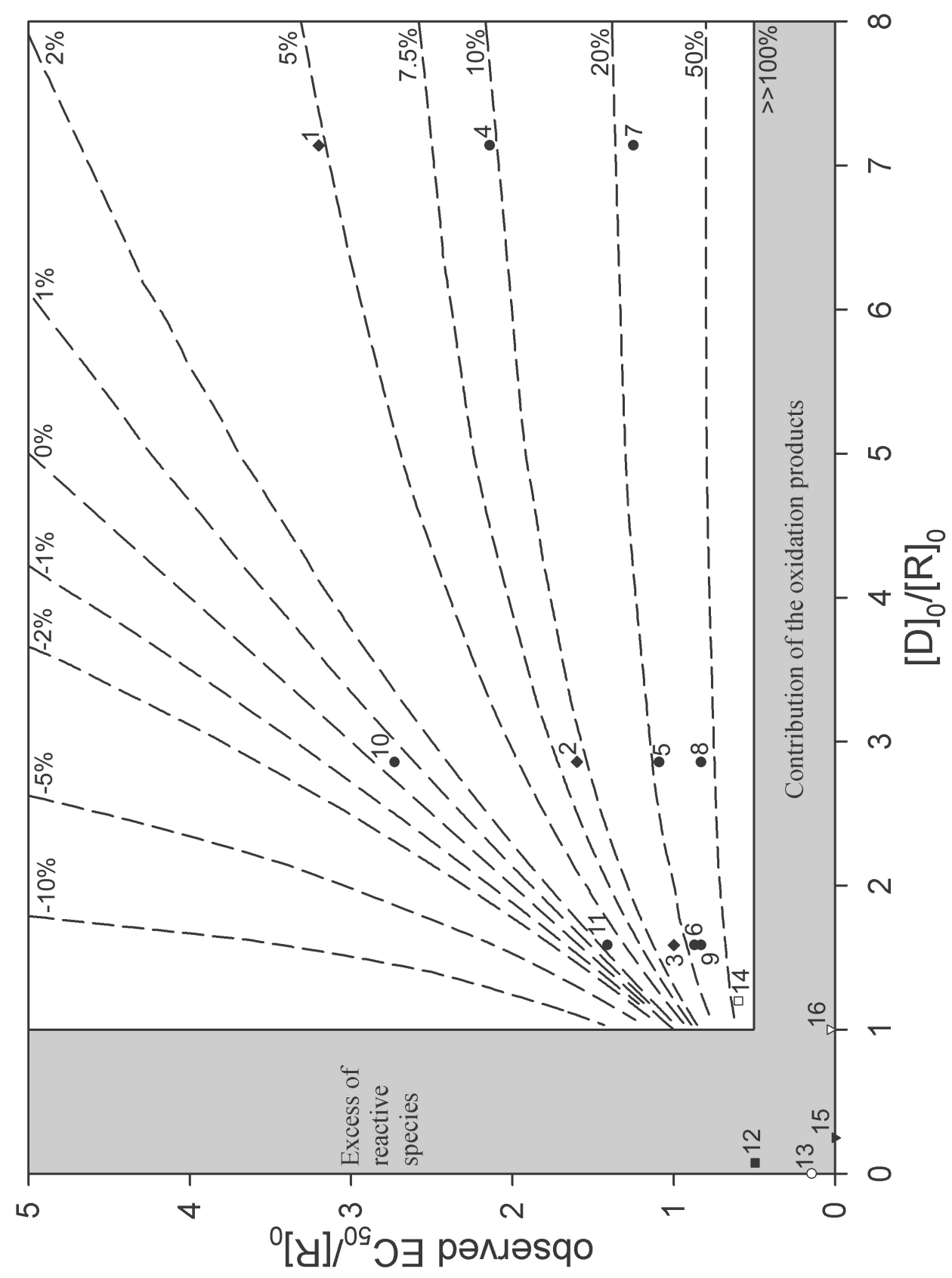


Table 1: Review of the activity of some antioxidants according to the tool given in figure 8.

\begin{tabular}{|c|c|c|c|c|c|c|c|c|}
\hline Antioxidant & Detector & $\begin{array}{l}\text { Reactive } \\
\text { species }\end{array}$ & $\begin{array}{l}{[\mathrm{D}]_{0} \text { in }} \\
\mathrm{mM}\end{array}$ & $\begin{array}{l}{[\mathrm{R}]_{0} \text { in }} \\
\mathrm{mM}\end{array}$ & $\begin{array}{l}\mathrm{EC}_{50} \text { in } \\
\mathrm{mM}\end{array}$ & $\begin{array}{l}\text { Deviation } \\
\text { (\%) }\end{array}$ & $\begin{array}{l}\text { Point } \\
\text { in } \\
\text { fig. } 8\end{array}$ & \\
\hline GSH & LA & $\mathrm{HOCl}$ & 1 & 0.14 & 0.45 & $2-5$ & 1 & \\
\hline GSH & LA & $\mathrm{HOCl}$ & 1 & 0.35 & 0.56 & $7.5-10$ & 2 & \\
\hline GSH & LA & $\mathrm{HOCl}$ & 1 & 0.63 & 0.63 & $10-20$ & 3 & \\
\hline GSH & MET & $\mathrm{HOCl}$ & 1 & 0.14 & 0.30 & $7.5-10$ & 4 & \\
\hline GSH & MET & $\mathrm{HOCl}$ & 1 & 0.35 & 0.38 & $20-50$ & 5 & \\
\hline GSH & MET & $\mathrm{HOCl}$ & 1 & 0.63 & 0.52 & $20-50$ & 6 & \\
\hline TNB & MET & $\mathrm{HOCl}$ & 1 & 0.14 & 0.18 & $20-50$ & 7 & \\
\hline TNB & MET & $\mathrm{HOCl}$ & 1 & 0.35 & 0.29 & $20-50$ & 8 & \\
\hline TNB & MET & $\mathrm{HOCl}$ & 1 & 0.63 & 0.55 & $20-50$ & 9 & \\
\hline LA & MET & $\mathrm{HOCl}$ & 1 & 0.35 & 0.96 & $0-1$ & 10 & \\
\hline LA & MET & $\mathrm{HOCl}$ & 1 & 0.63 & 0.89 & $2-5$ & 11 & \\
\hline LA & BSA & $\mathrm{HOCl}$ & $0.015^{\mathrm{a}}$ & 0.2 & 0.1 & $>>100$ & 12 & [29] \\
\hline LA & Catalase & $\mathrm{HOCl}$ & 0.0166 & 6 & 0.86 & $>>100$ & 13 & [30] \\
\hline LA & $a_{1}-A P$ & $\mathrm{HOCl}$ & $0.06^{b}$ & 0.05 & 0.03 & $>50$ & 14 & [31] \\
\hline Quercetin & Taurine & $\mathrm{HOCl}$ & 150 & 600 & 0.086 & $>>100$ & 15 & [32] \\
\hline Trolox & Taurine & $\mathrm{HOCl}$ & 150 & 600 & 0.56 & $>>100$ & & [32] \\
\hline GSH & Taurine & $\mathrm{HOCl}$ & 150 & 600 & 0.67 & $>>100$ & & [32] \\
\hline BHT & Taurine & $\mathrm{HOCl}$ & 150 & 600 & 1.71 & $>>100$ & & [32] \\
\hline Gallic acid & Taurine & $\mathrm{HOCl}$ & 150 & 600 & 0.28 & $>>100$ & & [32] \\
\hline LA & Luminol & $\mathrm{HOCl}$ & 0.25 & 0.025 & 0.006 & $>>100$ & & [33] \\
\hline Quercetin & DHR & $\mathrm{HOCl}$ & 0.005 & 0.005 & 0.0011 & $>>100$ & & [34] \\
\hline Chrysin & DHR & $\mathrm{HOCl}$ & 0.005 & 0.005 & 0.0039 & $20-50$ & & [34] \\
\hline LA & DHR & $\mathrm{HOCl}$ & 0.005 & 0.005 & 0.0024 & $>>100$ & & [34] \\
\hline Quercetin & Tyrosine & PON & 1 & 1 & 0.03 & $\gg>100$ & 16 & [35] \\
\hline Trolox & Tyrosine & PON & 0.1 & 0.5 & 0.05 & $>>100$ & & [36] \\
\hline Trolox & Tyrosine & PON & 1 & 1 & 0.098 & $>>100$ & & [37] \\
\hline GSH & Tyrosine & PON & 1 & 1 & 0.194 & $>>100$ & & [37] \\
\hline Uric acid & Tyrosine & PON & 1 & 1 & 0.064 & $>>100$ & & [37] \\
\hline 2-coumaric acid & Tyrosine & PON & 0.1 & 0.5 & 0.06 & $>>100$ & & [36] \\
\hline 4-coumaric acid & Tyrosine & PON & 0.1 & 0.5 & 0.055 & $>>100$ & & [36] \\
\hline Ferulic acid & Tyrosine & PON & 0.1 & 0.5 & 0.046 & $>>100$ & & [36] \\
\hline Ascorbate & Tyrosine & PON & 1 & 1 & 0.212 & $>>100$ & & [37] \\
\hline Quercetin & DHR & PON & 0.005 & 0.0006 & 0.00053 & $>50$ & & [38] \\
\hline Uric acid & DHR & PON & 0.05 & 0.0006 & 0.0025 & $>>100$ & & [7] \\
\hline 4-coumaric acid & DHR & PON & 0.005 & 0.0006 & 0.00083 & $10-20$ & & [38] \\
\hline Kaempferol & DHR & PON & 0.005 & 0.0006 & 0.00035 & $>50$ & & [38] \\
\hline Hydroxytyrosol & DHR & PON & 0.005 & 0.0006 & 0.0033 & $1-2$ & & {$[16]$} \\
\hline Oleuropein & DHR & PON & 0.005 & 0.0006 & 0.0021 & $2-5$ & & [16] \\
\hline Catechol & DHR & PON & 0.005 & 0.0006 & 0.0027 & $2-5$ & & [16] \\
\hline Epicatechin & DHR & PON & 0.005 & 0.0003 & 0.00062 & $10-20$ & & [39] \\
\hline Epigallocatechin & DHR & PON & 0.005 & 0.0003 & 0.00074 & $7.5-10$ & & [39] \\
\hline Epicatechin gallate & DHR & PON & 0.005 & 0.0003 & 0.00039 & $10-20$ & & [39] \\
\hline
\end{tabular}




\begin{tabular}{|c|c|c|c|c|c|c|c|}
\hline $\begin{array}{l}\text { Epigallocatechin } \\
\text { gallate }\end{array}$ & DHR & PON & 0.005 & 0.0003 & 0.00033 & $20-50$ & [39] \\
\hline Phloridzin & DHR & PON & 0.005 & 0.0006 & 0.055 & $0-1$ & [40] \\
\hline Phloretin & DHR & PON & 0.005 & 0.0006 & 0.0031 & $2-5$ & [40] \\
\hline $\begin{array}{l}\text { 2,4,6-trihydroxy- } \\
\text { acetophenone }\end{array}$ & DHR & PON & 0.005 & 0.0006 & 0.0055 & $0-1$ & [40] \\
\hline $\begin{array}{l}\text { 2,6-dihydroxy- } \\
\text { acetophenone }\end{array}$ & DHR & PON & 0.005 & 0.0006 & 0.0078 & $0-1$ & [40] \\
\hline $\begin{array}{l}\text { 5-methyl-tetrahy- } \\
\text { drofolic acid }\end{array}$ & DHR & PON & 0.005 & 0.0006 & 0.0009 & $10-20$ & [41] \\
\hline Tetrahydrofolic acid & DHR & PON & 0.005 & 0.0006 & 0.0015 & $7.5-10$ & {$[41]$} \\
\hline $\begin{array}{l}\text { 7,8-Dihydrofolic } \\
\text { acid }\end{array}$ & DHR & PON & 0.005 & 0.0006 & 0.0024 & $2-5$ & [41] \\
\hline Rutin & DHR & PON & 0.005 & 0.0003 & 0.00047 & $10-20$ & [42] \\
\hline Mono-HER & DHR & PON & 0.005 & 0.0003 & 0.00029 & $>>100$ & [42] \\
\hline Di-HER & DHR & PON & 0.005 & 0.0003 & 0.0025 & $2-5$ & [42] \\
\hline Tri-HER & DHR & PON & 0.005 & 0.0003 & 0.162 & $0-1$ & [42] \\
\hline Kaempferol & Luminol & $\mathrm{O}_{2}^{-}$ & 8 & $0.001^{c}$ & 0.005 & $2-5$ & [43] \\
\hline Quercetin & Luminol & $\mathrm{O}_{2}^{-}$ & 8 & $0.001^{c}$ & 0.0075 & $2-5$ & [43] \\
\hline Cyanidin & Luminol & $\mathrm{O}_{2}^{-}$ & 0.4 & $0.25^{c}$ & 1.5 & $>10$ & [44] \\
\hline Lutein & Lucigenin & $\mathrm{O}_{2}^{-}$ & 0.15 & $0.9^{c}$ & 0.1 & $>>100$ & {$[45]$} \\
\hline Tempo & Lucigenin & $\mathrm{O}_{2}^{-}$ & 0.15 & $0.9^{c}$ & 0.017 & $>>100$ & {$[45]$} \\
\hline BHT & Cyt C & $\mathrm{O}_{2}^{-}$ & 0.01 & $7.5^{*} 10^{-8 c}$ & 0.026 & $0-1$ & [11] \\
\hline Ferulic acid & Cyt C & $\mathrm{O}_{2}^{-}$ & 0.01 & $7.5^{*} 10^{-8 c}$ & 0.058 & $0-1$ & [11] \\
\hline BHT & NBT & $\mathrm{O}_{2}^{-}$ & 0.017 & $0.026^{d}$ & 0.14 & $>>100$ & [9] \\
\hline $\mathrm{BHA}$ & NBT & $\mathrm{O}_{2}^{-}$ & 0.017 & $0.026^{d}$ & 0.2 & $>>100$ & [9] \\
\hline a-tocopherol & NBT & $\mathrm{O}_{2}^{-}$ & 0.017 & $0.026^{d}$ & 0.077 & $>>100$ & [9] \\
\hline Ascorbic acid & NBT & $\mathrm{O}_{2}^{-}$ & 0.05 & $0.48^{d}$ & 0.26 & $>>100$ & {$[8]$} \\
\hline Cyanidin & NBT & $\mathrm{O}_{2}^{-}$ & 0.05 & $0.48^{\mathrm{d}}$ & 0.024 & $>>100$ & {$[8]$} \\
\hline a-tocopherol & NBT & $\mathrm{O}_{2}^{-}$ & 0.05 & $0.026^{d}$ & 2.3 & $5-10$ & {$[10]$} \\
\hline BHT & NBT & $\mathrm{O}_{2}^{-}$ & 0.05 & $0.026^{d}$ & 6.8 & $>10$ & [10] \\
\hline Gallic acid & Nitrite & $\mathrm{O}_{2}^{-}$ & 1 & $1.2^{c}$ & 0.0021 & $>>100$ & {$[46]$} \\
\hline Trolox & DOR & $\mathrm{OH}$ & 2.8 & $0.01^{e}$ & 0.75 & $0-1$ & [47] \\
\hline Mannitol & DOR & $\mathrm{OH}$ & 2.8 & $0.01^{e}$ & 3.272 & $0-1$ & [48] \\
\hline Quercetin & DOR & $\cdot \mathrm{OH}$ & 2.8 & $0.01^{e}$ & 1.34 & $0-1$ & [49] \\
\hline Trolox & Luminol & $\cdot \mathrm{OH}$ & 0.02 & n.a. & 0.0007 & $>>100$ & [33] \\
\hline Quercetin & DMSO & $\mathrm{OH}$ & 0.2 & $0.1^{\dagger}$ & 0.074 & $20-50$ & [50] \\
\hline Dithiothreitol & Coumarin & $\mathrm{OH}$ & 0.04 & 0.004 & 0.016 & $2-5$ & [51] \\
\hline
\end{tabular}

$\mathrm{HOCl}=$ hypochlorous acid, $\mathrm{O}_{2}^{-=}=$superoxide, $\mathrm{OH}=$ hydroxyl radical, $\mathrm{PON}=$ peroxynitrite, $\mathrm{GSH}=$ reduced glutathione,

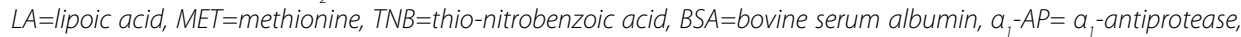
$B H T=$ butylated hydroxytoluene, $B H A=$ butylated hydroxyanisole, DHR=dihydrorhodamine, HER=hydroxyethyl rutoside, $N B T=$ nitroblue tetrazolium, $D O R=$ deoxyribose, n.a.=not available

a $[B S A]=1 \mathrm{mg} / \mathrm{ml}$, molecular weight is $66 \mathrm{kDa}$.

${ }^{b}$ Based on $[R]_{0}$ needed to completely inhibit a, -AP activity.

${ }^{c}$ Assuming that $1 \mathrm{U} / \mathrm{m} /$ xantine oxidase produces $1 \mu \mathrm{mol}$ superoxide per minute.

${ }^{d}$ Assuming that $1.6 \mathrm{nmol}$ superoxide is produced per minute per $\mu \mathrm{mol}$ phenazine methosulphate [52].

e Based on the production of thiobarbituric acid in the absence of antioxidant from the original publication of Halliwell [13]. ${ }^{f}$ Estimated from the maximum fluorescence signal. 


\section{Hypochlorous acid scavenging}

The activity of antioxidants to scavenge hypochlorous acid ( $\mathrm{HOCl}$ ) can be determined in several assays, which will be named after the detector in the assay.

The $\mathrm{HOCl}$ scavenging activity of reduced glutathione (GSH) was evaluated in a competition with lipoic acid $\left(L A,[D]_{0}=1 \mathrm{mM}\right)$ as detector. An initial concentration of $0.63 \mathrm{mM} \mathrm{HOCl}\left([\mathrm{R}]_{0}=0.63 \mathrm{mM}\right)$ resulted in an obtained $\mathrm{EC}_{50}$ of $0.63 \mathrm{mM}$. Figure 8 indicates that with these experimental conditions $\left([\mathrm{D}]_{0} /[\mathrm{R}]_{0}=1.6\right.$ and observed $\left.\mathrm{EC}_{50} /[\mathrm{R}]_{0}=1\right)$ the observed $\mathrm{EC}_{50}$ deviates $10-20 \%$ from the ideal $\mathrm{EC}_{50}$ (point 3 in figure 8). This high positive deviation can have two possible causes: either [R] (which is the initial concentration of $\mathrm{HOCl}$ ) is too high, or the detector ( $\mathrm{LA}$ ) reacts too slowly with the reactive species (table 2). If [R] is decreased to $0.35 \mathrm{mM}$ or $0.14 \mathrm{mM}$, the deviation decreases respectively to 7.5-10\% (point 2 in figure 8 ) or 2-5\% (point 1 in figure 8 ). This shows that in this case reduction of [R] (the initial concentration of $\mathrm{HOCl}$ ) solves the problem (figure 9).

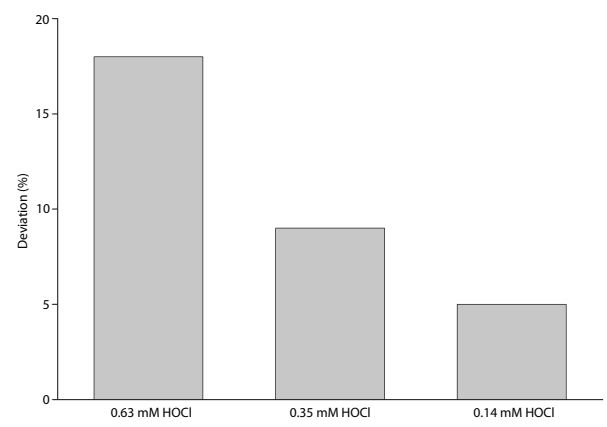

Figure 9. Example of improving a competition assay by reducing the initial concentration of the reactive species. The deviations are estimated from figure 8. The deviation of the observed $E C_{50}$ from the ideal $E C_{50}$ of GSH in the methionine assay at a concentration $0.63 \mathrm{mM} \mathrm{HOCl}$ is too high (point 3 in figure 8). The possible cause of the high positive deviation is, according to table 2, a concentration of $[R]_{0}$ that is too high. The solution is to decrease $[R]_{0}$ (table 2). By reducing the concentration of $\mathrm{HOCl}$ to $0.35 \mathrm{mM}$ or $0.14 \mathrm{mM}$, the deviation drastically decreases (points 2 and 1 in figure 8). This example shows that the accuracy is indeed improved by reducing the initial concentration of the reactive species.

There are two fundamental problems in the $\mathrm{HOCl}$ scavenging assay of the antioxidant lipoic acid and the detectors catalase (point 13, fig. 8) or albumin (point 12, fig 8). There is an excess of reactive species and oxidation products contribute to the obtained $\mathrm{EC}_{50}$. If a different detector is used ( $\mathrm{a}_{1}-\mathrm{AP}$, point 14 , fig. 8), both problems are solved. The deviation of the observed $\mathrm{EC}_{50}$ is reduced, although it is still above 50\% (figure 10). If methionine is used as detector, the deviation decreases to 2-5\% (point 11 , fig 8). There is still a positive deviation, which could be reduced by decreasing $[\mathrm{R}]_{0}$ (see table 2 ). Further decreasing $[\mathrm{R}]_{0}$ (lowering the initial concentration of $\mathrm{HOCl}$ from $0.63 \mathrm{mM}$ to $0.35 \mathrm{mM}$ ) results in an observed $\mathrm{EC}_{50}$ which deviates only 0-1\% from the ideal $\mathrm{EC}_{50}$ (point 10, fig 8). 


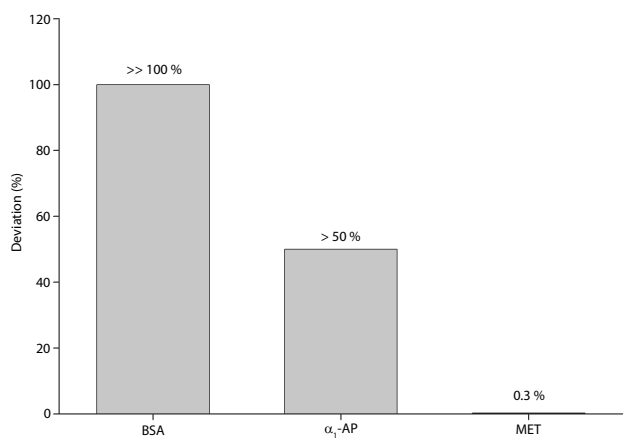

Figure 10. Example of improving a competition assay by choosing a detector that has a reaction rate similar to that of the antioxidant for the reactive species. The deviations are estimated from figure 8 . The high deviation of the observed $E C_{50}$ from the ideal $E C_{50}$ of $L A$ in the BSA assay is due to the contribution of oxidation products (point 12 in figure 8). The possible cause according to table 2 is a detector that reacts too slowly. The solution is to choose a different detector. With another detector ( $a_{1}$-AP, point 14 in figure 8), the problem shifts to a high positive deviation. The possible cause according to table 2 is that $A$ reacts faster with $R$ than $D$ reacts with $R$. The solution is to choose another detector. By choosing the right detector the deviation can be drastically decreased (MET as detector, point 10 in figure 8). This example shows that the accuracy can be improved by choosing the right detector.

Table 2: Problem-solving strategy for competition assays.

\begin{tabular}{|c|c|c|}
\hline Problem according to figure $8:$ & Possible cause: & Solution: \\
\hline \multirow[t]{2}{*}{ Excess of reactive species } & - $[D]_{0}$ is too low & - Increase $[D]_{0}$ \\
\hline & $-[\mathrm{R}]_{0}$ is too high & - Decrease $[\mathrm{R}]_{0}$ \\
\hline $\begin{array}{l}\text { Contribution of oxidation products } \\
(>>100 \%)\end{array}$ & - D reacts too slowly with $R$ & - Use a detector that reacts faster with $R$ \\
\hline \multirow[t]{2}{*}{ High negative deviation } & - $[\mathrm{R}]_{0}$ is too high & - Decrease $[R]_{0}$ \\
\hline & $\begin{array}{l}\text { - A reacts more slowly with } R \text { than } \\
\text { does } D\end{array}$ & $\begin{array}{l}\text { - Use a detector that reacts more slowly } \\
\text { with } \mathrm{R}\end{array}$ \\
\hline \multirow[t]{2}{*}{ High positive deviation } & $-[\mathrm{R}]_{0}$ is too high & - Decrease $[\mathrm{R}]_{0}$ \\
\hline & - A reacts faster with $R$ than does $D$ & - Use a detector that reacts faster with $R$ \\
\hline
\end{tabular}




\section{Peroxynitrite scavenging assay}

A frequently used assay to measure scavenging of peroxynitrite (PON) derived radicals is the dihydrorhodamine assay (DHR) developed by Kooy et al. [7]. In this assay, the detector molecule dihydrorhodamine $\left([D]_{0}=50 \mu \mathrm{M}\right)$ reacts with PON derived radicals $\left([R]_{0}=0.6 \mu \mathrm{M}\right)$ and the fluorescent product rhodamine (which is $\mathrm{PD}$ ) is formed. The obtained $\mathrm{EC}_{50}$ of phenolic compounds is in the order of $1 \mu \mathrm{M}$ to $100 \mu \mathrm{M}$ (table 1). This means that in general, $[\mathrm{R}]_{0}$ is substantially lower than $[\mathrm{D}]_{0}$ and $[\mathrm{A}]_{0}$. Therefore, the obtained $\mathrm{EC}_{50}$ has a reasonable accuracy, although for some individual compounds a high deviation is found (table 1).

Another assay to determine the antioxidant activity for PON is the tyrosine assay. The deviation obtained in figure 8 for all compounds is much higher than 100\%. The most striking observation is that for all compounds the observed $\mathrm{EC}_{50}$ was much lower than the initial concentration of PON $\left([R]_{0}\right)$. This indicates that oxidation products (PA) contribute to the observed antioxidant activity. It should be realized that in the tyrosine assay other reactions probably also play a role e.g. scavenging of tyrosine radical. This questions the validity of the assay to determine PON scavenging.

\section{Superoxide scavenging assay}

Superoxide radical scavenging is usually determined in an assay in which the radical is formed continuously by xantine and xantine oxidase or by phenazine methosulphate. The antioxidant and detector (e.g. nitroblue tetrazolium [8-10] or cytochrome C $[11,12])$ compete for the formed superoxide. The formation of the oxidized detector is monitored in time. Because radicals are continuously formed in time, $[\mathrm{R}]_{0}$ is not defined. Based on the activity of xantine oxidase (from 5 $\mathrm{nU} / \mathrm{ml}$ to $0.9 \mathrm{U} / \mathrm{ml}$ ) and the time of the assay requires (from one to thirty minutes), the amount of superoxide formed was calculated. Based on the amount of superoxide formed $\left([R]_{0}\right)$, the $[D]_{0}$ and the $\mathrm{EC}_{50^{\prime}}$ the deviation has been determined. This revealed that the accuracy of the assay varied widely, from an excess of reactive species to a contribution of oxidation products (table 1).

The possibility in most of the superoxide scavenging assays of monitoring the formation of PD in time offers an additional check on the validity of the antioxidant assay. The formation of PD in time has to be constant. This results in a straight line if the absorbance of the reaction mixture is plotted against time. Deviation from the straight line is indicative for problems such as a substantial consumption of $\mathrm{D}$ or $\mathrm{A}$ resulting in the deviations addressed in the present paper.

In the competition assays reviewed in table 1 , the most critical factor appeared to be $[R]_{0}$. When $[R]_{0}$ is relatively low compared to $[D]_{0^{\prime}}$ a reasonably accurate antioxidant activity was obtained.

\section{Hydroxyl radical scavenging assay}

In the hydroxyl radical scavenging assays, similar to the superoxide scavenging assay, the radical is also continuously formed in time. The combination of ferrous iron and hydrogen peroxide is usually used to generate hydroxyl radicals in the Fenton reaction (equation 14).

$$
\mathrm{H}_{2} \mathrm{O}_{2}+\mathrm{Fe}^{2+} \rightarrow \mathrm{Fe}^{3+}+{ }^{\cdot} \mathrm{OH}+{ }^{-} \mathrm{OH} \quad \text { Equation } 14
$$


The hydroxyl scavenging ability of antioxidants is mostly determined by the method of Halliwell [13] using deoxyribose as detector molecule. In contrast to the superoxide scavenging assay, the reaction of the hydroxyl radical with the detector is not followed in time, but determined after a fixed incubation period. In this assay, the activity of the antioxidant is determined by calculating $k_{a}$. The equation used to calculate $k_{a}$ is based on equations 3 and 4 in the present paper. In calculating $\mathrm{k}_{\mathrm{a}^{\prime}}$ the assumption is made that the concentrations of $\mathrm{A}$ and $\mathrm{D}$ do not change during the incubation period. Thus, although the $\mathrm{EC}_{50}$ is not the outcome measure in this assay, the observed $\mathrm{k}_{\mathrm{a}}$ is as sensitive as the observed $\mathrm{EC}_{50}$ to the problems highlighted in the present paper. After one hour, the concentration of the formed product PD in the assay (around $0.01 \mathrm{mM}$ ) is however far less than the initial concentration deoxyribose $\left([\mathrm{D}]_{0}=2.8 \mathrm{mM}\right)$ and the observed $\mathrm{EC}_{50}$. This indicates that, in this experimental setup, the consumption of $A$ and $D$ is indeed negligible and subsequently the deviation is also negligible.

\section{Hydrogen peroxide scavenging assay}

Hydrogen peroxide is a relatively stable 'reactive' species, which gives the opportunity to monitor the reaction rate $\left(v_{a}\right)$ of hydrogen peroxide with the antioxidant directly. Such a direct kinetic measurement is often superior to indirect determination of $k_{a}$ in a competition assay. From the measured reaction rate $\left(v_{a}\right)$, the $k_{a}$ of the antioxidant can be determined easily.

Thiols react relatively quickly with hydrogen peroxide. Under physiological conditions, the reaction of thiols with hydrogen peroxide is catalyzed by the selenium-dependent GSH-peroxidases. Small organic selenium containing compounds, with ebselen as the prototype, mimic the activity of the peroxidase. The peroxidase activity proceeds by a ter uni ping pong mechanism and the kinetics can be described with the Dalziel equation. In the peroxidase activity of ebselen, there is a substantial difference in the Dalziel constants of thiols [14]. Because the rate constants are determined by directly monitoring hydrogen peroxide, the problems addressed in the present paper do not apply. Occasionally, it is reported that phenolic compounds have the ability to scavenge hydrogen peroxide [15]. Critical examination shows that the reported scavenging of hydrogen peroxide by the compounds is most likely the result of hydrogen peroxide derived radicals, e.g. hydroxyl radicals [16]. Other common assays on hydrogen peroxide scavenging are based on enzyme mediated oxidation of a detector which depends on hydrogen peroxide as cofactor [17]. As mentioned before [18], the presumed hydrogen peroxide scavenging activity is an artifact based on the reaction of the antioxidant with intermediates or on competition between the antioxidant and the detector for the enzyme instead of the reactive species. In general, phenolic compounds do not readily react with hydrogen peroxide, rather they are a potential source for the generation of hydrogen peroxide [19].

\section{Lipid peroxidation}

Lipid peroxidation is a free radical process. It starts with the formation of a lipid radical. This radical reacts quickly with oxygen to form a lipid peroxyl radical. The lipid peroxyl radical (LOO•) abstracts a 
hydrogen atom from a second lipid and a lipid hydroperoxide $(\mathrm{LOOH})$ and a new lipid radical $(\mathrm{L} \cdot)$ are formed. The new lipid radical, in turn, reacts with oxygen and generates another lipid hydroperoxide and a third lipid radical. This propagation or chain reaction can be interrupted by antioxidants. These chain-breaking antioxidants (A) compete with lipid (D) for the lipid peroxyl radical (R). In the lipid peroxidation assays, radicals are often continuously formed in time e.g. via a reducing system together with iron or a thermo labile azo compound.

The most extensively studied process of lipid peroxidation in vitro is low density lipoprotein (LDL) oxidation. The pioneering work of Esterbauer showed that antioxidants induce a lag time in the oxidation of LDL [20]. The length of this lag time is proportional to the concentration of antioxidants. During the lag time, the antioxidant is consumed and upon full consumption of the antioxidant, lipid peroxidation will start. This indicates that no competition between the antioxidants and the radicals takes place, but the capacity of the antioxidant determines the antioxidant activity (see figure 11). This assay can therefore not be used to determine the reaction rate constants of antioxidants, and the tool provided in this study is not suited to evaluate the accuracy of LDL oxidation experiments.
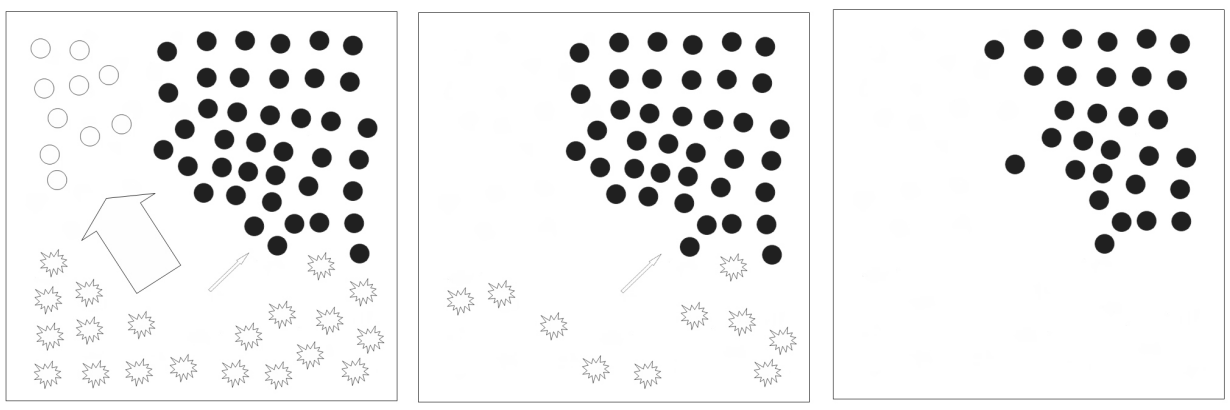

Figure 11. Schematic representation of the conditions at the start, approximately halfway and at the end of a capacity assay. In this representation, the antioxidant (depicted as open circles, initial concentration of $0.25 \mathrm{mM}$ ) reacts very much faster with the reactive species (depicted as stars, initial concentration $0.5 \mathrm{mM}$ ) than the detector (depicted as closed circles, initial concentration $1 \mathrm{mM})\left(k_{a}>>k_{d}\right)$. Halfway the experiment, the antioxidant is completely consumed. The remaining reactive species react with the detector. At the end of the experiment, both the antioxidant and the reactive species are completely consumed. The remaining detector gives the capacity of the antioxidant.

\section{Antioxidant capacity assays}

In contrast to the competition assay, which is designed to measure $\mathrm{k}_{\mathrm{a}^{\prime}}$ a capacity assay measures the number of radicals that can be scavenged by an antioxidant (compare figure 7 and 11). Several antioxidant capacity assays have been developed. Ingold, Burton and Barclay were the first to develop this concept with the total radical-trapping antioxidant parameter (TRAP) [21]. In the TRAP assay, lipid peroxidation is measured by the rate of oxygen consumption. The antioxidant prevents the lipid peroxidation until the antioxidant is completely consumed. This induces a lag time in lipid peroxidation. The lag time is calibrated with trolox, which has a capacity of two, i.e. one molecule 
of trolox scavenges two radicals. The number of radicals that can be scavenged by the antioxidant is calculated using this calibration. This assay determines the capacity of an antioxidant. The rate of the reaction of the antioxidant with the reactive species is of minor importance, in contrast to competition assays. The problem addressed in the present paper does not apply for capacity assays. However, it has to be taken into account that the outcome of capacity assays also dependents on the assay conditions [22].

Since the introduction of the TRAP assay, other antioxidant capacity assays have been developed, for example the ORAC assay [23-25] and the TEAC assay [26-28]. In essence, these assays also determine the capacity of an antioxidant, i.e. the amount of reactive species the antioxidant can scavenge. These types of assays have their own pitfalls, but fall outside the scope of the present paper, which is restricted to competition assays.

In a competition assay, it has to be realized that during the assay the concentration of the antioxidant as well as that of the detector can be reduced to a considerable extent. The consumption of A and $D$ during the experiment as an inevitable consequence of the competition that has to take place, is a potential cause for inaccurate results.

Especially if $[R]_{0}$ is on the same order of magnitude as the initial concentration of detector or the observed $\mathrm{EC}_{50^{\prime}}$ the assay needs to be evaluated critically. In a competition assay, the reaction rate constant of the detector and that of the antioxidant should be comparable $\left(k_{d} \approx k_{a}\right)$ and the initial concentration of reactive species $\left([R]_{0}\right)$ has to be as low as possible to accurately assess antioxidant activity. 


\section{References}

1. Halliwell, B.; Gutteridge, J. M. C. Free radicals in biology and medicine. Oxford: Oxford University Press; 1999.

2. Website of The Radiation Chemistry Data Centre of the Notre Dame Radiation Laboratoy: http://www.rcdc. nd.edu.

3. Peskin, A. V.; Midwinter, R. G.; Harwood, D. T.; Winterbourn, C. C. Chlorine transfer between glycine, taurine, and histamine: reaction rates and impact on cellular reactivity. Free Radic. Biol. Med. 37:1622-1630; 2004.

4. Peskin, A. V.; Winterbourn, C. C. Kinetics of the reactions of hypochlorous acid and amino acid chloramines with thiols, methionine, and ascorbate. Free Radic. Biol. Med. 30:572-579; 2001.

5. Peskin, A. V.; Winterbourn, C. C. Taurine chloramine is more selective than hypochlorous acid at targeting critical cysteines and inactivating creatine kinase and glyceraldehyde-3-phosphate dehydrogenase. Free Radic. Biol. Med. 40:45-53; 2006.

6. Arts, M. J. T. J.; Haenen, G. R. M. M.; Voss, H. P.; Bast, A. Antioxidant capacity of reaction products limits the applicability of the Trolox Equivalent Antioxidant Capacity (TEAC) assay. Food Chem. Toxicol. 42:45-49; 2004.

7. Kooy, N.W.; Royall, J. A.; Ischiropoulos, H.; Beckman, J. S. Peroxynitrite-mediated oxidation of dihydrorhodamine 123. Free Radic. Biol. Med. 16:149-156; 1994.

8. Choi, E. H.; Chang, H. J.; Cho, J. Y.; Chun, H. S. Cytoprotective effect of anthocyanins against doxorubicininduced toxicity in $\mathrm{H} 9 \mathrm{C} 2$ cardiomyocytes in relation to their antioxidant activities. Food Chem. Toxicol. 45:1873-1881; 2007.

9. Gulcin, l.; Elmastas, M.; Aboul-Enein, H. Y. Determination of antioxidant and radical scavenging activity of Basil (Ocimum basilicum L. Family Lamiaceae) assayed by different methodologies. Phytother. Res. 21:354-361; 2007.

10. Zubia, M.; Robledo, D.; Freile-Pelegrin, Y. Antioxidant activities in tropical marine macroalgae from the Yucatan Peninsula, Mexico. J. Appl. Phycol. 19:449-458; 2007.

11. Renuka Devi, R.; Arumughan, C. Antiradical efficacy of phytochemical extracts from defatted rice bran. Food Chem. Toxicol. 45:2014-2021; 2007.

12. Huang, Z.; Guo, B. J.; Wong, R. N. S.; Jiang, Y. Characterization and antioxidant activity of selenium-containing phycocyanin isolated from Spirulina platensis. Food Chem. 100:1137-1143; 2007.

13. Halliwell, B.; Gutteridge, J. M.; Aruoma, O. I. The deoxyribose method: a simple "test-tube" assay for determination of rate constants for reactions of hydroxyl radicals. Anal. Biochem. 165:215-219; 1987.

14. Haenen, G. R. M. M.; De Rooij, B. M.; Vermeulen, N. P.; Bast, A. Mechanism of the reaction of ebselen with endogenous thiols: dihydrolipoate is a better cofactor than glutathione in the peroxidase activity of ebselen. Mol. Pharmacol. 37:412-422; 1990.

15. O’Dowd, Y.; Driss, F.; Dang, P. M.; Elbim, C.; Gougerot-Pocidalo, M. A.; Pasquier, C.; El-Benna, J. Antioxidant effect of hydroxytyrosol, a polyphenol from olive oil: scavenging of hydrogen peroxide but not superoxide anion produced by human neutrophils. Biochem. Pharmacol. 68:2003-2008; 2004.

16. Rietjens, S. J; Bast, A.; Haenen, G. R. M. M. New insights into controversies on the antioxidant potential of the olive oil antioxidant hydroxytyrosol. J. Agric. Food Chem. 55:7609-7614; 2007.

17. Pazdzioch-Czochra, M.; Widenska, A. Spectrofluorimetric determination of hydrogen peroxide scavenging activity. Anal. Chim. Acta 452:177-184; 2002.

18. Magalhaes, L. M.; Segundo, M. A.; Reis, S.; Lima, J. L. Methodological aspects about in vitro evaluation of antioxidant properties. Anal. Chim. Acta 613:1-19; 2008.

19. Long, L. H.; Clement, M. V.; Halliwell, B. Artifacts in Cell Culture: Rapid Generation of Hydrogen Peroxide on Addition of (-)-Epigallocatechin, (-)-Epigallocatechin Gallate, (+)-Catechin, and Quercetin to Commonly Used Cell Culture Media. Biochem. Biophys. Res. Commun. 273:50-53; 2000.

20. Esterbauer, H.; Gebicki, J.; Puhl, H.; Jurgens, G. The role of lipid peroxidation and antioxidants in oxidative modification of LDL. Free Radic. Biol. Med. 13:341-390; 1992. 
21. Barclay, L. R. C.; Locke, S. J.; MacNeil, J. M.; VanKessel, J.; Burton, G. W.; Ingold, K. U. Autoxidation of micelles and model membranes. Quantitative kinetic measurements can be made by using either water-soluble or lipid-soluble initiators with water-soluble or lipid-soluble chain-breaking antioxidants. J. Am. Chem. Soc. 106:2479-2481; 1984.

22. Wayner, D. D.; Burton, G. W.; Ingold, K. U. The antioxidant efficiency of vitamin C is concentration-dependent. Biochim. Biophys. Acta 884:119-123; 1986.

23. Cao, G.; Alessio, H. M.; Cutler, R. G. Oxygen-radical absorbance capacity assay for antioxidants. Free Radic. Biol. Med. 14:303-311; 1993.

24. Huang, D.; Ou, B.; Hampsch-Woodill, M.; Flanagan, J. A.; Prior, R. L. High-throughput assay of oxygen radical absorbance capacity (ORAC) using a multichannel liquid handling system coupled with a microplate fluorescence reader in 96-well format. J. Agric. Food Chem. 50:4437-4444; 2002.

25. Lopez-Alarcon, C.; Lissi, E. A novel and simple ORAC methodology based on the interaction of Pyrogallol Red with peroxyl radicals. Free Radic. Res. 40:979-985; 2006.

26. Miller, N. J.; Rice-Evans, C.; Davies, M. J.; Gopinathan, V.; Milner, A. A novel method for measuring antioxidant capacity and its application to monitoring the antioxidant status in premature neonates. Clin. Sci. (Lond.) 84:407-412; 1993

27. Re, R.; Pellegrini, N.; Proteggente, A.; Pannala, A.; Yang, M.; Rice-Evans, C. Antioxidant activity applying an improved ABTS radical cation decolorization assay. Free Radic. Biol. Med. 26:1231-1237; 1999.

28. van den Berg, R.; Haenen, G. R. M. M.; van den Berg, H.; Bast, A. Applicability of an improved Trolox equivalent antioxidant capacity (TEAC) assay for evaluation of antioxidant capacity measurements of mixtures. Food Chem. 66:511-517; 1999.

29. Yan, L. J.; Traber, M. G.; Kobuchi, H.; Matsugo, S.; Tritschler, H. J.; Packer, L. Efficacy of hypochlorous acid scavengers in the prevention of protein carbonyl formation. Arch. Biochem. Biophys. 327:330-334; 1996.

30. Floriano-Sanchez, E.; Villanueva, C.; Medina-Campos, O. N.; Rocha, D.; Sanchez-Gonzalez, D. J.; CardenasRodriguez, N.; Pedraza-Chaverri, J. Nordihydroguaiaretic acid is a potent in vitro scavenger of peroxynitrite, singlet oxygen, hydroxyl radical, superoxide anion and hypochlorous acid and prevents in vivo ozoneinduced tyrosine nitration in lungs. Free Radic. Res. 40:523-533; 2006.

31. Haenen, G. R. M. M.; Bast, A. Scavenging of hypochlorous acid by lipoic acid. Biochem. Pharmacol. 42:22442246; 1991.

32. Soobrattee, M. A.; Neergheen, V. S.; Luximon-Ramma, A.; Aruoma, O. I.; Bahorun, T. Phenolics as potential antioxidant therapeutic agents: mechanism and actions. Mutat. Res. 579:200-213; 2005.

33. Costa, D.; Marques, A. P.; Reis, R. L.; Lima, J. L.; Fernandes, E. Inhibition of human neutrophil oxidative burst by pyrazolone derivatives. Free Radic. Biol. Med. 40:632-640; 2006.

34. Gomes, A.; Fernandes, E.; Silva, A. M.; Santos, C. M.; Pinto, D. C.; Cavaleiro, J. A.; Lima, J. L. 2-Styrylchromones: novel strong scavengers of reactive oxygen and nitrogen species. Bioorg. Med. Chem. 15:6027-6036; 2007.

35. Ketsawatsakul, U.; Whiteman, M.; Halliwell, B. A reevaluation of the peroxynitrite scavenging activity of some dietary phenolics. Biochem. Biophys. Res. Commun. 279:692-699; 2000.

36. Pannala, A. S.; Razaq, R.; Halliwell, B.; Singh, S.; Rice-Evans, C. A. Inhibition of peroxynitrite dependent tyrosine nitration by hydroxycinnamates: nitration or electron donation? Free Radic. Biol. Med. 24:594-606; 1998.

37. Whiteman, M.; Ketsawatsakul, U.; Halliwell, B. A reassessment of the peroxynitrite scavenging activity of uric acid. Ann. N. Y. Acad. Sci. 962:242-259; 2002.

38. Heijnen, C. G. M.; Haenen, G. R. M. M.; Vekemans, J. A. J. M.; Bast, A. Peroxynitrite scavenging of flavonoids: structure activity relationship. Environ. Toxicol. Pharmacol. 10:199-206; 2001.

39. Paquay, J. B.; Haenen, G. R. M. M.; Stender, G.; Wiseman, S. A.; Tijburg, L. B.; Bast, A. Protection against nitric oxide toxicity by tea. J. Agric. Food Chem. 48:5768-5772; 2000

40. Rezk, B. M.; Haenen, G. R. M. M.; van der Vijgh, W. J. F.; Bast, A. The antioxidant activity of phloretin: the disclosure of a new antioxidant pharmacophore in flavonoids. Biochem. Biophys. Res. Commun. 295:9-13; 2002. 
41. Rezk, B. M.; Haenen, G. R. M. M.; van der Vijgh, W. J. F.; Bast, A. Tetrahydrofolate and 5-methyltetrahydrofolate are folates with high antioxidant activity. Identification of the antioxidant pharmacophore. FEBS Lett. 555:601-605; 2003.

42. Haenen, G. R. M. M.; Paquay, J. B. G.; Korthouwer, R. E. M.; Bast, A. Peroxynitrite Scavenging by Flavonoids. Biochem. Biophys. Res. Commun. 236:591-593; 1997.

43. Wang, L.; Tu, Y. C.; Lian, T. W.; Hung, J. T.; Yen, J. H.; Wu, M. J. Distinctive antioxidant and antiinflammatory effects of flavonols. J. Agric. Food Chem. 54:9798-9804; 2006.

44. Wada, M.; Kido, H.; Ohyama, K.; Ichibangase, T.; Kishikawa, N.; Ohba, Y.; Nakashima, M. N.; Kuroda, N.; Nakashima, K. Chemiluminescent screening of quenching effects of natural colorants against reactive oxygen species: Evaluation of grape seed, monascus, gardenia and red radish extracts as multi-functional food additives. Food Chem. 101:980-986; 2007.

45. Santocono, M.; Zurria, M.; Berrettini, M.; Fedeli, D.; Falcioni, G. Influence of astaxanthin, zeaxanthin and lutein on DNA damage and repair in UVA-irradiated cells. J. Photochem. Photobiol. B. 85:205-215; 2006.

46. Janisch, K. M.; Olschlager, C.; Treutter, D.; Elstner, E. F. Simulated digestion of Vitis vinifera seed powder: polyphenolic content and antioxidant properties. J. Agric. Food Chem. 54:4839-4848; 2006.

47. Sheean, P. D.;Hodges, L. D.; Kalafatis, N.;Wright, P.F. A.;Wynne, P.M.; Whitehouse, M.W.; Macrides, T. A. Bioactivity of extracts from gonadal tissue of the edible Australian purple sea urchin Heliocidaris erythrogramma. J. Sci. Food Agric. 87:694-701; 2007

48. Costa, D.; Moutinho, L.; Lima, J. L.; Fernandes, E. Antioxidant activity and inhibition of human neutrophil oxidative burst mediated by arylpropionic acid non-steroidal anti-inflammatory drugs. Biol. Pharm. Bull. 29:1659-1670; 2006.

49. Singh, B. N.; Singh, B. R.; Singh, R. L.; Prakash, D.; Sarma, B. K.; Singh, H. B. Antioxidant and anti-quorum sensing activities of green pod of Acacia nilotica L. Food Chem. Toxicol. 47:778-786; 2009.

50. Tai, C.; Gu, X.; Zou, H.; Guo, Q. A new simple and sensitive fluorometric method for the determination of hydroxyl radical and its application. Talanta 58:661-667; 2002.

51. Louit, G.; Hanedanian, M.; Taran, F.; Coffigny, H.; Renault, J. P.; Pin, S. Determination of hydroxyl rate constants by a high-throughput fluorimetric assay: towards a unified reactivity scale for antioxidants. Analyst 134:250255; 2009.

52. Van Noorden, C. J.; Butcher, R. G. The involvement of superoxide anions in the nitro blue tetrazolium chloride reduction mediated by NADH and phenazine methosulfate. Anal. Biochem. 176:170-174; 1989. 

$d x_{1}=-k_{j} x_{1}$
$d x_{2}=k_{2} x_{1}$ 


\section{Chapter 3}

\section{Prevention of a systematic underestimation of antioxidant activity in competition assays.}

The impact of unspecific reactions of the reactive species. 


\section{Abstract}

In antioxidant competition assays, an antioxidant (A) and a detector compound (D) compete for a reactive species $(R)$. In the evaluation of these assays, it is tacitly assumed that all of $R$ is captured by either $D$ or $A$. Due to the - by definition - high reactivity of $R$, unspecific reactions of $R$ are likely to occur and neglecting these reactions will result in a systematic underestimation of antioxidant activity. It was shown that in the standard hydroxyl radical scavenging assay this was indeed the case; the inaccurate mathematical evaluation resulted in an underestimation of antioxidant activity of $25 \%$ in this competition assay. The systematic underestimation of antioxidant activity can be prevented by using an adjusted Stern-Volmer equation that takes into account that only part of $\mathrm{R}$ is captured by D or A. 


\section{Introduction}

Free radicals and other reactive species have been implicated in many diseases. This forms the fundament of the health benefits ascribed to scavenging antioxidants. In this concept, the reactive species reacts with a specific biotarget, e.g. DNA, which is a key process in the etiology of a disease, e.g. cancer. Antioxidants compete with the biotarget for the reactive species and protect by scavenging the reactive species before it damages the biotarget.

Usually, the scavenging activity of antioxidants is measured in vitro in competition assays. In these assays the in vivo situation is mimicked in a test tube. A detector molecule (D), which stands for the biotarget, reacts readily with the reactive species $(R)$ with a known reaction rate constant $\left(k_{d}\right)$. A signal $(S)$, proportional to the amount of $D$ that has reacted with $R$, is measured. The potency to reduce $S$ reflects the activity of the antioxidant $(A)$.

In determining the activity of $A$, it is tacitly assumed that all of $R$ is captured by either $D$ or $A$. However, due to the - by definition - high reactivity of $R$ unspecific, parallel reactions of $R$ will occur. Neglecting these parallel reactions will lead to a systematic underestimation of antioxidant activity. This prompted us to study the impact of this error.

The most cited antioxidant competition assay is the hydroxyl radical assay described by Halliwell et al. [1] and therefore this assay was chosen in the present study. Antioxidant activity is expressed as the second order rate constant $\left(k_{a}\right)$ of the reaction of $A$ with $\mathrm{R}$. To obtain $k_{a^{\prime}}$ a Stern-Volmer equation is applied [1]. Also in other competition assays, the same [2] or similar [3] procedures are used. Our evaluation demonstrates that ignoring the unspecific, parallel reactions of $\mathrm{R}$ will lead to a substantial underestimation of antioxidant activity. This can be corrected by modifying the evaluation procedure.

\section{Material and Methods}

Chemicals

2-Deoxy-D-ribose (deoxyribose), ferric chloride, ethylenediaminetetraacetic acid, mannitol, 2-thiobarbituric acid were purchased from Sigma (St. Louis, MO, USA). Hydrogen peroxide was obtained from Riedel-deHaën (Seelze, Germany). Trichloroacetic acid was obtained from Serva (Heidelberg, Germany). Ethanol was purchased from Biosolve (Valkenswaard, The Netherlands). Ascorbic acid was obtained from Merck (Darmstadt, Germany).

\section{Competition assay for hydroxyl radicals using deoxyribose as detector}

The hydroxyl radical scavenging assay was performed according to Halliwell et al. [1]. The incubation mixture contained a fixed concentration of the detector molecule ( $D$, deoxyribose) and a variable concentration of the antioxidant ( $A$, ethanol). A fixed amount of reactive species ( $R$, hydroxyl radicals) is generated in the solution by the Fenton reaction. When indicated, $10 \mathrm{mM}$ mannitol was also present in the incubation mixture. Deoxyribose is degraded upon exposure to $\mathrm{OH}$. After an incubation of one hour, the reaction mixture is heated under acidic conditions, MDA (malondialdehyde) is formed and this is detected by its ability to react with 2-thiobarbituric acid to form a pink chromogen. This is quantified by measuring the absorbance at $532 \mathrm{~nm}$, giving the signal (S). One of the reasons 
for selecting ethanol and mannitol was that they did not show any of the reported deviations of competition assays [2-4].

The unadjusted Stern-Volmer equation

The original Stern-Volmer equation describes the kinetics of intermolecular photophysical deactivation. It can also be applied in the evaluation of competition assays [2] since the mathematics behind this is identical to photophysical deactivation.

$S=\frac{k_{d}[D]}{k_{d}[D]+k_{a}[A]} S_{A=0}$

Equation 1

A linear function is obtained when $1 / \mathrm{S}$ is plotted against $[\mathrm{A}]$.

$\frac{1}{S}=\frac{k_{o}}{k_{d}[D] S_{A=0}}[A]+\frac{1}{S_{A=0}}$

Equation 2

In these two equations $S_{A=0}$ is the signal, $S$, obtained without $A$ in the incubation mixture. From both functions $k_{a}$ can be obtained. A similar approach to determine $k_{a}$ that is also often applied and makes use of the same assumption that all of $R$ reacts with either $A$ or $D$, is:

$k_{a}=\frac{k_{d}[D]}{E C_{50}}$

Equation 3

The $\mathrm{EC}_{50}$ is the concentration of $\mathrm{A}$ that reduces $\mathrm{S}$ by half.

The adjusted Stern-Volmer equation

The adjusted Stern-Volmer equation takes parallel reactions of $\mathrm{R}$, other than those with D or A, into account. When these parallel reactions are first order in respect to $R$, it is not necessary to know the exact reactions that take place and an apparent reaction rate constant $\left(\mathrm{k}_{\mathrm{x}}\right)$ can be used. One of the parallel reactions in the evaluated assay will be the reaction of ${ }^{\circ} \mathrm{OH}$ with ascorbic acid that is present in the incubation mixture.

The apparent first order reaction rate constant, $k_{x^{\prime}}$ of the parallel reactions is determined in an experiment without $A$ and a variable concentration of $D$. The dependence of $S$ on $D$ is:

$S=\frac{k_{d}[D]}{k_{d}[D]+k_{x}} S_{\max }$

Equation 4

A linear function is obtained when $1 / S$ is plotted against $1 /[D]$.

$\frac{1}{S}=\frac{1}{S_{\max }}+\frac{k_{x}}{k_{d} S_{\max }} \frac{1}{[D]}$

Equation 5

In these two equations $S_{\max }$ is $S$ obtained from the fit when $[D]$ is infinitive or $1 /[D]=0 ; S_{\max }$ is $S$ when all of $R$ is captured by $D$. From both equations $k_{x}$ can be obtained. The value of $k_{x}$ is needed to 
determine $\mathrm{k}_{\mathrm{a}}$ with the adjusted Stern-Volmer equation:

$$
S=\frac{k_{d}[D]}{k_{d}[D]+k_{a}[A]+k_{x}} S_{\text {max }}
$$

Equation 6

A linear function of the adjusted Stern-Volmer equation is obtained when $1 / \mathrm{S}$ is plotted against [A].

$$
\frac{1}{S}=\frac{k_{a}}{k_{d}[D] S_{\max }}[A]+\frac{k_{d}[D]+k_{x}}{k_{d}[D] S_{\max }}
$$

From the linear fit that is obtained when $1 / \mathrm{S}$ is plotted against $[A]$, the $y$-axis intercept (b) and the slope (a) can be determined. The value of $k_{a}$ can be calculated according to $k_{a}=(a / b)^{*}\left(k_{d}[D]+k_{x}\right)$. If $k_{d}$ and $k_{x}$ are not known, the systematic underestimation in a competition assay can be determined quickly from $S_{\max }$ and $S$ in the assay without $A, S_{A=0^{\prime}}$ since the underestimation is equal to $\left(S_{\max }-S_{A=0}\right) /$ $\mathrm{S}_{\max } * 100 \%$.
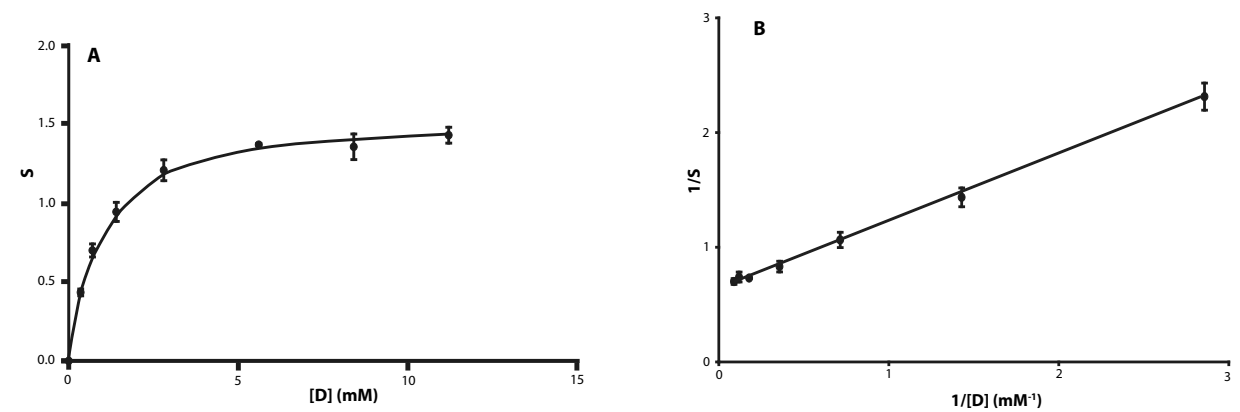

Figure 1. Determination of the rate constant of the parallel reaction $\left(k_{x}\right)$ in the $\cdot \mathrm{OH}$ scavenging assay. Hydroxyl radicals were generated by the Fenton reaction. No antioxidant was present in the incubation mixture in this experiment. Data are depicted as average $\pm S D(n=3)$. In panel A the signal, $S$ (the absorption at $532 \mathrm{~nm}$ due to the formation of MDA by the reaction of deoxyribose with $\cdot \mathrm{OH})$ is plotted against the concentration of detector $(D$, deoxyribose). In panel $B$ the double reciprocal plot of $S$ versus the concentration of $D$ is depicted. Both the linear and the nonlinear fit give $S_{\text {max }}=1.37$ and $k_{x}=2.64 * 10^{9} \mathrm{~s}^{-1}$.

\section{Results}

In the experiment depicted in figure 1, an increasing concentration of deoxyribose (D) was added to an $\cdot \mathrm{OH}(\mathrm{R})$ generating Fenton system. The absorbance (S) resulting from MDA formed by the reaction of deoxyribose with $\cdot \mathrm{OH}$, increased when the concentration of deoxyribose was increased. Even at the highest concentrations of deoxyribose tested, the fit of the curve indicates that signal (S) has not reached its maximum, despite the excess of deoxyribose (up to $12 \mathrm{mM}$ ) over the amount of $\cdot \mathrm{OH}$ formed $(0.06 \mathrm{mM}$, based on MDA formation at the highest concentration of deoxyribose tested). A sub-maximal $S$ at an excess of $D$ implies that only a part of $R$ was captured by $D$. This is indicative for the occurrence of parallel reactions of $R$, other than those of $R$ with $D$.

From the fit and the second order rate constant of deoxyribose with $\mathrm{OH}\left(\mathrm{k}_{\mathrm{d}}=3.1^{*} 10^{9} \mathrm{M}^{-1} \mathrm{~s}^{-1}\right)$, a first 
order rate constant of $2.64 \pm 0.16^{*} 10^{9} \mathrm{~s}^{-1}$ could be derived for all parallel reaction of $\mathrm{R}\left(\mathrm{k}_{\mathrm{x}}\right)$. This parallel reaction is actually the sum of all parallel, unspecific reactions of $\cdot \mathrm{OH}$ in the reaction mixture other than those with $D$.

Competition experiments for ethanol (A) using three concentrations of deoxyribose (D) were performed (figure 2). The $\mathrm{EC}_{50}$ 's obtained using 0.7, 2.8 and $7.0 \mathrm{mM}$ of deoxyribose were variable. The $\mathrm{k}_{\mathrm{a}}$ 's obtained for ethanol using the Stern-Volmer equation at 0.7, 2.8 and 7.0 mM of deoxyribose also appeared to be variable.

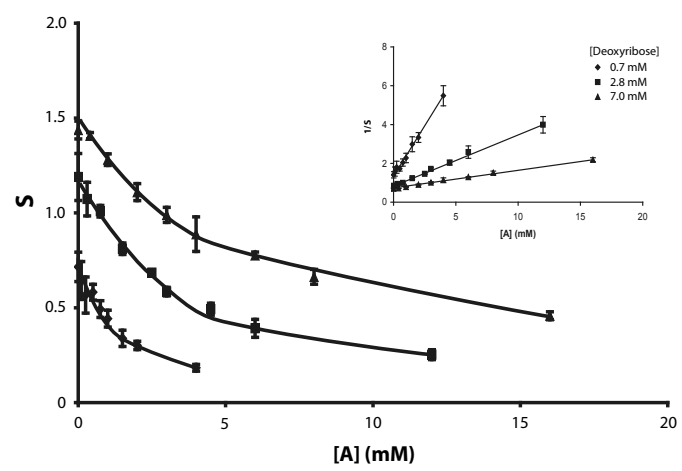

Figure 2. Determination of the $\mathrm{OH}$ scavenging activity $\left(k_{d}\right)$ of ethanol. The inhibition by ethanol $(A)$ of the signal $(S$, the absorbance at $532 \mathrm{~nm}$ ) is depicted. Data are depicted as average $\pm S D(n=3)$. In the insert the reciprocal value of $S$ is plotted on the $y$-axis. Second order rate constant calculated from the linear fits using the unadjusted Stern-Volmer relation yields $k_{a}(0.7 \mathrm{mM}$ deoxyribose $)=1.59 \pm 0.07^{*} 10^{9} \mathrm{M}^{-1} \mathrm{~s}^{-1}, \mathrm{k}_{a}(2.8 \mathrm{mM}$ deoxyribose $)=2.73 \pm 0.09^{*} 10^{9} \mathrm{M}^{-1} \mathrm{~s}^{-1}, \mathrm{k}_{a}(7.0 \mathrm{mM}$ deoxyribose $)=2.88 \pm 0.08^{*} 10^{9} \mathrm{M}^{-1} \mathrm{~s}^{-1}$. Second order rate constants calculated using the adjusted Stern-Volmer relation yield $k_{a}(0.7 \mathrm{mM}$ deoxyribose $)=3.1 \pm 0.3^{*} 10^{9} \mathrm{M}^{-1} \mathrm{~s}^{-1}, \mathrm{k}_{a}(2.8 \mathrm{mM}$ deoxyribose $)=3.6 \pm 0.2^{*} 10^{9} \mathrm{M}^{-1} \mathrm{~s}^{-1}, k_{a}(7.0 \mathrm{mM}$ deoxyribose $)=$ $3.6 \pm 0.1 * 10^{9} \mathrm{M}^{-1} \mathrm{~s}^{-1}$.

Using the adjusted Stern-Volmer equation, that takes the parallel reactions of $\mathrm{R}$ into account, the values for $\mathrm{k}_{\mathrm{a}}$ for ethanol obtained with 0.7,2.8 and 7.0 mM deoxyribose concentrations did not differ significantly.

To further examine the parallel reactions, an extra parallel reaction for $\mathrm{R}$ was introduced in the assay by adding a fixed concentration of mannitol (i.e. $10 \mathrm{mM}$ ) to the incubation mixture. Mannitol reacts relatively fast with $\cdot \mathrm{OH}\left(\mathrm{k}_{\mathrm{a}}=1.0^{*} 10^{9}\right.$ to $\left.1.8^{*} 10^{9} \mathrm{M}^{-1} \mathrm{~s}^{-1}[5]\right)$. It was found that the addition of 10 $\mathrm{mM}$ mannitol increased the reaction rate constant of the parallel reactions of $\mathrm{R}\left(\mathrm{k}_{\mathrm{x}}\right)$ (including the reaction of $\cdot \mathrm{OH}$ with mannitol) from $2.64^{*} 10^{9} \mathrm{~s}^{-1}$ to $29.3^{*} 10^{9} \mathrm{~s}^{-1}$ (figure 3 ).

The antioxidant activity of ethanol was also determined in the competition assay with the extra parallel reaction. The $k_{a}$ of ethanol obtained using the Stern-Volmer equation was greatly reduced by the presence of mannitol, i.e. from $2.7^{*} 10^{9} \mathrm{M}^{-1} \mathrm{~s}^{-1}$ to $0.8^{*} 10^{9} \mathrm{M}^{-1} \mathrm{~s}^{-1}$ (figure 4). Applying the adjusted Stern-Volmer equation, however, yields the same $k_{a}$ of ethanol, i.e. $k_{a}(0 \mathrm{mM}$ mannitol $)=k_{a}(10 \mathrm{mM}$ mannitol) $=3.6^{*} 10^{9} \mathrm{M}^{-1} \mathrm{~s}^{-1}$. 

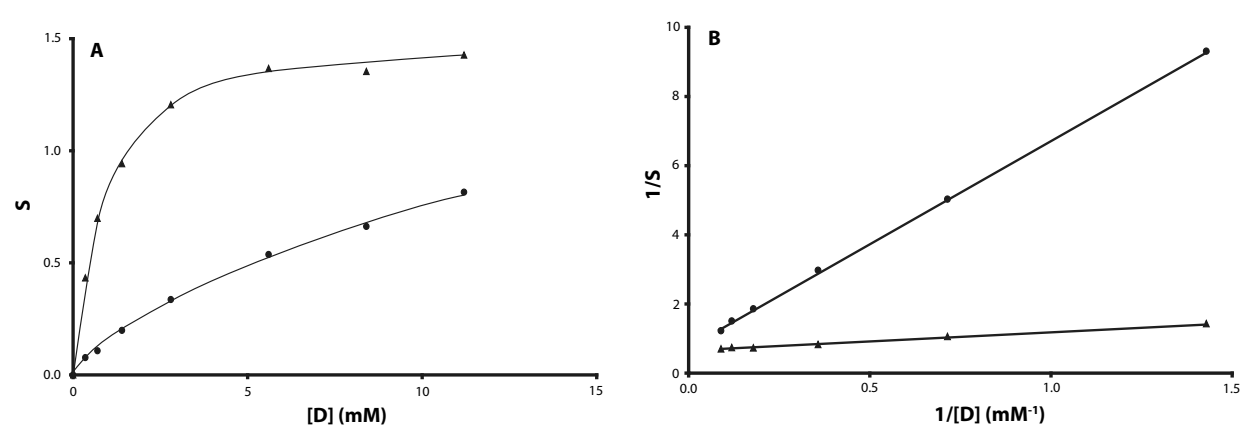

Figure 3. Increasing the reaction rate of the parallel reactions $(k)$ by mannitol. A fixed concentration of mannitol (10 $m M)$ was added to the reaction system to increase $k_{x}$. Panel A shows the plot of the signal ( $S$, the absorbance at 532 $n m$ ), versus the concentration of the detector ( $D$, deoxyribose) without (triangles) or with $10 \mathrm{mM}$ mannitol (circles). In Panel $B$, the double reciprocal plot of $S$ versus the concentration of $D$ is depicted. Both the linear and the nonlinear fit give first order reaction rates for the parallel reactions, $k_{x}$ (no mannitol) $=2.78^{*} 10^{9} \mathrm{~s}^{-1}, k_{x}\left(10 \mathrm{mM}\right.$ mannitol) $=29.3^{*} 10^{9} \mathrm{~s}^{-1}$.

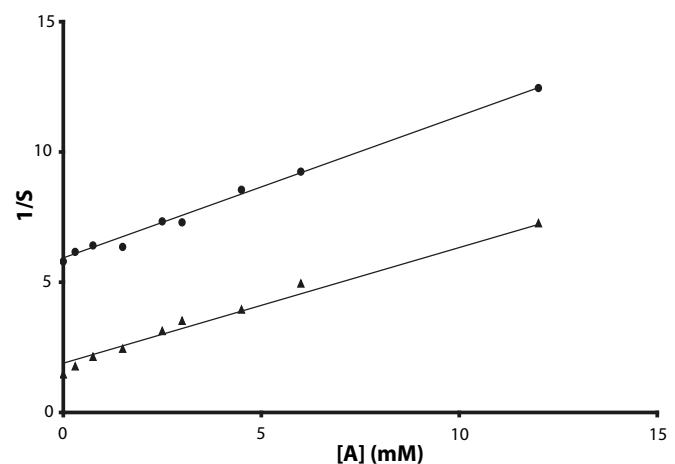

Figure 4. Effect of increasing the reaction rate of the parallel reaction ( $k_{x}$ ) on the $\cdot \mathrm{OH}$ scavenging activity ( $k_{d}$ ) of ethanol. Adding mannitol (10 mM) increased $k_{x}$ from $2.78^{*} 10^{9} \mathrm{~s}^{-1}$ (no mannitol, triangles) to $29.3^{*} 10^{9} \mathrm{~s}^{-1}$ (10 mM mannitol, circles). In the figure the double reciprocal plot of $S$ is plotted versus the concentration of the antioxidant ( $A$, ethanol) is depicted. Second order rate constants for scavenging ${ }^{\cdot} \mathrm{OH}$ by ethanol calculated using the unadjusted Stern-Volmer relation are $k_{a}$ (no mannitol) $=2.7^{*} 10^{9} \mathrm{M}^{-1} \mathrm{~s}^{-1}$ and $\mathrm{k}_{a}\left(10 \mathrm{mM}\right.$ mannitol) $=0.8^{*} 10^{9} \mathrm{M}^{-1} \mathrm{~s}^{-1}$. Second order rate constants calculated using the adjusted Stern-Volmer relation are $k_{a}($ no mannitol $)=3.6^{*} 10^{9} \mathrm{M}^{-1} \mathrm{~s}^{-1}$ and $\mathrm{k}_{a}(10 \mathrm{mM}$ mannitol $)=3.6^{*} 10^{9} \mathrm{M}^{-1} \mathrm{~s}^{-1}$.

\section{Discussion}

The principle of a competition assay is that an antioxidant, A, has to compete with a detector molecule, D, for a reactive species, R. Our evaluation shows that in a competition assay A has to compete not only with $D$, but also with the parallel, unspecific reactions of $R$. These parallel reactions are incorrectly neglected in the common evaluation procedures, e.g. those using the unadjusted Stern-Volmer equation. This is schematically depicted in figure 5. The underestimation of antioxidant activity resulting from the use of the unadjusted Stern-Volmer equation is equal to the fraction of $R$ not captured by $D$ in the absence of $A$. 


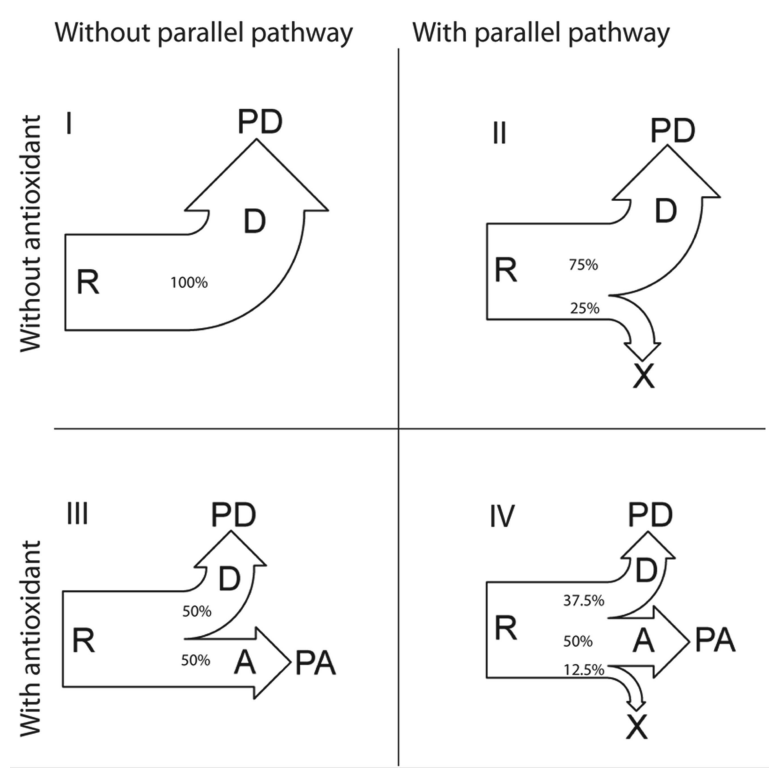

Figure 5. Schematic visualization of the reactions in a competition assay. The compounds present at the start of the assay are the reactive species, $R$; the detector molecule, $D$; and the antioxidant, $A$. The product formed in the reaction of $R$ with $D$ is $P D$; the product formed in the reaction of $R$ with $A$ is $P A$. As shown in the present paper, also parallel reactions of $R$ other than those with $A$ and $D$ take place. The sum of all these parallel reactions leads to the formation of products $(X)$ that do not have to be identified. It was found that for the $\mathrm{OH}$ radical scavenging assay all parallel reactions are first order in respect to $R$. The relative importance of the reactions is given by the width of the arrow and depicted by the percentage. As much as possible, the data of the hydroxyl scavenging assay evaluated in the present paper are used in this visualization.

In the visualization, two different situations are combined, i.e. the situation with or without the parallel pathway and the situation with or without antioxidant (A) in the incubation system. In the situation with $A$, the concentration of $A$ was identical to the $E C_{50}$ value, i.e. the concentration of $A$ that reduced the formation of PD by $50 \%$.

Panel I, without antioxidant, parallel pathway not taken into account. It is assumed that $100 \%$ of $R$ is captured by D, but often this is not correct.

Panel II, without antioxidant, parallel pathway taken into account. A substantial part of R is not captured by D. For the hydroxyl radical scavenging assay using the conditions given in the paper of Halliwell et al. [1], this parallel pathway account for $25 \%$ of the total amount of $R$ and only $75 \%$ of $R$ reacts with D.

Panel III, with antioxidant, parallel pathway not taken into account. Often it is assumed that the antioxidant only competes with the detector for the reactive species. The amount of $R$ that reacts with $D$ is reduced from 100\% to $50 \%$ at the $E C_{50}$ value of $A$.

Panel IV, with antioxidant, parallel pathway taken into account. Due to the parallel pathway, D only captures part of $R$ (in the example depicted only 75\%) when $A$ is absent. This means that at a concentration of $A$ equal to the $E C_{50}$ value, only $37.5 \%$ of $R$ reacts with $D$. To reduce the reaction of $R$ with $D$ by half, $A$ also has to compete with the parallel pathway and $A$ has to capture $50 \%$ of $R$. It appears that for the hydroxyl radical scavenging assay using the conditions given in the paper of Halliwell et al. [1], the reaction rate of D with $R$ is $25 \%$ lower than the reaction of $A$ with $R$, when the concentration of $A$ is equal to the $E C_{50}$ value. The incorrect assumption that is often made is that, at the $E C_{50}$ value, the reaction rate of $A$ with $R$ equals that of $D$ with $R$. This mistake is responsible for the $25 \%$ systematic underestimation in antioxidant activity (expressed as the second order rate constant of the reaction of $A$ with $R, k_{d}$ ) observed in the evaluated assay. The adjusted Stern-Volmer equation takes the parallel pathway into account and prevents a systematic underestimation of $k_{a}$. 
There are two strategies to deal with the parallel pathway of R. The first strategy is to increase the detector concentration so that the parallel pathway is small enough to be neglected. To scavenge $99 \%$ of all of the $\mathrm{OH}$ formed in the standard assay, the concentration of deoxyribose has to increase considerably, i.e. from $2.8 \mathrm{mM}$ to $82 \mathrm{mM}$. Increasing the amount of detector means that the concentration of antioxidant has to increase proportionally to give substantial inhibition. The limited solubility of many antioxidants is a major impediment for this strategy. Therefore the strategy to increase the concentration of $D$ is often not suitable.

The alternative strategy is to take the parallel reactions into account in calculating the $k_{a}$ of the antioxidant and use the adjusted Stern-Volmer equation to calculate $k_{a}$. The present study shows the validity and ease of this strategy.

The use of the unadjusted Stern-Volmer assay will result in different values for $k_{a}$ of a certain antioxidant when different assay conditions are used. Using the unadjusted Stern-Volmer equation Wang et al. [6] reported a $\mathrm{k}_{\mathrm{a}}$ of mannitol, the reference $\mathrm{OH}$ scavenger, of $2.86^{*} 10^{9} \mathrm{M}^{-1} \mathrm{~s}^{-1}$ at a 60 $\mathrm{mM}$ concentration of deoxyribose. Using a deoxyribose concentration of $2.8 \mathrm{mM}$, we obtained a different $\mathrm{k}_{\mathrm{a}}$ of mannitol of $2.13^{*} 10^{9} \mathrm{M}^{-1} \mathrm{~s}^{-1}$ with the unadjusted Stern-Volmer equation. Applying the adjusted Stern-Volmer equation, the $k_{a}$ for mannitol obtained by us or Wang et al. [6] is practically identical, namely $2.84^{*} 10^{9} \mathrm{M}^{-1} \mathrm{~s}^{-1}$ and $2.89^{*} 10^{9} \mathrm{M}^{-1} \mathrm{~s}^{-1}$, respectively. This shows that seemingly different antioxidant activities can be corrected to correspond with each other by applying the adjusted Stern-Volmer equation. The validity of the adjusted Stern-Volmer equation was also demonstrated in the present study for ethanol in the competition assay with the extra parallel reaction and the competition assay using three different concentrations of detector.

Beside the concentration of detector, competition assays for ${ }^{\circ} \mathrm{OH}$ scavenging also differ in the way the $\mathrm{OH}$ radicals are formed (e.g. Fenton reactions [7,8], Fenton like reactions [9-11], pulse radiolysis [12-16], photo-Fenton systems (17], hypoxanthine/xanthine oxidase systems (18]). Also different detectors are used (e.g. deoxyribose [1], DMSO [19], DMPO [20], salicylic acid [21], luminol [22-25], coumarin [26-29]). In competition assays for reactive species other than the hydroxyl radical, again other experimental conditions are used.

Based on the big differences in experimental conditions, the parallel reactions of $\mathrm{R}$ in various competition assays, and thus the underestimation of the $k_{a}$ obtained, will greatly differ. The actual underestimation in a specific competition assays is difficult to estimate from literature data, because reactions of $\mathrm{R}$ other than those with $\mathrm{A}$ and $\mathrm{D}$ are ignored, and the dependence of $\mathrm{S}$ on the concentration of $D$ is hardly ever studied. In the rare cases that the relationship is displayed, the parallel pathway appears to be considerable (e.g. $>50 \%$ in the assay using salicylic acid as detector (21]), giving rise to a substantial systematic underestimation of antioxidant activity. This systematic underestimation is probably widespread and not acknowledged.

In vitro experiments with e.g. cells, and in vivo studies on scavenging antioxidants are in essence also competition assays. In these tests the biological target of the reactive species is equivalent to $D$ and the antioxidant competes with the biotarget for the reactive species. Similar to the competition 
assay in the test tube, in biological systems the antioxidant also has to compete with the unspecific, parallel reactions to scavenge a substantial part of the reactive species which is needed to provide some protection of the biotarget. Due to the high reactivity of the reactive species, many parallel and very fast reactions will occur in the biological system. For example, glucose reacts with hydroxyl radicals at a rate comparable to that of ethanol and glucose is present in biological systems in relatively high concentrations. The reaction with glucose will be one of the parallel reactions of the hydroxyl radial in vivo. Numerous other biological compounds that act as sacrificial antioxidant for the hydroxyl radical, will also react with the hydroxyl radical. No antioxidant in physiological achievable concentrations is able to compete efficiently with all of these parallel reactions of the hydroxyl radical. An antioxidant might not be able to offer adequate protection in cells or in vivo, despite a relatively high rate constant of its reaction with the reactive species (that might be even higher than that of the biotarget) because most of the reactive species react in parallel reactions as in the case of the hydroxyl radical. This shows the impact of the parallel reactions of the reactive species. It should be noted that our findings are not limited to the hydroxyl radical. Due to the intrinsic high reactivity of reactive species, parallel reactions need to be considered with any reactive species. The results obtained in the present study with the hydroxyl radical assay unambiguously demonstrate that the mathematical evaluation of data obtained in antioxidant competition assays has been inaccurate. A minor adjustment of the evaluation procedure prevents this inaccuracy. The scavenging of radicals by antioxidants does not only offer protection. The scavenging reaction generates oxidized and often very reactive products of the antioxidant, which are involved in antioxidant toxicity $[30,31]$. For a substantial physiological effect, it is not necessary that the antioxidant scavenges all the radicals. The second order rate constant is needed to quantify the potential effect of the antioxidant products. Also for this purpose, this rate constant has to be determined accurately.

In conclusion, our study shows that for accurate determination of the second rate constant of the reactive species with an antioxidant in a competition assay, the parallel, unspecific reactions of the reactive species (i.e. reactions of the reactive species other than those with the antioxidant or detector) should be taken into account, for example by using the adjusted Stern-Volmer equation. 


\section{References}

1. Halliwell, B., Gutteridge, J.M., Aruoma, O.I., The deoxyribose method: a simple "testtube" assay for determination of rate constants for reactions of hydroxyl radicals. Anal. Biochem.,1987. 165: p. 215-219.

2. Pino, E. and Lissi, E.A., Quantitative treatment of the kinetics of free- radical-mediated damage. Protection by free-radical scavengers. Helv. Chim. Acta, 2002. 84: p. 3677-3685.

3. Balk, J.M., Bast, A., Haenen, G.R.M.M., Evaluation of the accuracy of antioxidant competition assays: incorrect assumptions with major impact. Free Radic. Biol. Med., 2009. 47: p. 135-144.

4. Haenen, G.R.M.M., Arts, M.J.T.J., Bast, A., Coleman, M.D., Structure and activity in assessing antioxidant activity in vitro and in vivo: A critical appraisal illustrated with the flavonoids. Environ. Toxicol. Pharmacol., 2006. 21: p. 191-198.

5. Goldstein, S., Czapski, G., Mannitol as an $\mathrm{OH}^{\bullet}$ scavenger in aqueous solutions and in biological systems. Int. J. Radiat. Biol. Relat. Stud. Phys. Chem. Med., 1984. 46: p. 725-729.

6. Wang, Y.G., Zhu, F.R., Chen, J., Han, F.D., Wang, H.Y., Effects of Pro-Arg, a novel dipeptide derived from protamine hydrolysate on $\mathrm{H}_{2} \mathrm{O}_{2}$-induced oxidative stress in human diploid fibroblasts. Biol. Pharm. Bull., 2009. 32: p. 389-393.

7. Fenton, H.J.H., Oxidation of tartaric acid in the presence of iron. J. Chem. Soc. 1894, 65: p. 899- 910.

8. Haber, F., Weiss, J.J., The catalytic decomposition of hydrogen peroxide by iron salts. Proc. Roy. Soc. B-Biol. Sci., 1934. A147: p. 332-351.

9. Czapski, G., Samuni, A., Meisel, D., Reactions of organic radicals formed by some "Fenton-like" reagents, J. Phys., 1971. 75: p. 3271-3280.

10. Heckman, R.A., Espenson, J.H., Kinetics and mechanism of oxidation of cobalt(II) macrocycles by iodine, bromine, and hydrogen peroxide. Inorg. Chem., 1979. 18: p. 38-43.

11. Moorhouse, C.P., Halliwell, B., Grootveld, M., Gutteridge, J.M.C., Cobalt(II) ion as a promoter of hydroxyl radical and possible 'crypto-hydroxyl' radical formation under physiological conditions. Differential effects of hydroxyl radical scavengers. Biochim. Biophys. Acta, 1985. 843: p. 261-268.

12. Dorfman, L.M., Pulse radiolysis: fast reaction studies in radiation chemistry. Science, 1963. 141: p. 493 - 498.

13. Keene, J.P., Pulse radiolysis apparatus. J. Sci. Instr., 1964. 41: p. 493.

14. Dorfman, L.M., Matheson, M.S., Pulse radiolysis, in: G. Porter (Ed.) Progress in Reaction Kinetics III, Pergamon Press, London, 1965, pp. 237-301.

15. Matheson, M.S., Dorfman, L.M., Pulse Radiolysis. M.I.T. Press, Cambridge, Massachusetts, 1969.

16. Anbar, M., Neta, P., A compilation of specific bimolecular rate and hydroxyl radical with inorganic and organic compounds in aqueous solution. Int. J. App. Rad., 1967. 18: p. 493-523.

17. Tobin, D., Arvanitidis, M., Bisby, R.H., One-electron oxidation of "photo-Fenton" reagents and inhibition of lipid peroxidation. Biochem. Bioph. Res. Comm., 2002. 299: p. 155-159.

18. Yang, X.F., Guo, X.Q., Fe(II)-EDTA chelate-induced aromatic hydroxylation of terephthalate as a new method for the evaluation of hydroxyl radical-scavenging ability. Analyst, 2001. 126: p. 928-932.

19. Tai, C., Gu, X., Zou, H., Guo, Q., A new simple and sensitive fluorometric method for the determination of hydroxyl radical and its application. Talanta, 2002. 58: p. 661-667.

20. Calliste, C.A., Trouillas, P., Allais, D.P., Simon, A., Duroux, J.L., Free Radical Scavenging Activities Measured by Electron Spin Resonance Spectroscopy and B16 Cell Antiproliferative Behaviors of Seven Plants. J. Agr. Food. Chem., 2001. 49: p. 3321-3327.

21. Zhu, B.Z., Kitrossky, N., Chevion, M., Evidence for production of hydroxyl radicals by pentachlorophenol metabolites and hydrogen peroxide: A metal-independent organic fenton reaction. Biochem. Bioph. Res. Comm., 2000. 270: p. 942-946.

22. Hirayama, O., Yida, M., Evaluation of hydroxyl radical-scavenging ability by chemiluminescence. Anal. Biochem., 1997. 251: p. 297-299.

23. Oosthuizen, M.M.J., Greyling, D., Hydroxyl radical generation: the effect of bicarbonate, dioxygen and buffer concentration on pH-dependent chemiluminescence. Redox Rep., 2001. 6: p. 105-116. 
24. Yildiz, G., Demiryürek, A.T., Ferrous iron-induced luminol chemiluminescence: a method for hydroxyl radical study. J. Pharmacol. Toxicol., 1998. 39: p. 179-184.

25. Rao, P.S., Luber, J.M., Milinowicz, J., Lalezari, P., Mueller, H.S., Specificity of oxygen radical scavengers and assessment of free radical scavenger efficiency using luminol enhanced chemiluminescence. Biochem. Biophys. Res. Comm., 1988. 150: p. 39-44.

26. Louit, G., Hanedanian, M., Taran, F., Coffigny, H., Renault, J.P., Pin, S., Determination of hydroxyl rate constants by a high-throughput fluorimetric assay: towards a unified reactivity scale for antioxidants. Analyst. 2009. 134: p. 250-255.

27. Gopakumar, K., Kini, U.R., Ashawa, S.C., Bhandari, N.S., Krishnan, G.U., Krishnan, D., Gamma irradiation of coumarin in aqueous solution. Radiat. Eff. Defects, 1977. S 32: p. 199 - 203.

28. Ashawa, S.C., Kini, U.R., Madhvanath, U., The aqueous coumarin system as a low range chemical dosimeter. Int. J. App. Rad. Is., 1979. 30: p. 7-10.

29. Makrigiorgos, G.M., Baranowska-Kortylewicz, J., Bump, E., Sahu, S.K., Berman, R.M., Kassis, A.l., A method for detection of hydroxyl radicals in the vicinity of biomolecules using radiation-induced fluorescence of coumarin. Int. J. Radiat. Biol., 1993. 63: p. 445 - 458.

30. Bast, A., Haenen, G.R.M.M., Toxicity of antioxidants and their metabolites. Environment. Toxicol. Pharmacol., 2002. 11: p. $251-258$.

31. Boots, A.W., Li, H., Schins, R.P.F., Duffin, R., Heemskerk, J.W.M., Bast, A., Haenen, G.R.M.M. The quercetin paradox. Toxicol. Appl. Pharmacol., 2007. 222: p. 89 - 96. 

$d x_{1}=-k_{j} \cdot x_{1}$
$d x_{2}=k_{2} w_{1}$ 


\section{Chapter 4}

Stoichiometry and capacity versus reaction rate in antioxidant activity

Not the reaction rate, but the number of reactive species scavenged determines the observed antioxidant activity. 


\section{Abstract}

The activity of scavenging antioxidants is usually tested in competition assays, where an antioxidant has to compete with a detector molecule for a reactive species. The reaction rate constant of the scavenging reaction of the antioxidant with the reactive species $\left(k_{a}\right)$ is in general used to describe the antioxidant activity. Detailed evaluation of several hypochlorous acid (HOCl) competition assays revealed that the observed activity of an antioxidant is, however, not primarily governed by the $k_{a}$. The stoichiometry of the scavenging reaction and the reactivity of the products formed out of the antioxidant by the scavenging reaction are involved in the observed antioxidant activity. The contribution of the products of the antioxidant has to be included in the evaluation of antioxidant activity. The antioxidant activity of an antioxidant observed in a competition assay is best described by the observed capacity, i.e. the average number of reactive species scavenged by a single molecule of antioxidant, including that of the antioxidant products. 


\section{Introduction}

The onset of many diseases is related to the production of reactive species that damage essential cellular compounds. For example, excessive production of the reactive species hypochlorous acid $(\mathrm{HOCl})$ inactivates $\mathrm{a}_{1}$-antiprotease $\left(\mathrm{a}_{1}-\mathrm{AP}\right)$ which is key in the development of lung emphysema [1]. Antioxidants can protect the biological targets against the devastating effects of reactive species by scavenging the reactive species.

The antioxidant activity of compounds is frequently tested in competition assays. In these assays, the antioxidant has to protect a detector compound (D) by scavenging the reactive species (R). Various assays have been developed even for a single type of reactive species. $\mathrm{HOCl}$ scavenging of compounds can be tested in, for example, the acetylcholine esterase (AChE) assay [2], the albumin assay [3], the dihydrorhodamine assay (DHR123) [4], the thionitrobenzoic acid assay [5], and the $a_{1}$ antiprotease (a,-AP) assay [6], all are named after the detector used.

Although a competition assay is easy to perform, the interpretation is complex since the observed antioxidant activity is influenced by many factors. For example, the initial concentration of reactive species influences the observed activity of an antioxidant in a particular assay [7]. The deviation between the observed activity and theoretical activity can be estimated using figure 1. This figure reveals that in some $\mathrm{HOCl}$ scavenging competition experiments (such as glutathione in the AChE assay, table 1) products of the antioxidant contribute to the observed antioxidant activity. In other experiments (like glutathione in the methionine assay, table 1) the products of the antioxidant do not contribute to the observed antioxidant activity. This difference in the contribution of products between competition experiments prompted us to elucidate the contribution of these products to the observed antioxidant activity. Our conclusion is that products of the antioxidant formed during the scavenging reaction of the parent antioxidant, often have a very substantial contribution to the observed activity of an antioxidant.

Table 1: The antioxidant activity, given by the $E C_{50}$ of reduced glutathione (GSH), glutathione disulfide (GSSG) and lipoic acid (LA) measured in seven different competition assays. The assay is denoted by the detector used. The initial concentration of $\mathrm{HOCl}\left([\mathrm{HOCl}]_{0}\right)$ is also given. The concentrations are given in $\mu \mathrm{M}$.

\begin{tabular}{|c|c|c|c|c|c|c|c|}
\hline & GSH & & GSSG & & LA & & Ref \\
\hline Detector & $\mathrm{EC}_{50}$ & {$[\mathrm{HOCl}]_{0}$} & $\mathrm{EC}_{50}$ & {$[\mathrm{HOCl}]_{0}$} & $\mathrm{EC}_{50}$ & {$[\mathrm{HOCl}]_{0}$} & \\
\hline$a_{1}-A P$ & $20^{A}$ & 50 & $>100^{F}$ & 50 & $30^{\prime}$ & 50 & [6] \\
\hline MET & $520^{B}$ & 630 & n.d. & n.d. & $890^{\prime}$ & 630 & \\
\hline LA & $630^{c}$ & 630 & n.d. & n.d. & n.d. & n.d. & \\
\hline \multirow[t]{2}{*}{ DHR123 } & $1.3^{\mathrm{D}}$ & 5 & $9^{G}$ & 5 & $5^{K}$ & 5 & [14] \\
\hline & n.d. & n.d. & n.d. & n.d. & $2.4^{\mathrm{L}}$ & 5 & [15] \\
\hline AChE & 0.2 & 1 & 0.3 & 1 & 0.4 & 1 & [2] \\
\hline \multirow[t]{2}{*}{ Albumin } & $38^{\mathrm{E}}$ & 200 & $127^{\mathrm{H}}$ & 200 & $100^{M}$ & 200 & [16] \\
\hline & 200 & 1600 & n.d. & n.d. & 690 & 1600 & [3] \\
\hline Luminol & n.d. & n.d. & n.d. & n.d. & 6 & 25 & {$[17,18]$} \\
\hline
\end{tabular}

Superscript capital letters indicate the data in figure 1. n.d.: no data available. 


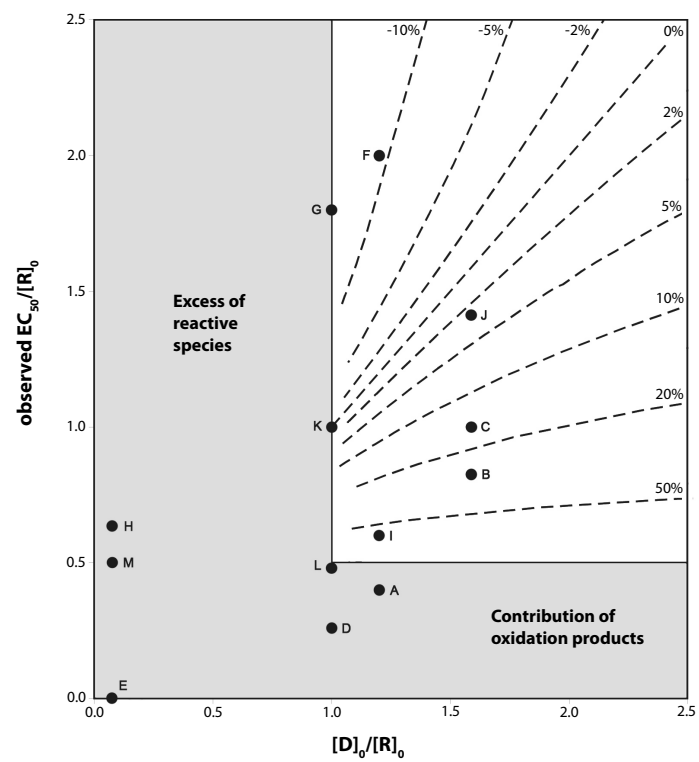

Figure 1. Estimation of the accuracy of the activity of several antioxidants obtained in various competition assays. Each point represents a specific antioxidant in a specific assay. The letter accompanying the point refers to the data presented in table 1. The ratio of the observed $E C_{50}$ and the initial concentration of reactive species, $[R]_{0}$ (both in molar) is plotted against the ratio of the initial concentration of detector, $[D]_{\sigma}$ and the initial amount of $[R]_{0}$ (both in molar). The dashed lines give the deviation of the observed $E C_{50}$ from the theoretical $E C_{50}[7]$. The grey area in the bottom indicates conditions that oxidation products of the antioxidant contribute to the assay.

\section{Materials}

Lipoic acid (LA) and $\beta$-lipoic acid ( $\beta$-LA) were obtained from Asta Medica AG (Frankfurt, Germany). Reduced glutathione (GSH), oxidized glutathione (GSSG), sodium hypochlorite (NaOCl), 5,5'-dithiobis(2-nitrobenzoic acid) (DTNB), L-methionine (MET) and 1,4-dithiotreitol (DTT) were purchased from Sigma-Aldrich (St. Louis, USA). All other chemicals were of analytical grade. All experiments were performed in $145 \mathrm{mM}$ potassium phosphate buffer $\mathrm{pH}$ 7.4.

\section{Methods}

Thionitrobenzoic acid (TNB) was prepared by incubating DTNB with sodium borohydride for 30 minutes at $37^{\circ} \mathrm{C}$. The concentration of TNB was determined spectrophotometrically at a wavelength of $412 \mathrm{~nm}\left(\varepsilon_{412}=13600 \mathrm{M}^{-1} \mathrm{~cm}^{-1}\right)$.

Stoichiometry and capacity: The stoichiometry of the antioxidant is defined as the number of molecules of reactive species that react directly with one molecule of antioxidant. The observed stoichiometry was quantified by determining the concentration of the antioxidant immediately (within 30 seconds) after addition of a relatively small amount of reactive species. The remaining concentration of the antioxidant was plotted against the initial concentration of reactive species 
and the data was fitted linearly. The intercept of the linear fit with the $x$-axis was divided by the initial concentration of antioxidant to obtain the observed stoichiometry. It should be noted that the observed stoichiometry, determined by this procedure, includes the products that react much faster with the reactive species than does the parent compound and might deviate from the actual stoichiometry.

The total scavenging capacity is defined as the number of molecules of the reactive species that are scavenged by the antioxidant within a certain time span, when an excess of reactive species is added to the antioxidant. The total scavenging capacity is the sum of the stoichiometry of the parent antioxidant, of the first product and of all other products formed in subsequent reactions. The total scavenging capacity of the antioxidants was determined by adding $\mathrm{HOCl}$ in a concentration range of 0-10 $\mathrm{mM}$ to a fixed concentration of the antioxidant of $1 \mathrm{mM}$. Thirty seconds after adding $\mathrm{HOCl}$, the concentration of remaining $\mathrm{HOCl}$ that was in excess, was determined spectrophotometrically. The remaining concentration of $\mathrm{HOCl}$ was plotted against the initial concentration of reactive species and the data was fitted linearly. The intercept with the $x$-axis was divided by the initial concentration of the antioxidant to obtain the total scavenging capacity. The procedure to determine the observed stoichiometry and total scavenging capacity of DTT is illustrated in the inset of figure 2 .

Competition assay: MET was used as detector in a final concentration of $1 \mathrm{mM}$. Different concentrations of the antioxidant (TNB or DTT) were added to the MET containing solution. The mixture of the detector and the antioxidant was pre-incubated at $37^{\circ} \mathrm{C}$ for 5 minutes. During rigorous mixing, $\mathrm{HOCl}$ was added (final concentration of $0.14 \mathrm{mM}, 0.35 \mathrm{mM}$ or $0.63 \mathrm{mM}$ ). After completion of the reaction, the remaining amount of the antioxidant was determined. To determine the remaining amount of DTT, the sample was diluted 50 times. DTNB was added (final concentration $0.2 \mathrm{mM}$ ) and the formed TNB was determined spectrophotometrically.

The $\mathrm{EC}_{50}$ was determined for each antioxidant and $\mathrm{HOCl}$ concentration used. The $\mathrm{EC}_{50}$ is defined as the concentration of antioxidant that reduces the signal produced by the detector by 50 percent compared to the signal obtained in the absence of the antioxidant.

The advantage of the $\mathrm{HOCl}$ scavenging assay is that all of the reactive species is scavenged by either the detector or antioxidant, and therefore there is no systematic underestimation of antioxidant activity as in numerous other assays (chapter 3 ). 


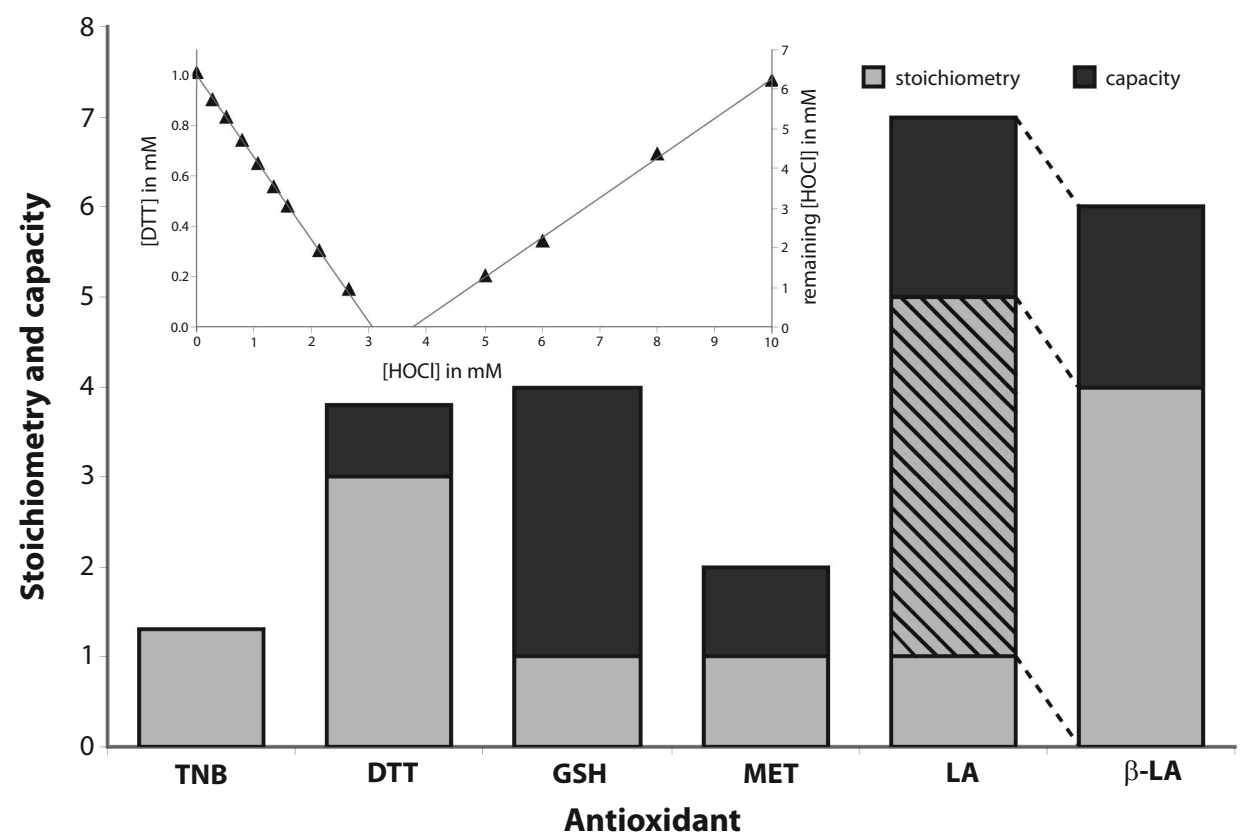

Figure 2. The observed stoichiometry and the total scavenging capacity of the reaction of six antioxidants with HOCl. The observed stoichiometry (given in grey) of the antioxidant reaction with $\mathrm{HOCl}$ is part of the total scavenging capacity (given by the height of the bar) of $\mathrm{HOCl}$ by the antioxidant. The observed stoichiometry of $\beta$-LA (the first oxidation product of $L A$ [13]) is part of the total scavenging capacity of $L A$ (shown as the crosshatched part). The total scavenging capacity of TNB could not be quantified, because the TNB solution also contained DTNB that interfered with the determination of the total scavenging capacity. Inset: Determination of the observed stoichiometry of the reaction of DTT with $\mathrm{HOCl}$ and the total $\mathrm{HOCl}$ scavenging capacity of DTT. The left y-axis is the remaining concentration of DTT; the right $y$-axis is the remaining concentration of $\mathrm{HOCl}$.

\section{Results}

The stoichiometry of the reaction of $\mathrm{HOCl}$ with several antioxidants appeared to be variable (figure 2). DTT has a stoichiometry of three, TNB has a stoichiometry of 1.3 and $\beta$-LA has a stoichiometry of four. The total scavenging capacity of the tested antioxidants appeared also to be variable (figure 2). The total scavenging capacity of GSH is four and that of LA is seven. All of the tested antioxidants had a total scavenging capacity that was higher than their stoichiometry.

Several antioxidants have been tested in various competition assays (table 1). The activity is expressed as the $\mathrm{EC}_{50^{\prime}}$ the concentration of the antioxidant that reduces the oxidation of the detector by 50 percent. The antioxidant activities DTT and TNB were tested in a competition assay that used MET as detector, using different concentrations of $\mathrm{HOCl}$ (figure 3). At relatively low concentrations of $\mathrm{HOCl}$, TNB had a lower $\mathrm{EC}_{50}$ than DTT, indicating that TNB is a better antioxidant than DTT. However, at high concentrations of $\mathrm{HOCl}$, the $\mathrm{EC}_{50}$ of DTT is lower than that of TNB, which indicates that DTT is a better antioxidant than TNB under this condition. 


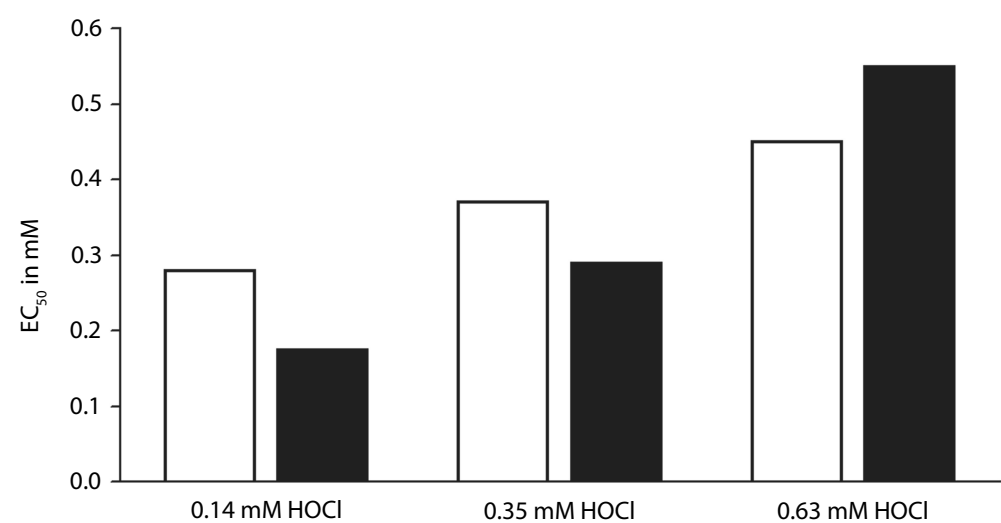

Figure 3. The antioxidant activity of DTT and TNB determined in the MET competition assay for HOCl scavenging. Methionine was used as detector in a concentration of $1 \mathrm{mM}$ and three different initial concentrations of $\mathrm{HOCl}\left([\mathrm{HOCl}]_{0}\right)$ were used. The $E C_{50}$ of DTT (white) increased less than the $E C_{50}$ of TNB (black) at increasing concentration of HOCl.

\section{Discussion}

The activity of antioxidants is usually determined in competition assays. A major problem is that the observed activity of an antioxidant heavily depends on the experimental conditions of the competition assay used. This prompted us to re-examine the fundaments of competition assays. The fundament of a competition assay is competition between antioxidant and detector for the reactive species. In general, it is assumed that the antioxidant activity is primarily governed by the reaction rate constant $\left(\mathrm{k}_{\mathrm{a}}\right)$ of the antioxidant with the reactive species.

Our evaluation indicates that the stoichiometry is involved in the observed antioxidant activity. This can be deduced from the HOCl scavenging activity of DTT and TNB (figure 3). The ranking of these antioxidants appears to depend on the initial concentration of $\mathrm{HOCl}$ in the assay. This can be explained by the relatively high stoichiometry of DTT (3) compared to TNB (1.3). The difference in stoichiometry indicates that in a competition assay relatively more TNB is consumed than DTT at a fixed concentration of HOCl: the decrease in concentration of TNB is higher than that of DTT. This decrease in concentration reduces the observed activity of TNB more than the observed activity of DTT. The reduction in the activity is more pronounced at high initial concentrations of $\mathrm{HOCl}$. The impact of the consumption of the antioxidant on the observed antioxidant activity has been described in detail previously [7]. The faster reacting antioxidant TNB displays a lower antioxidant activity than DTT at a relatively high concentration of $\mathrm{HOCl}$, whereas at a relatively low concentration of $\mathrm{HOCl}$, TNB is the better antioxidant. This shows that the stoichiometry is involved in the observed antioxidant activity and that antioxidant activity is not solely governed by the reaction rate constant of the antioxidant with the reactive species. The impact of the stoichiometry is high at relatively high concentrations of the reactive species. 
The stoichiometry of DTT of three would mean that one molecule of DTT reacts simultaneously with three molecules of $\mathrm{HOCl}$ in a fourth order reaction. Fourth order reactions are however highly unlikely to occur. It is more likely that the observed stoichiometry of DTT is the result of three sequential reactions with a stoichiometry of one. First, a molecule of DTT reacts with one molecule of $\mathrm{HOCl}$ and a product is formed. This first product reacts with a second molecule of $\mathrm{HOCl}$ and a second product is formed. Subsequently, the second product reacts with a third molecule of $\mathrm{HOCl}$. To obtain an observed stoichiometry of three, the reaction rates of the first and the second product with $\mathrm{HOCl}$ have to be higher than that of DTT with $\mathrm{HOCl}$. It has been demonstrated previously that products formed from an antioxidant can be more reactive than the parent antioxidant [8].

When the second product of DTT reacts with the third molecule of $\mathrm{HOCl}$, a third product is formed. From figure 2, it can be deduced that this third product also reacts with $\mathrm{HOCl}$. The observed stoichiometry of three indicates that the third product reacts slower with $\mathrm{HOCl}$ than does DTT. Ultimately, a product is formed that does not react with the reactive species. The number of molecules of reactive species that can be scavenged by one molecule of antioxidant is described as the total scavenging capacity (TSC). The TSC of DTT is 3.8. This means that one molecule of DTT, including all its products, can scavenge on average 3.8 molecules of $\mathrm{HOCl}$. The TSC is the sum of the stoichiometry of the parent antioxidant, of the first product and of all other products formed in subsequent reactions.

GSH appears to have a TSC of four, which is in accordance with previous studies $[9,10]$. This indicates that from the four products that are formed, the first three products also react with $\mathrm{HOCl}$. The TSC of an antioxidant does, however, not correlate with antioxidant activity. For example, LA has a higher total scavenging capacity than GSH (7 and 4, respectively), while in all the assays given in table 1, GSH is the better antioxidant.

The data obtained with GSH show that the products of an antioxidant can also contribute to the observed antioxidant activity. This is illustrated in figure 1. The contribution of the products to the observed antioxidant effect can be estimated by calculating the observed capacity (OCAP ${ }_{50^{\prime}}$ equation 1).

$\operatorname{OCAP}_{50}=1 / 2 \cdot \frac{[\mathrm{HOCl}]_{0}}{E C_{50}}$

Equation 1

The OCAP ${ }_{50}$ describes the average number of molecules of $\mathrm{HOCl}$ that is scavenged by a single molecule of antioxidant (including the products) in a particular assay under $\mathrm{EC}_{50}$ conditions. If $\mathrm{OCAP}_{50}$ is higher than the stoichiometry of the antioxidant, products contribute to the antioxidant activity under $\mathrm{EC}_{50}$ conditions. The $\mathrm{OCAP}_{50}$ has been calculated for several assays (table 2). The OCAP for GSH in the MET assay (0.46) is smaller than the stoichiometry of GSH (which is one). This indicates that there is only competition between the parent antioxidant GSH and the detector MET for HOCl. The first product of GSH reacts too slowly with $\mathrm{HOCl}$, relative to MET, to compete with MET for $\mathrm{HOCl}$. 
Table 2: The observed capacity of GSH, GSSG and LA in several competition assays. The OCAP is calculated using the data given in table 1. The OCAP 50 gives the average number of reactive species (i.e. HOCI) scavenged by the antioxidant at $E C_{50}$ conditions.

\begin{tabular}{llll}
\multicolumn{5}{c}{ OCAP $_{50}$} \\
Detector & GSH & GSSG & LA \\
a -AP & 1.25 & $<0.125$ & 0.83 \\
MET & 0.6 & n.d. & 0.35 \\
LA & 0.5 & n.d. & n.d. \\
DHR123 & 1.9 & 0.27 & 0.5 \\
& n.d. & n.d. & 1.04 \\
AChE & 2.5 & 1.67 & 1.25 \\
Albumin & 2.63 & 0.79 & 1 \\
& 4 & n.d. & 1.16 \\
Luminol & n.d. & n.d. & 2.08
\end{tabular}

n.d.: no data available.

The $\mathrm{OCAP}_{50}$ of GSH in the albumin assay is four. In this assay, four molecules of $\mathrm{HOCl}$ react with one molecule of GSH (and its products). The OCAP 50 equals the TSC of GSH, indicating that albumin reacts too slowly with $\mathrm{HOCl}$ to be able to compete with GSH and the first three products for $\mathrm{HOCl}$. The outcome of this 'competition' assay is the total scavenging capacity of GSH. The difference in extent of the contribution of the products of GSH between the MET assay and the albumin assay depends on the difference between reaction rate of $\mathrm{HOCl}$ with $\mathrm{MET}$ (which is relatively high) relative to that of $\mathrm{HOCl}$ with albumine (which is relatively low) and the intermediate reactivity of the products of GSH. This is schematically outlined in figure 4 .

The evaluation of other competition assays (table 2 ) indicates that in many assays the products of the antioxidant contribute to the antioxidant activity of the parent compound.

In contrast to the prevailing perception, the scavenging activity of an antioxidant is not reflected by the reaction rate of the antioxidant with the reactive species. Although the $k_{a}$ (relative to the reaction rate constant of the detector) determines whether scavenging will occur; the observed antioxidant activity is determined by the number of reactive species scavenged. Therefore, the observed activity of an antioxidant on a molecular level is described more accurately by the OCAP ${ }_{50}$ than the $k_{a}$. The $\mathrm{OCAP}_{50}$ directly gives the actual scavenging activity of an antioxidant molecule (i.e. the average number of radical scavenged by one molecule of antioxidant) and includes the contribution of antioxidant products to the observed activity. The $\mathrm{OCAP}_{50}$ of an antioxidant has a value between zero and its total scavenging capacity. 

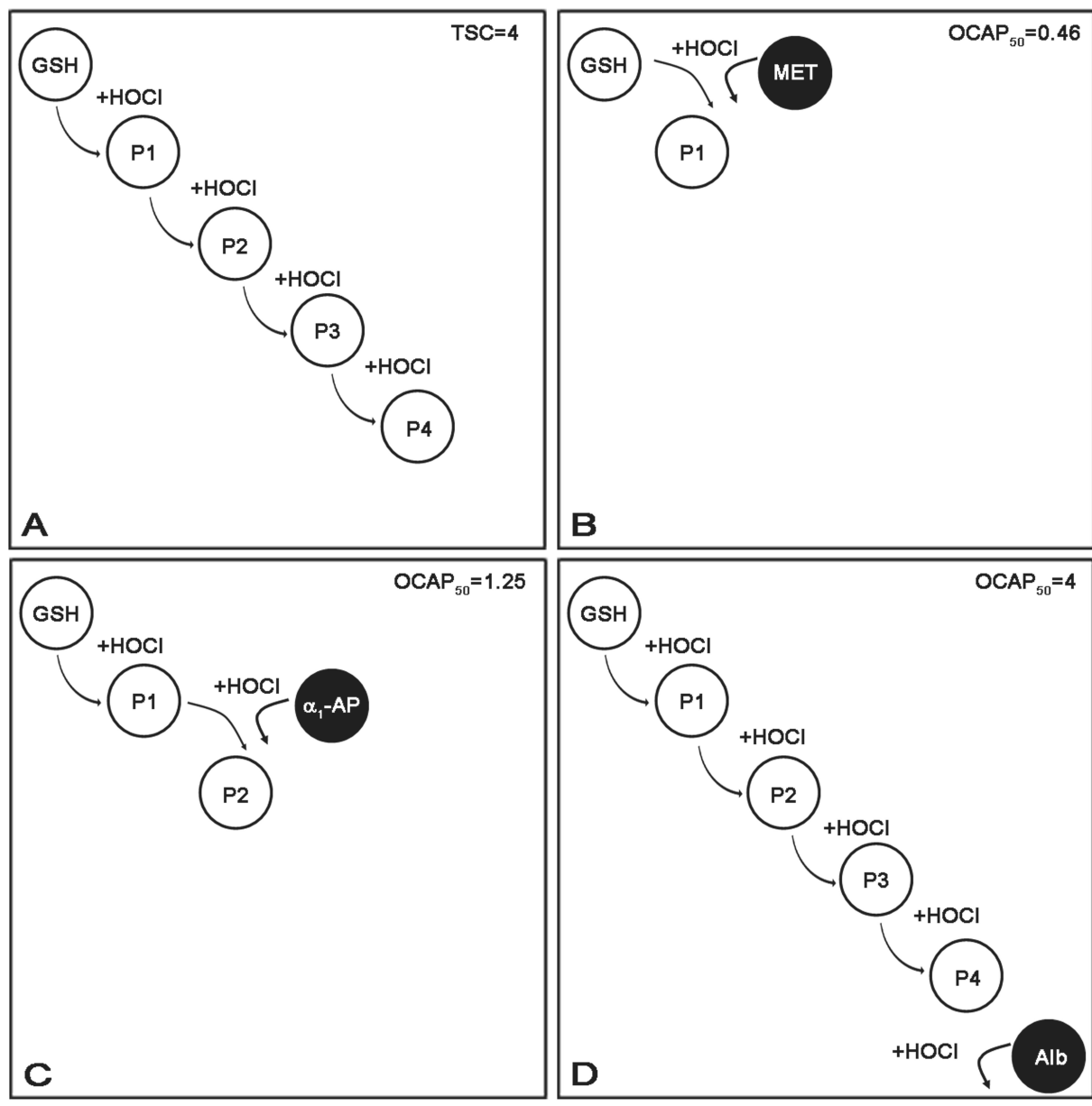

Figure 4. Schematic representation of the $\mathrm{HOCl}$ scavenging by GSH in several assays. A: Total scavenging capacity (TSC) of GSH. The four reaction products (P1 to P4) are shown that are formed in subsequential reactions. It is assumed that the reaction rate constant of subsequent reactions declines. B: GSH in the MET assay. The OCAP of 0.46 indicates that only GSH itself competes with MET for $\mathrm{HOCl}$. The first product (P1) reacts too slowly to compete with MET. C: GSH in the $a_{1}$-AP assay. The OCAP $_{50}$ of 1.25 indicates that GSH reacts much faster than $a_{1}$-AP with $\mathrm{HOCl}$. That the $\mathrm{OCAP}_{50}(1.25)$ is higher than the stoichiometry (1) indicates that also the first product (P1) is able to compete with $a_{1}-A P$ for HOCl. D: GSH in the Albumin (Alb) assay. The antioxidant efficacy of four indicates that GSH and the first three products (P1-P3) react much faster than Alb with $\mathrm{HOCl}$. Only after complete consumption of the third product (P3), Alb will react with the remaining $\mathrm{HOCl}$. The outcome of this 'competition' assay is the TSC of GSH. 
Comparable to the in vitro situation, the products of an antioxidant can also contribute to the activity of antioxidants in vivo. For protection by a compound in vivo, the reaction rate of the reactive species with a compound has to be higher than that of the biotarget. For $\mathrm{HOCl}$, the major biotarget is $\mathrm{a}_{1}$-AP [1]. The results of the competition assay show that the reaction rate of the first product of GSH is sufficiently high to protect $a_{1}-A P\left(O C A P_{50}\right.$ of 1.25$)$, which implies a potential role of this product in vivo.

In vivo, chloramines are detected, which are formed in the reaction of amines with $\mathrm{HOCl}$. Amines do not protect against the inactivation of $\mathrm{a}_{1}-\mathrm{AP}$ by $\mathrm{HOCl}$, because the reaction rate of $\mathrm{HOCl}$ with amines is much lower than that with $a_{1}-A P[11]$. The formation of chloramines in vivo indicates that antioxidants that react faster with $\mathrm{HOCl}$ (e.g. both GSH and the first product of GSH) than amines already have scavenged $\mathrm{HOCl}$. This supports a protective role of products of antioxidants in vivo.

Also in other processes than $\mathrm{HOCl}$ scavenging, products of antioxidants might play a role. For example, the protection provided by the antioxidant quercetin in lipid peroxidation proceeds after all of the quercetin has been consumed [12]. The prolonged protection can only be attributed to the products of quercetin formed.

In conclusion, the activity of a scavenging antioxidant observed in competition assays is not governed by the reaction rate constant of its reaction with the reactive species. The observed stoichiometry and the reactivity of the products of the antioxidant contribute to this observed activity. The observed stoichiometry and the reaction products are included if the antioxidant activity is described by the average number of reactive species scavenged by one molecule of antioxidant, the observed capacity. 


\section{References}

1. Evans, M.D. and Pryor, W.A., Cigarette smoking, emphysema, and damage to alpha 1-proteinase inhibitor. Am. J. Physiol., 1994. 266(6 Pt 1): p. L593-611.

2. Den Hartog, G.J., et al., Efficacy of $\mathrm{HOCl}$ scavenging by sulfur-containing compounds: antioxidant activity of glutathione disulfide? Biol. Chem., 2002. 383(3-4): p. 709-713.

3. Gatto, M.T., et al., Development of a new assay for the screening of hypochlorous acid scavengers based on reversed-phase high-performance liquid chromatography. Biomed. Chromatogr., 2002. 16(6): p. 404-411.

4. Kooy, N.W., et al., Peroxynitrite-mediated oxidation of dihydrorhodamine 123. Free Radic. Biol. Med., 1994. 16(2): p. 149-156.

5. Ching, T.L., de Jong, J., and Bast, A., A method for screening hypochlorous acid scavengers by inhibition of the oxidation of 5-thio-2-nitrobenzoic acid: application to anti-asthmatic drugs. Anal. Biochem., 1994. 218(2): p. 377-381.

6. Haenen, G.R.M.M. and Bast, A., Scavenging of hypochlorous acid by lipoic acid. Biochem. Pharmacol., 1991. 42(11): p. 2244-2246.

7. Balk, J.M., Bast, A., and Haenen, G.R.M.M., Evaluation of the accuracy of antioxidant competition assays: incorrect assumptions with major impact. Free Radic. Biol. Med., 2009. 47(2): p. 135-144.

8. Arts, M.J.T.J., et al., Antioxidant capacity of reaction products limits the applicability of the Trolox Equivalent Antioxidant Capacity (TEAC) assay. Food Chem. Toxicol., 2004. 42(1): p. 45-49.

9. Luo, X., Lu, J.D., and Fu, X.Y., Study of Oxidation of Glutathione Treated with Hypochlorous Acid by Capillary Electrophoresis. Chinese Chemical Letters, 2001. 12(7): p. 623-624.

10. Prütz, W.A., Hypochlorous Acid Interactions with Thiols, Nucleotides, DNA, and Other Biological Substrates. Arch. Biochem. Biophys., 1996. 332(1): p. 110-120.

11. Peskin, A.V. and Winterbourn, C.C., Kinetics of the reactions of hypochlorous acid and amino acid chloramines with thiols, methionine, and ascorbate. Free Radic. Biol. Med., 2001. 30(5): p. 572-579.

12. Haenen, G.R.M.M., et al., Structure and activity in assessing antioxidant activity in vitro and in vivo: A critical appraisal illustrated with the flavonoids. Environ. Toxicol. Pharmacol., 2006. 21(2): p. 191-198.

13. Biewenga, G.P., Haenen, G.R.M.M., and Bast, A., The pharmacology of the antioxidant lipoic acid. Gen. Pharmacol., 1997. 29(3): p. 315-331.

14. Rezk, B.M., et al., Lipoic acid protects efficiently only against a specific form of peroxynitrite-induced damage. J. Biol. Chem., 2004. 279(11): p. 9693-9697.

15. Gomes, A., et al., 2-Styrylchromones: novel strong scavengers of reactive oxygen and nitrogen species. Bioorg. Med. Chem., 2007. 15(18): p. 6027-6036.

16. Yan, L.J., et al., Efficacy of hypochlorous acid scavengers in the prevention of protein carbonyl formation. Arch. Biochem. Biophys., 1996. 327(2): p. 330-334.

17. Gomes, A., et al., Antioxidant activity of beta-blockers: an effect mediated by scavenging reactive oxygen and nitrogen species? Bioorg. Med. Chem., 2006. 14(13): p. 4568-4577.

18. Costa, D., et al., Inhibition of human neutrophil oxidative burst by pyrazolone derivatives. Free Radic. Biol. Med., 2006. 40(4): p. 632-640. 

$d x_{1}=-k_{j} \cdot x_{1}$
$d x_{2}=k_{2} w_{1}$ 


\section{Chapter 5}

\section{Increased citrate levels in non-alcoholic steatohepatitis:}

effect on CYP2E1 


\section{Abstract}

The prevalence of non-alcoholic fatty liver disease and non-alcoholic steatohepatitis (NASH) is high in the western countries and an effective therapy does not exist. The development op non-alcoholic steatohepatitis consists of two hits; fatty acid accumulation and the occurrence of inflammation. The effect of fatty acid exposure to hepatocytes (HepG2) on the citrate levels was evaluated by liquid chromatography-tandem mass spectrometry (LC/MS-MS). The citrate concentrations in blood plasma of patients with proven NASH were determined by LC/MS-MS. The effect of citrate on the production of superoxide by cytochrome P450 2E1 (CYP2E1) was determined by electron spin resonance and reduction of ferricytochrome $\mathrm{C}$.

Citrate levels were elevated in patients with NASH. The citrate levels increase on exposure of HepG2 cells to fatty acids. CYP2E1 is responsible for the production of superoxide and this production can partially be inhibited by superoxide dismutase. Citrate did not enhance the production of superoxide by CYP2E1.

Citrate levels are elevated upon exposure to fatty acids. It did not enhance the production of superoxide by CYP2E1. Citrate probably contributes indirectly to the development of NASH. High plasma levels of citrate in NASH could facilitate the diagnosis of NASH. 


\section{Introduction}

Non-alcoholic fatty liver disease (NAFLD) is featured as a hepatic manifestation of metabolic syndrome [1]. In developed countries the prevalence of NAFLD is $20-30 \%$ in the general population [2]. Its manifestation ranges from steatosis to non-alcoholic steatohepatitis (NASH) and cirrhosis. General measures to prevent the development of NASH from NAFLD that have been suggested are weight reduction in obese patients, to increase physical activity and to avoid the use of alcohol. Pathologies that occur concomitantly with NAFLD or NASH, like diabetes, high blood pressure or high cholesterol levels, can be treated efficiently. However specific therapies for NAFLD or NASH do not exist.

Involvement of reactive oxygen species (ROS) in the etiology of NASH is apparent, although the origin of these ROS is still unclear. It is known for more than a decade that cytochrome P450 2E1 (CYP2E1) is increased in patients with NASH [3] and may contribute to the pathogenesis of NASH [4]. Recently, certain allele variants in CYP2E1 have been associated with liver injury in NASH [5]. The iso-enzyme CYP2E1 shows a strong oxidase activity in which the cytochrome P450 reaction cycle uncouples and oxygen is partially reduced to form the superoxide anion radical $\left(\mathrm{O}_{2}^{-}\right)$. Compounds that interact with cytochrome P450 without being oxidized might stimulate this uncoupling or oxidase activity of cytochrome P450 [6].

It has been reported recently that citrin deficiency leads to steatogenesis and mimics NAFLD [7]. This was explained by a compensatory up-regulation of the malate-citrate shuttle, which increases citrate levels in the cytosol. Citrate is subsequently converted into acetyl-CoA by ATP-citrate lyase and leads to overproduction of fatty acids in hepatocytes.

A high load of fatty acids, which is present in patients with NASH, might lead to citrate accumulation in the mitochondria, which subsequently could result in high cytosolic citrate levels. Therefore, we hypothesized that citrate levels may be high in NAFLD and NASH patients.

The oxidase activity of haem proteins could be triggered by a compound that is difficult to oxidize [8]. The highly oxidized structure of citrate suggests that it will be difficult to oxidize by CYP2E1. We therefore investigated if citrate could be the trigger in the oxidase activity of CYP2E1, thus playing a key role in the development of NASH.

\section{Methods}

Materials

All chemicals used were of analytical grade. The spin trap 5-diethoxyphosphoryl-5-methyl-1pyrroline N-oxide (DEPMPO) was purchased from Calbiochem (Darmstadt, Germany). Acetylated ferricytochrome C, Cu/Zn-superoxide dismutase (SOD), palmitic acid and bovine serum albumin (BSA) are obtained from Sigma (St. Louis, USA).

Patients and tissue sampling

In the samples of patients presenting with liver enzyme elevations, viral serology and extended metabolic and immunologic blood and urine investigations were performed including a liver biopsy. 
Patients, who did meet the criteria for NAFLD/NASH and gave informed consent to use their data in this study, were enrolled in this study. The data were collected according to the routine clinical practice of our outpatient clinic. None of the participating patients had an active viral disease, alcohol liver disease, haemochromatosis, copper storage disease, alpha-1 antitrypsine deficiency or drug induced liver disease. None of the patients had liver cirrhosis.

Measurement of citrate levels in fatty acid loaded cells

The human hepatoma cell line, HepG2, was obtained from American Type Culture Collection (United Kingdom). The cells were cultured in minimum essential medium containing 10\% fetal bovine serum (Invitrogen, Breda, The Netherlands) according to Chen et al. [9]. HepG2 cells were exposed for 24 hours to palmitic acid ( $8 \mathrm{mM}$ ) dissolved in serum free medium containing 10\% bovine serum albumin as described by Joshi-Barve et al. [10]. After incubation, citrate levels in the medium were determined using LC-tandemMS, adapted from Keevil et al. [11].

The cells were fixed with 10\% formalin and washed with 60\% isopropanol. Fat accumulation in HepG2 cells was shown by staining with Oil Red O. After subsequent rinsing of the culture dishes with distilled water, a counterstaining with hematoxylin was applied and visualized via light microscopy. Animal pretreatment and preparation of liver microsomes

In Lewis rats, the level of CYP2E1 was upregulated by providing the rats with acetone in the drinking water $(1 \%(v / v))$ for seven days and depriving them from food 24 hours before sacrifice $[12,13]$. The rats were anaesthetized with $\mathrm{CO}_{2} / \mathrm{O}_{2}$ (ratio 2:3) and decapitated. The protocol was approved by the institutional ethical review board on experiments with animals.

The livers were removed and homogenized in ice-cold potassium phosphate buffer $(50 \mathrm{mM}$, containing $22.8 \mathrm{mM}$ potassium chloride and $3.4 \mathrm{mM}$ EDTA, pH 7.4) and centrifuged at $10000 \times \mathrm{g}$ for $20 \mathrm{~min}$ at $4^{\circ} \mathrm{C}$. The supernatant was centrifuged at $10000 \mathrm{xg}$ for $20 \mathrm{~min}$ at $4^{\circ} \mathrm{C}$. The microsomes were washed and suspended to a concentration of 2 grams liver per milliliter buffer and stored at $-80^{\circ} \mathrm{C}$ until use.

Measurement of $\mathrm{O}_{2}^{--}$formation via the acetylated ferricytochrome $\mathrm{C}$ assay

The formation microsomal $\mathrm{O}_{2}^{-}$was measured by the reduction of acetylated ferricytochrome $\mathrm{C}$. Acetylated, instead of native, ferricytochrome $\mathrm{C}$ was used in order to prevent enzymatic reduction by P450-reductase [14]. Microsomes (from $0.0625 \mathrm{gr}$ liver) in $50 \mathrm{mM}$ potassium phosphate buffer ( $\mathrm{pH}$ 7.4) were incubated at $37^{\circ} \mathrm{C}$ with $\mathrm{MgCl}_{2}(3.3 \mathrm{mM})$, glucose-6-phosphate (3.3 mM), NADP+ (1.3 mM), glucose-6-phosphate dehydrogenase $(0.4 \mathrm{U} / \mathrm{mL})$ and $100 \mu \mathrm{M}$ acetylated ferricytochrome $\mathrm{C}$. Ferrous cytochrome $\mathrm{C}$ is measured spectrophotometrically at $550 \mathrm{~nm}$. To confirm the involvement of $\mathrm{O}_{2}^{-}$in the reduction of acetylated ferricytochrome $\mathrm{C}, \mathrm{Cu} / \mathrm{Zn}-\mathrm{SOD}(2 \mathrm{U} / \mathrm{mL})$ was used.

Measurement of $\mathrm{O}_{2}^{-}$- formation with ESR

Similar conditions as in the ferricytochrome $\mathrm{C}$ assay were used to measure the formation of $\mathrm{O}_{2}^{-}$via ESR on a Brucker EMX spectrometer. A concentration of $50 \mathrm{mM}$ DEPMPO as a specific $\mathrm{O}_{2}^{-}$- spin trap was used [15]. A center field of $3490 \mathrm{G}$, sweep width of $140 \mathrm{G}$ and 10 scans were used. 
Table 1: Characteristics of patients diagnosed with NASH.

\begin{tabular}{|c|c|c|c|c|c|c|}
\hline Variables & Patient 1 & Patient 2 & Patient 3 & Patient 4 & Patient 5 & Patient 6 \\
\hline Age (yr) & 58 & 57 & 70 & 65 & 44 & 44 \\
\hline Gender (m/f) & $\mathrm{m}$ & $\mathrm{m}$ & $f$ & $\mathrm{~m}$ & $f$ & $f$ \\
\hline Body mass index $\left(\mathrm{kg} / \mathrm{m}^{2}\right)$ & 28.0 & 25.4 & 26.8 & 26.8 & 30 & 38 \\
\hline Alcohol intake/ day & 0 & 2 & 0 & 0 & 0 & 0 \\
\hline Systolic blood pressure & 142 & 140 & 160 & 120 & 145 & 120 \\
\hline Diastolic blood pressure & 84 & 90 & 80 & 90 & 85 & 84 \\
\hline $\begin{array}{l}\text { Gamma-GT } \\
(-50 \mathrm{U} / \mathrm{L})\end{array}$ & 139 & 341 & 49 & 461 & 189 & 92 \\
\hline $\begin{array}{l}\text { AF } \\
(0-140 \mathrm{U} / \mathrm{L})\end{array}$ & 84 & 119 & 121 & 145 & 140 & 91 \\
\hline $\begin{array}{l}\text { ASAT } \\
(0-35 \mathrm{U} / \mathrm{L})\end{array}$ & 76 & 84 & 82 & 52 & 39 & 42 \\
\hline $\begin{array}{l}\text { ALAT } \\
(0-45 \mathrm{U} / \mathrm{L})\end{array}$ & 121 & 84 & 172 & 77 & 86 & 52 \\
\hline Total bilirubin $(0-20 \mu \mathrm{g} / \mathrm{L})$ & 12 & 32 & 9.6 & 28 & 22 & 17.4 \\
\hline PT (1.0 INR) & 1.0 & 1.1 & 1.1 & 1.2 & 1.0 & 1.0 \\
\hline $\begin{array}{l}\text { AT III } \\
(80-120 \%)\end{array}$ & 103 & 81 & 98 & 63 & 96 & 78 \\
\hline $\begin{array}{l}\text { Total cholesterol } \\
(5-6.4 \mathrm{mmol} / \mathrm{l})\end{array}$ & 7.0 & 6.0 & 6.1 & 7.1 & 4.3 & 4.9 \\
\hline HDL cholesterol (1-2 mmol/l) & 1.3 & 1.4 & 1.2 & 1.6 & 1.2 & 1.6 \\
\hline LDL cholesterol (3.5-4.4 mmol/I) & 4.4 & 3.9 & 4.1 & 4.2 & 2.6 & 2.8 \\
\hline Triglyceride & 2.90 & 1.64 & 1.82 & 2.93 & 1.00 & 1.21 \\
\hline Fasting glucose & 5.7 & 7.1 & 7.3 & 9.7 & 5.2 & 6.7 \\
\hline $\begin{array}{l}\text { Albumine } \\
(32-47 \mathrm{~g} / \mathrm{l})\end{array}$ & 44.4 & 42.0 & 43.2 & 40.0 & 44.5 & 38.6 \\
\hline Uric acid $(0.20-0.42 \mu \mathrm{mol} / \mathrm{l})$ & - & 0.29 & 0.38 & 0.26 & 0.37 & 0.35 \\
\hline $\begin{array}{l}\text { Citrate plasma } \\
(52-106 \mu \mathrm{mol} / \mathrm{l})\end{array}$ & 138 & 146 & 201 & 210 & 178 & 176 \\
\hline
\end{tabular}

Abbreviations: ALAT: alanine aminotransaminase, ASAT: aspartate aminotransaminase, gamma-GT: gammaglutamyl transferase, AF: alkaline phosphatase, AT III: antithrombine three; PT, prothrombine time;

\section{Results}

Patients

In order to investigate whether citrate plasma levels were increased in NASH, 6 patients were selected for this study, (3 males and 3 female) with a mean age of 56 years (range 44-70 years). The patients did not take antihypertensive, lipid and glucose lowering medication at time of diagnosis. None of the patients had used drugs which could induce liver steatosis. Patients' characteristics are summarized in table 1. Most of the patients had one or more characteristics of the metabolic syndrome according to the criteria of the American Heart Society; five patients had an elevated fasting glucose level $(1-4,6)$, three patients had elevated blood pressure $(2,4,5)$, three patients showed elevated triglyceride levels $(1,3,4)$.

All patients had overweight; two patients were obese, with mainly abdominal fat deposition. 
Combined liver enzyme abnormalities were found in all patients without signs of cholestasis. The abdominal sonography showed bright liver in all patients and the liver biopsy taken, classified according to the NAS score [16], confirming NASH in four out of six patients (table 2).

Table 2: Liver biopsy according to NAS scoring system [15]

\begin{tabular}{|c|c|c|c|c|c|c|}
\hline NAS score & Patient 1 & Patient 2 & Patient 3 & Patient 4 & Patient 5 & Patient 6 \\
\hline \multicolumn{7}{|l|}{ steatosis } \\
\hline grade & 1 & 3 & 2 & 2 & 2 & 2 \\
\hline location & 1 & 3 & 3 & 1 & 1 & 1 \\
\hline microvesicular & 1 & 1 & 1 & 1 & 1 & 1 \\
\hline \multicolumn{7}{|l|}{ fibrosis } \\
\hline stage & 0 & 3 & 1 & 1 & 0 & 1 \\
\hline \multicolumn{7}{|l|}{ inflammation } \\
\hline lobular inflammation & 1 & 1 & 1 & 1 & 1 & 2 \\
\hline microgranulomas & 0 & 1 & 0 & 0 & 0 & 1 \\
\hline large lipogranulomas & 0 & 0 & 0 & 1 & 1 & 0 \\
\hline portal inflammation & 0 & 0 & 0 & 0 & 0 & 1 \\
\hline ballooning & 0 & 1 & 0 & 0 & 0 & 1 \\
\hline acidophil bodies & 0 & 0 & 0 & 0 & 0 & 0 \\
\hline pigmented macrophages & 0 & 1 & 1 & 1 & 1 & 1 \\
\hline megamitochondria & 0 & 0 & 0 & 0 & 0 & 0 \\
\hline mallory hyaline & 0 & 0 & 0 & 0 & 0 & 0 \\
\hline glycogenated nuclei & 0 & 0 & 0 & 0 & 0 & 1 \\
\hline NAS score & 2 & 5 & 5 & 5 & 3 & 5 \\
\hline
\end{tabular}

Citrate formation in fatty acid loaded HepG2 cells

Exposure of human hepatoma (HepG2) cells to $8 \mathrm{mM}$ palmitic acid for 24 hours resulted in fatty acid accumulation in the cells and in extensive citrate levels in the culture medium (figure 1).
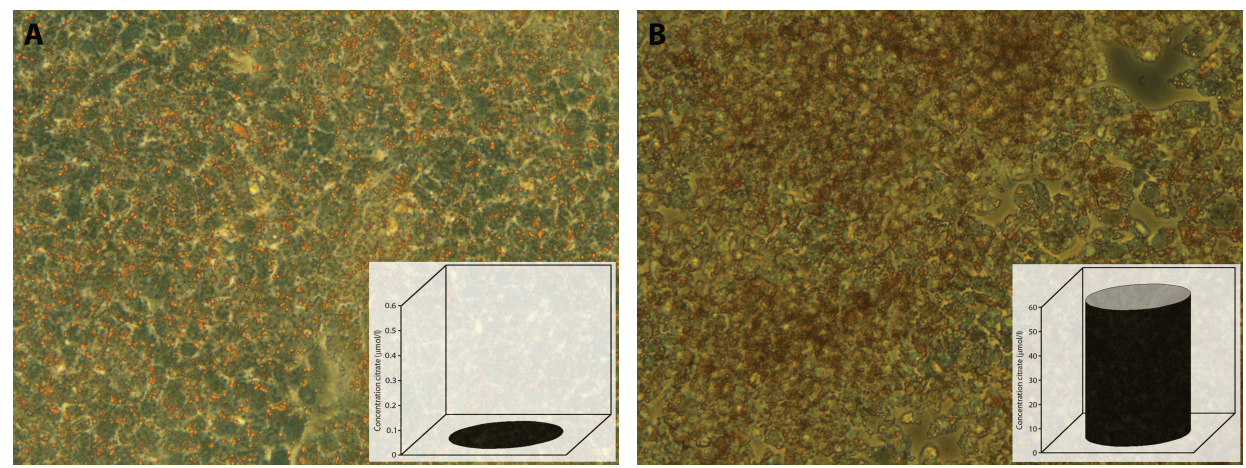

Figure 1. A 24 hr incubation of Hep G2 cells with $8 \mathrm{mM}$ palmitic acid leads to intracellular lipid accumulation as shown by staining with Oil Red O. Cells (A, control; B, incubated with palmitic acid) were photographed under light microscopy at a magnification of 20 times. The inset shows the citrate level in the medium after incubation. 
Formation of $\mathrm{O}_{2}^{-}$- in rat liver microsomes

Liver microsomes were prepared from rats in which CYP2E1 was induced. With an NADPH regenerating system, electrons were provided to the microsomal enzyme system. CYP2E1 is known to possess oxidase activity upon stimulation with $\mathrm{NADPH}$. In this activity, one electron reduced oxygen uncouples from the enzyme $[6,14]$. This is measured via the reduction of acetylated ferricytochrome $C$ at $550 \mathrm{~nm}$. The acetylated form is used in order to prevent possible enzymatic reduction.

The reduction of acetylated ferricytochrome $C$ is inhibited, although not fully, by SOD indicating that $\mathrm{O}_{2}{ }^{-}$is indeed involved (figure 2). The initial rate of the formation of $\mathrm{O}_{2}{ }^{-}$in the presence of $10 \mathrm{mM}$ citrate is indicated in the inset in figure 2. Citrate, oxaloacetate, pyruvate and malate, concentration of $10 \mathrm{mM}$, did not stimulate the SOD inhibitable reduction of ferricytochrome C (data not shown). The formation of $\mathrm{O}_{2}^{-}$- in liver microsomes was confirmed using the specific $\mathrm{O}_{2}^{--}$spintrap DEPMPO (figure 3). The formation of the DEPMPO- $\mathrm{O}_{2}^{-}$- ESR signal in rat liver microsomes with an NADPH regenerating system could be fully prevented by adding SOD.

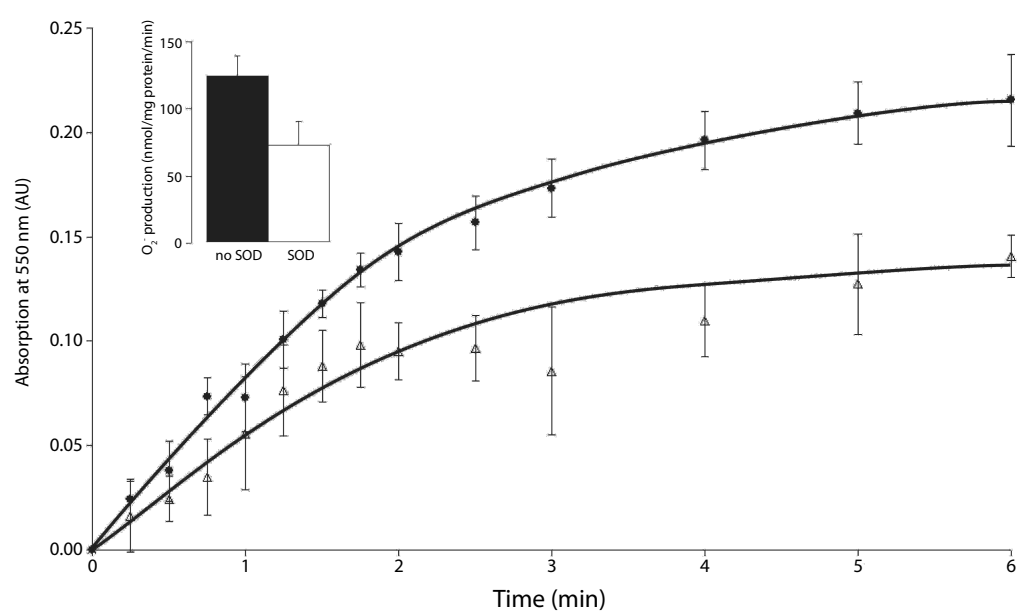

Figure 2. The reduction of acetylated ferricytochrome $C$ in the presence of $10 \mathrm{mM}$ citrate in rat liver microsomes with induced CYP2E1 as measured at $550 \mathrm{~nm}$. SOD partly inhibits the reduction of ferricytochrome $C$. The inset shows the calculated rate of initial formation of $\mathrm{O}_{2}^{--}$in the presence and absence of SOD using a molar extinction coefficient of 21 $\mathrm{mM}^{-1} . \mathrm{cm}^{-1}$ for ferrouscytochrome $\mathrm{C}$. 


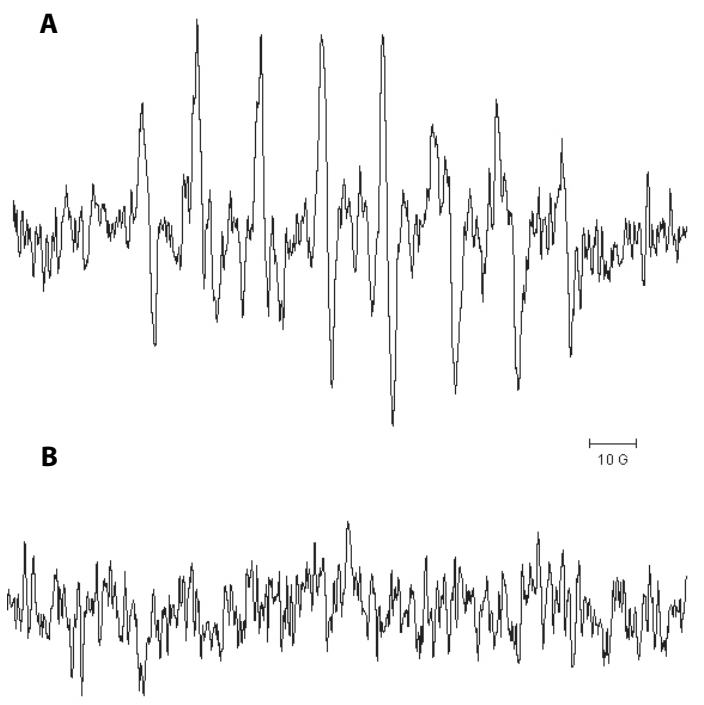

Figure 3. The characteristic ESR spectrum of DEPMPO with $\mathrm{O}_{2}^{--}$in rat liver microsomes (A) and the effect of SOD on the stimulated DEPMPO signal (B).

\section{Discussion}

The pathogenesis of NAFLD and NASH is generally explicated by the so-called 'two hit theory'. The first hit is formed by steatosis, the accumulation of fatty acids in the liver. The second hit is more diffuse and less well characterized and includes the peroxidation of these fatty acids. The peroxidation leads to oxidative stress, which results in the induction of pro-inflammatory molecules and pro-apoptotic molecules such as Fas ligand. Since CYP2E1 is upregulated in NASH, it seems to play a pivotal role in the pathogenesis of NASH [17].

The 'two hit theory' thus provides a general framework to understand NASH. However, the primary successive triggers that associate steatosis to NASH are not fully defined yet. Clearly, NASH begins with an imbalance between energy intake by the diet and consumption by physical exercise. High fuel intake (fat, carbohydrates or proteins) leads to acetyl-CoA as a common metabolic intermediate. In the mitochondria, acetyl-CoA is oxidized in the citric acid cycle [18]. The first compound formed in the citric acid cycle is citrate. It is formed by the reaction of acetyl-CoA with oxaloacetate. This reaction is catalyzed by citrate synthase and has a $\mathrm{G}^{0^{\prime}}$ of $-31.4 \mathrm{~kJ} \mathrm{~mol}{ }^{-1}$. The citrate is subsequently isomerised to isocitrate via cis-aconitate. The dehydration reaction of citrate to cis-aconitate is catalyzed by aconitase which has a $\mathrm{G}^{\circ}$ of $+8.4 \mathrm{~kJ} \mathrm{~mol}^{-1}[19]$. Fuelling the citric acid cycle may thus lead to an accumulation of citrate. Several arguments have indeed been provided to typify NASH as a mitochondrial disease. Interestingly, a recent study demonstrated that citrin deficiency mimics NAFLD [7]. Deficiency of the aspartate-glutamate carrier, citrin, causes loss of the malate-aspartate shuttle and a compensatory up-regulation of the malate-citrate shuttle, resulting in increased citrate levels in the cytosol of the hepatocyte [7]. 
Incubation of HepG2 cells with 8 mM palmitic for 24 hrs lead to intracellular lipid accumulation as demonstrated by staining with Oil Red $O$. This treatment also resulted in an increase in citrate levels in the medium (figure 1). Apparently, increased citrate levels are associated with NASH.

In NASH patients, the plasma citrate levels are strongly increased and range from 138-210 $\mu \mathrm{M}$ compared to reference levels of 52-106 $\mu \mathrm{M}$. Komatsu et al. [7] suggested that in the case of citrin deficiency, the large amounts of citrate that arise may simply be converted to acetyl-CoA by ATPcitrate lyase and via this metabolic pathway lead to overproduction of fatty acids in hepatocytes. Due to the high load of fatty acids, citrate could accumulate inside the mitochondria. These increased levels of citrate could be responsible for increased transport of citrate to the cytosol, providing ATPcitrate lyase with the substrates necessary for lipogenesis which finally exacerbate the steatosis.

There is ample evidence for oxidative stress in NAFLD or NASH, both in experimental models as well as in patients. High levels of fatty acids in the fatty liver fuel the process of lipid peroxidation, which damage hepatic cellular membranes and perpetuate oxidative stress. The precise sparking mechanism that initiates the inflammation process that turns the benign NAFLD into NASH, has however not been characterized.

It is known that certain iso-enzymes of hepatic cytochrome P450, viz. CYP2E1 and CYP4A are induced in NASH, both are involved in oxidative metabolism of fatty acids. Cytochrome P450 activity as a whole include mono-oxygenase, dioxygenase and oxidase activity [6]. Particularly CYP2E1, which is induced in both NAFLD and NASH [20] expresses intense oxidase action [21]. In this oxidase activity, the activated oxygen molecule in the haem protein cytochrome P450 is not incorporated in the oxidation of a substrate, but rather uncouples from the reaction cycle as the reduced form $\mathrm{O}_{2}^{-}$[13]. Previously, it has been suggested that this uncoupling reaction might form a final way to catalyze oxidation of xenobiotics that cannot be metabolized via the mono-oxygenase pathway through one of the cytochrome P450 iso-enzymes [6]. This is feasible because $\mathrm{O}_{2}^{-}$both enzymatically (via superoxide dismutase) or non-enzymatically dismutates to $\mathrm{H}_{2} \mathrm{O}_{2}$, which may generate the reactive oxidizing hydroxyl radical. This hydroxyl radical is able to oxidize xenobiotics.

It was demonstrated using the reduction of acetylated ferricytochrome P450 (figure 2) and the $\mathrm{O}_{2}$. specific spin trap DEPMPO (figure 3) that microsomal CYP2E1 produced superoxide. Both signals could be inhibited by co-incubated SOD. Citrate did not increase the oxidase activity of microsomal CYP2E1, despite its high levels and the difficulty to further oxidize the molecule.

High levels of citrate, which are associated with NASH, may fuel the continuous synthesis of fatty acids and exacerbate the steatosis. Whether citrate is involved in the mechanism that triggers the cellular production of ROS and thus influences cellular viability is uncertain.

The present finding that citrate levels are increased in NAFLD and NASH patients could facilitate the diagnosis of NASH and could provide detailed information of liver function in those patients. 


\section{Acknowledgements}

We thank Ann Driessen and Celien Vreuls from the Department of Pathology of the Maastricht University Medical Centre, who contributed to the patients' pathology data, and Marjon Ortmans for citrate measurements. 


\section{References}

1. Marchesini, G., et al., Nonalcoholic fatty liver disease: a feature of the metabolic syndrome. Diabetes, 2001. 50(8): p. 1844-1850.

2. Jimba, S., et al., Prevalence of non-alcoholic fatty liver disease and its association with impaired glucose metabolism in Japanese adults. Diabet. Med., 2005. 22(9): p. 1141-1145.

3. Weltman, M.D., et al., Hepatic cytochrome P450 $2 \mathrm{E} 1$ is increased in patients with nonalcoholic steatohepatitis. Hepatology, 1998. 27(1): p. 128-133.

4. Robertson, G., Leclercq, I., and Farrell, G.C., Nonalcoholic steatosis and steatohepatitis. II. Cytochrome P-450 enzymes and oxidative stress. Am J Physiol Gastrointest Liver Physiol, 2001. 281(5): p. G1135-1139.

5. Varela, N.M., et al., Study of cytochrome P450 2E1 and its allele variants in liver injury of nondiabetic, nonalcoholic steatohepatitis obese women. Biol. Res., 2008. 41(1): p. 81-92.

6. Bast, A., Is formation of reactive oxygen by cytochrome P-450 perilous and predictable? Trends Pharmacol. Sci., 1986. 7: p. 266-270.

7. Komatsu, M., et al., Citrin deficiency as a cause of chronic liver disorder mimicking non-alcoholic fatty liver disease. J. Hepatol., 2008. 49(5): p. 810-820.

8. Gorren, A.C. and Mayer, B., Nitric-oxide synthase: a cytochrome P450 family foster child. Biochim. Biophys. Acta, 2007. 1770(3): p. 432-445.

9. Chen, J., et al., In vitro evaluation of growth and anabolism for C3A/HepG2 hepatoma cells with logistic equation and linear regression expression. Transplant. Proc., 2001. 33(1-2): p. 656-657.

10. Joshi-Barve, S., et al., Palmitic acid induces production of proinflammatory cytokine interleukin- 8 from hepatocytes. Hepatology, 2007. 46(3): p. 823-830.

11. Keevil, B.G., et al., Measurement of citrate in urine using liquid chromatography tandem mass spectrometry: comparison with an enzymatic method. Ann. Clin. Biochem., 2005. 42(Pt 5): p. 357-363.

12. Koop, D.R., Chernosky, A., and Brass, E.P., Identification and induction of cytochrome P450 $2 E 1$ in rat Kupffer cells. J. Pharmacol. Exp. Ther., 1991. 258(3): p. 1072-1076.

13. Ronis, M.J., et al., Expression and distribution of cytochrome P450 enzymes in male rat kidney: effects of ethanol, acetone and dietary conditions. Biochem. Pharmacol., 1998. 55(2): p. 123-129.

14. Kuthan, H. and Ullrich, V., Oxidase and oxygenase function of the microsomal cytochrome P450 monooxygenase system. Eur. J. Biochem., 1982. 126(3): p. 583-588.

15. Dikalov, S.I., et al., Production of extracellular superoxide by human lymphoblast cell lines: comparison of electron spin resonance techniques and cytochrome C reduction assay. Biochem. Pharmacol., 2007. 73(7): p. 972-980

16. Kleiner, D.E., et al., Design and validation of a histological scoring system for nonalcoholic fatty liver disease. Hepatology, 2005. 41(6): p. 1313-1321.

17. Lieber, C.S., CYP2E1: from ASH to NASH. Hepatol Res, 2004. 28(1): p. 1-11.

18. Pessayre, D. and Fromenty, B., NASH: a mitochondrial disease. J. Hepatol., 2005. 42(6): p. 928-940.

19. Berg, J.M., Tymoczko, J.L., and Stryer, L., Biochemistry. Sixth Edition ed. 2006, New York: W.H. Freeman and Company.

20. Chtioui, H., et al., Expression and activity of the cytochrome P450 $2 \mathrm{E} 1$ in patients with nonalcoholic steatosis and steatohepatitis. Liver Int, 2007. 27(6): p. 764-771.

21. Caro, A.A. and Cederbaum, A.I., Oxidative stress, toxicology, and pharmacology of CYP2E1. Annu. Rev. Pharmacol. Toxicol., 2004. 44: p. 27-42. 
$d x_{1}=-k_{j} \cdot x_{1}$
$d x_{2}=k_{2} w_{1}$ 


\section{Chapter 6}

\section{Substrate inhibition of CYP2E1 activity is primarily determined}

by lipophilicity 


\section{Abstract}

Non-alcoholic steatohepatitis (NASH) is accompanied with significant upregulation of the cytochrome P450 2E1 (CYP2E1). CYP2E1 is capable of producing reactive oxygen species, which trigger the inflammation response and formation of fibrosis. To prevent the exacerbation of this liver damage, the production of reactive oxygen species can be decreased by inhibition of CYP2E1. In the search for compounds that could inhibit CYP2E1 activity, the functional requirements for inhibition were identified. Therefore, a series of primary alcohols, secondary alcohols, ketones, aldehydes and carboxylic acids up to a chain length of eight carbon atoms were tested for their inhibitory potential. The carboxylic acids (small fatty acids) showed the weakest inhibition of CYP2E1 activity, which correlates to the relatively low lipophilicity of those compounds compared to those with other functional groups. Within the other series of compounds tested, a strong correlation between the lipophilicity and the inhibition of CYP2E1 activity was found. It is concluded that lipophilicity is a major determinant for CYP2E1 inhibition. 


\section{Introduction}

In Western countries, obesity will reach epidemic proportions. Obesity is a major risk factor for many diseases, including non-alcoholic fatty liver disease (NAFLD). In 2002, the prevalence of NAFLD in the general population was estimated at 10-24 percent and 57-74 percent in obese persons [1]. NALFD can progress to non-alcoholic steatohepatitis (NASH) and the prevalence of NASH is estimated at 2-3 percent of the general population in the western countries [2].

The livers of patients diagnosed with NAFLD and NASH show massive accumulation of fatty acids in the hepatocytes. This steatosis is the first'hit'in the development of NASH [3]. Normally, fatty acids are primarily stored in the adipose tissue and only minor amounts are stored in the hepatocytes. In the hepatocytes, fatty acids are metabolized via $\beta$-oxidation in the mitochondria. In the livers of NASH patients, abnormalities of the mitochondria are seen (like mega mitochondria [4]), which indicate that the mitochondria cannot cope with the amount of fatty acids present in the hepatocytes. This impairment of function of the mitochondria results in oxidative stress, which is believed to be the second 'hit' in the development of NASH [3].

In NASH, additional pathways of fatty acid metabolism are upregulated; i.e. the peroxisomal pathway (via peroxisome proliferator-activated receptors [5]) and the $\omega$-hydroxylation pathway. A pivotal enzyme in the $\omega$-hydroxylation pathway is cytochrome P450 2E1 (CYP2E1), primarily present in the endoplasmatic reticulum [6-8]. This enzyme in particular is upregulated in patients with NASH [9-11]. CYP2E1 oxidizes its substrates (denoted as RH), which requires oxygen and electrons donated by $\mathrm{NADPH}$ to form the oxidized substrate $(\mathrm{ROH})$. As an intermediate in the monooxygenase activity of CYP2E1, a high-energetic $\mathrm{Fe}^{2+}-\mathrm{O}_{2} \cdot \mathrm{RH}$ complex is formed. This high-energetic complex is relatively unstable and easily dissociates, which yields superoxide radicals [10]. CYP2E1 can also produce reactive oxygen species (ROS), when it is reduced without the binding of a substrate to its catalytic site, which is known as 'futile cycling' [12]. The formation of ROS by failing oxidation or futile cycling could trigger the second 'hit' in NASH.

Based on the etiology of the disease the second hit in NASH, the formation of ROS, could be prevented by inhibition of CYP2E1 activity. The objective of the present study is to identify the major functional requirements of CYP2E1 inhibition. Therefore, a series of compounds with various functional groups was tested for their ability to inhibit CYP2E1.

\section{Methods}

Chemicals

4-nitrophenol (4-NP), 4-nitrocatechol (4-NC), mono- and dipotassiumphosphate, D-glucose-6phosphate, glucose-6-phosphate dehydrogenase (type XI), $\beta$-nicotinamide adenine dinucleotide phosphate ( $\beta$-NADP), primary alcohols, secondary alcohols, 2-ketones, aldehydes and carboxylic acids (all up to a chain length of eight carbon atoms) were obtained from Sigma-Aldrich (St. Louis, USA). Magnesium chloride was purchased from Merck (Darmstadt, Germany). All other chemicals used were of analytical grade. 
Animals

The expression of CYP2E1 in the livers of Lewis rats was upregulated using a combination of the treatment of Koop et al. [13] and the treatment described by Ronis et al. [14]. The rats received acetone containing drinking water $(1 \%(\mathrm{v} / \mathrm{v}))$ for seven days and food was removed 24 hours before sacrifice. The rats were anaesthetized with $\mathrm{CO}_{2} / \mathrm{O}_{2}$ and decapitated. The protocol was approved by the institutional ethical review board on experiments with laboratory animals.

\section{Methods}

The livers were isolated and homogenized in ice-cold potassium phosphate buffer $(50 \mathrm{mM}$, containing $22.8 \mathrm{mM}$ potassium chloride and $3.4 \mathrm{mM}$ EDTA, pH 7.4) and centrifuged at $10000 \times \mathrm{g}$ for $20 \mathrm{~min}$ at $4^{\circ} \mathrm{C}$. The supernatant was centrifuged at $65000 \mathrm{xg}$ for $60 \mathrm{~min}$ at $4^{\circ} \mathrm{C}$. The microsomes were washed and resuspended to a concentration of 2 grams liver per milliliter buffer and stored at $-80^{\circ} \mathrm{C}$ until use.

The activity of CYP2E1 was quantified using the CYP2E1 mediated conversion of 4-NP into 4-NC. The amount of 4-NP and 4-NC was determined according to Elbarby et al. [15] and Duescher et al. [16] with a few modifications. The total reaction volume of $200 \mu \mathrm{l}$ contained $20 \mu \mathrm{M} 4-\mathrm{NP}$ in a $50 \mathrm{mM}$ potassium phosphate buffer ( $\mathrm{pH}=7.4)$, and an $\mathrm{NADPH}$-generating system $(3.3 \mathrm{mM} \mathrm{MgCl}, 3.3 \mathrm{mM}$ glucose-6-phosphate, 1.3 mM $\beta$-NADP and 0.4 units/ml glucose-6-phosphate dehydrogenase) and the inhibitor. After five minutes of pre-incubation at $37^{\circ} \mathrm{C}$, the reaction was started by adding the microsomes to the reaction mixture (final concentration of $0.65 \mathrm{mg}$ protein per $\mathrm{ml}$ ). The reaction was terminated after 4 minutes at $37^{\circ} \mathrm{C}$ by the addition of $100 \mu \mathrm{l} 10 \%(\mathrm{w} / \mathrm{v})$ tri-chloro-acetic acid (TCA). After centrifuging, the concentrations of 4-NP and the formed 4-NC in the supernatant were determined by HPLC (reversed phase C18 column, mobile phase 30\% acetonitrile in water). The inhibitory activity of several compounds on the conversion of 4-NP into 4-NC by CYP2E1 was determined. The inhibitory activity of the compounds was expressed as $I C_{50^{\prime}}$ which is the concentration of inhibitor that reduced CYP2E1 activity by half.

\section{Results}

CYP2E1 converts 4-NP into 4-NC according to Michaelis-Menten kinetics $\left(\mathrm{V}_{\max }=0.3 \mu \mathrm{mol}\right.$ 4- NC/min/ mg protein and $\mathrm{K}_{\mathrm{m}}=12.8 \mu \mathrm{M}$, figure 1). Most of the tested compounds were able to inhibit the 4-NP conversion. The inhibition was competitive, only affecting $\mathrm{K}_{\mathrm{m}}$ and not $\mathrm{V}_{\max }$ (figure 2). The primary alcohols, secondary alcohols, ketones and aldehydes showed the strongest inhibition. Although the differences in $I C_{50}$ between these groups of compounds were small, the primary alcohols were the most potent inhibitors tested (figure 3 ). The carboxylic acids had less inhibitory potential (i.e. $I_{50}$ above $200 \mu \mathrm{M}$ with 4-NP concentration of $20 \mu \mathrm{M}$ ).

The length of the alkyl chain greatly affected the inhibitory activity. Increasing the alkyl chain of the substrate resulted in stronger inhibition of CYP2E1 activity. The activity of the carboxylic acids however did not increase up to a chain length of five carbon atoms. From a length of six carbon atoms, the inhibitory activity increased at increasing length of the alkyl chain. 


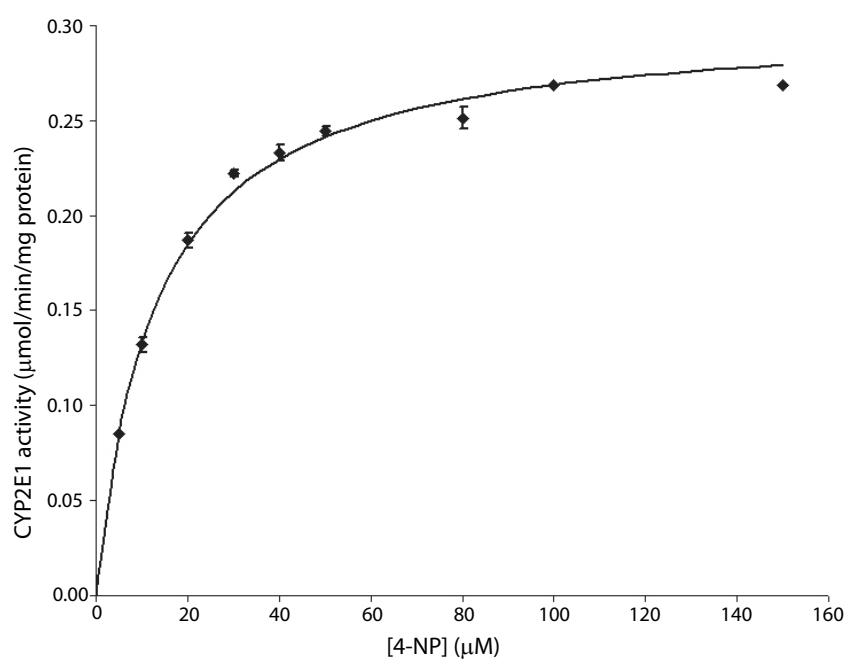

Figure 1: Michaelis-Menten curve of the activity of CYP2E1, with 4-nitrophenol as substrate. The results are expressed as average $( \pm S D, n=3)$.

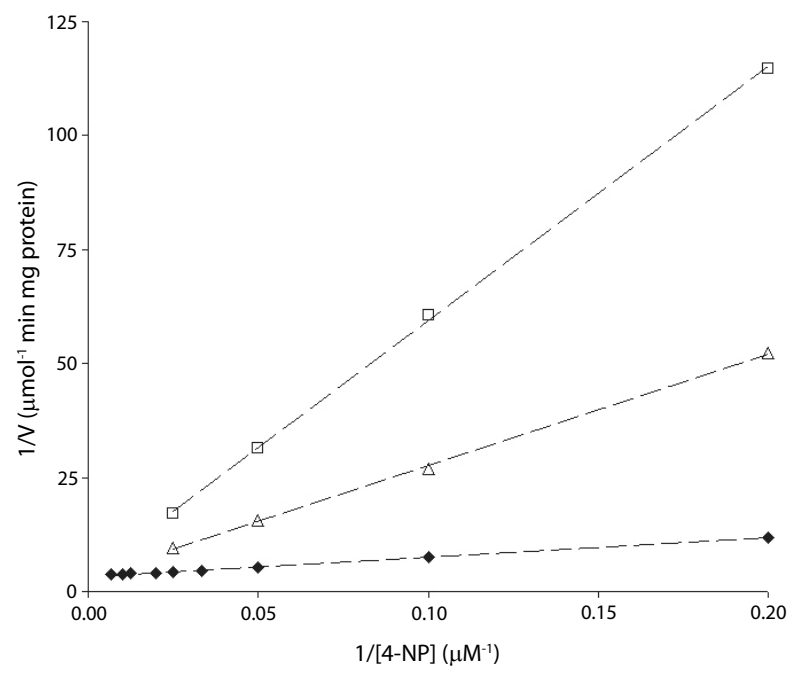

Figure 2: Two examples illustrating the competitive nature of the inhibition of CYP2E1 activity. The inhibition of 1-octanol $(\square, 10 \mu \mathrm{M})$, and 2-butanone $(\triangle, 100 \mu \mathrm{M})$ are depicted in this Lineweaver-Burk plot, together with the control without an inhibitor $(\diamond)$.

The known inducers of CYP2E1, ethanol and propanone (acetone) $[17,18]$, did not show significant inhibition of CYP2E1. 


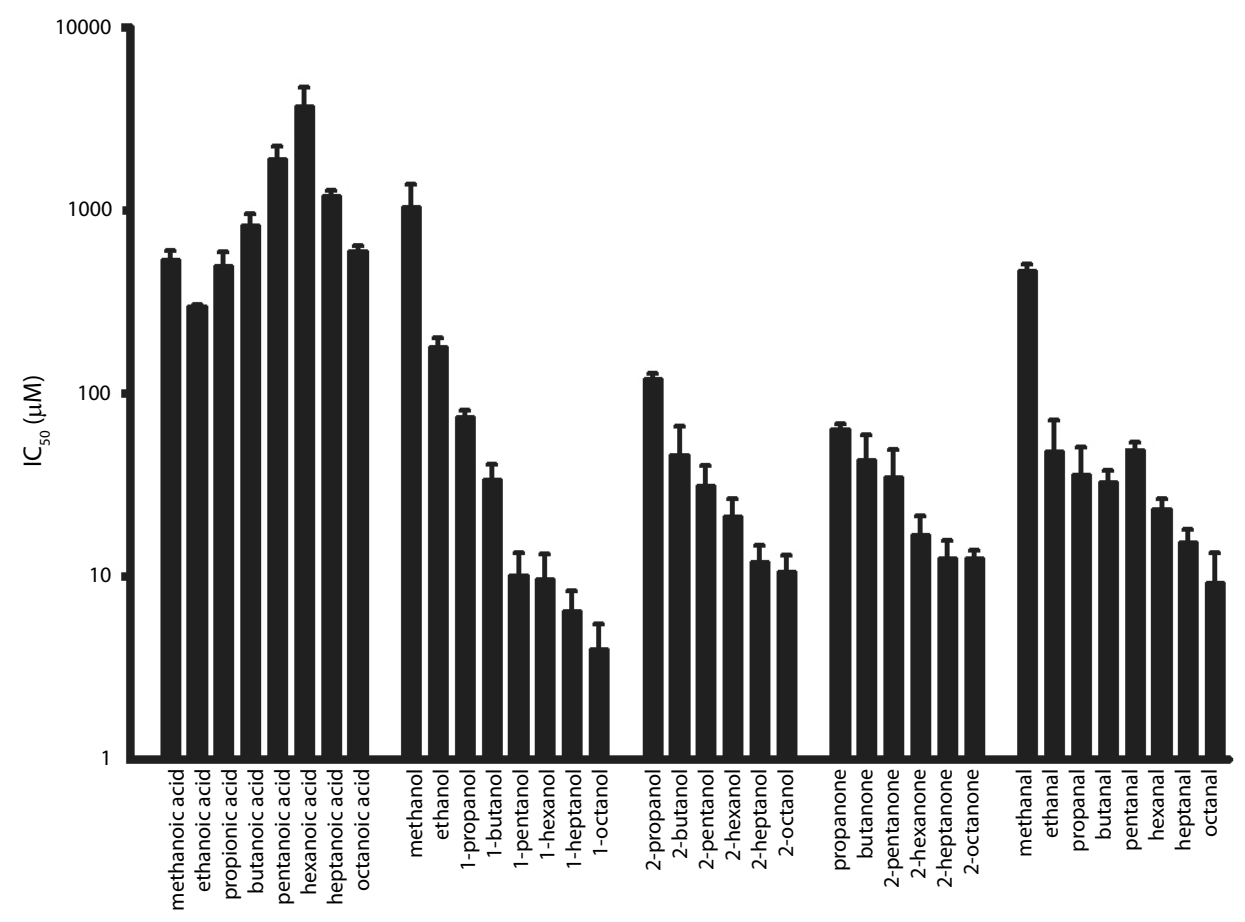

Figure 3: The $I C_{50}$ the concentration of inhibitor that reduces CYP2E1 activity by 50\%, of each tested carboxylic acids, primary alcohols, secondary alcohols, ketones and aldehydes. The results are expressed as mean ( \pm half of the range, $n=2)$. Note the logarithmic scale.

\section{Discussion}

The aim of the present study was to identify the functional requirements of the compounds to obtain inhibition of CYP2E1 activity. To this end, five classes of compounds were tested: primary alcohols, secondary alcohols, ketones, aldehydes and carboxylic acids (small fatty acids).

Surprisingly, it was found that the carboxylic acids only showed weak inhibitory activity, whereas fatty acids are the endogenous substrates of the enzyme. Although these small carboxylic acids (up to eight carbon atoms) could bind to CYP2E1, w-hydroxylation of these small fatty acids is not required in vivo. Other metabolic routes are available for these small carboxylic acids, which decrease the necessity of metabolism of these compounds by CYP2E1.

Homology modeling of CYP2E1 by Collom et al. [19] and protein crystallization by Porubsky et al. [20] showed that the access channel and the active site of CYP2E1 are lipophilic. This weak inhibitory effect of carboxylic acids could be due to the practically complete deprotonation of the carboxylgroup $(-\mathrm{COOH})$ at the $\mathrm{pH}$ of 7.4 ( $\mathrm{pK}_{\mathrm{a}}$ is approximately 4.8). The cavity of the active site is surrounded by lipophilic amino acids, primarily phenylalanine. The charge of the deprotonated carboxylic acids might impede the access to the lipophilic active site of CYP2E1, which could cause the weak inhibitory effect of those compounds. The other classes of compounds tested are not charged at the 
$\mathrm{pH}$ used. For these neutral compounds, the lipophilic character of the access channel is no barrier. This results in a stronger inhibitory effect of the neutral compounds compared with the carboxylic acids.

The potency of inhibition increased at the increasing length of the alkyl chain. There is a correlation between the $\log P$ of the compound (which is also determined by the chain length) and the inhibitory activity (figure 4). The positive correlation between lipophilicity and inhibitory activity is in accordance with previous studies demonstrating that compounds require a certain lipophilic character in order to qualify as an inhibitor for CYP2E1 [21].

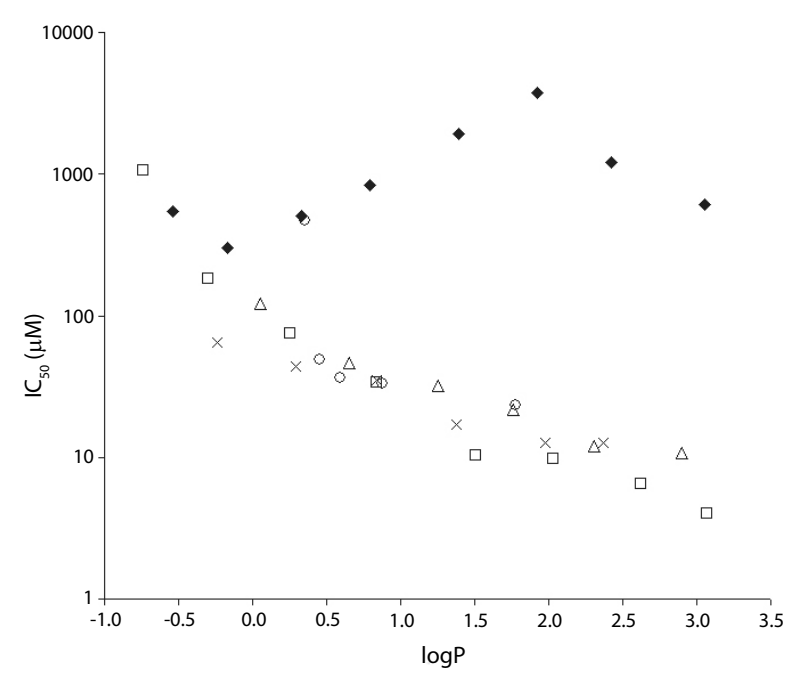

Figure 4: The relation between the lipophilicity of the compound (IogP) and the $I C_{50}$ of CYP2E1 inhibition. The groups of compounds tested are carboxylic acids $(\bullet)$, primary alcohols ( $\square$ ), secondary alcohols $(\Delta)$, ketones $(X)$ and aldehydes ( 0 ).

Interestingly, the carboxylic acids display a different correlation between the logP and the $\mathrm{IC}_{50}$. The $I_{50}$ does not decrease with the logP until a lipophilicity of two (hexanoic acid). Starting from six carbon atoms and longer, the $\mathrm{IC}_{50}$ decreased.

The endogenous substrates for CYP2E1 are the long chain fatty acids. Porubsky et al. [20] suggested that along the access channel, the carboxyl group of the fatty acids interacts with specific positively charged amino acids. This interaction of the negatively charged carboxylic group of the substrate with this positively charged part of the enzyme helps positioning the alkyl chain of the fatty acids correctly into the active site for $\omega$-hydroxylation. The interaction site of the carboxyl group was suggested to be 11 to 15 carbon bond lengths away from the active site. Our data indicates that short chain carboxylic acids up to a chain length of eight carbon atoms could bind to these specific amino acids. From the length of six carbon atoms and longer, the access channel could be blocked by the alkyl chain, preventing the access of 4-NP to the active site. The smaller carboxylic acids (from 
one to five carbon atoms) might also interact. However, their alkyl chain is too short and therefore their affinity to the enzyme too low to prevent access of 4-NP to the active site and inhibit CYP2E1 activity.

Specific inhibition of CYP2E1 will prevent the loose coupling of the substrate and dissociation of the high energetic $\mathrm{Fe}^{2+}-\mathrm{O}_{2} \cdot \mathrm{RH}$ complex and thereby preventing the formation of ROS. In addition, the futile cycling of CYP2E1 is prevented by blocking the active site with an inhibitor. When the formation of ROS is reduced, the oxidative stress (second hit in the development of NASH) will decrease. Less hepatic stellate cells will be activated and the growth of fibrotic tissue will be prevented [22, 23]. If CYP2E1 is inhibited, ultimately the deterioration of the liver function in patients with NAFLD or NASH could be halted. The present study shows that the lipophilicity of a compound is the major determinant for CYP2E1 inhibition in the series tested, and the functional groups tested (except for the carboxylic acids) do not contribute to the inhibitory activity. The inhibition of CYP2E1 activity is primarily determined by the lipophilicity of the substrates. 


\section{References}

1. Angulo, P., Nonalcoholic fatty liver disease. N. Engl. J. Med., 2002. 346(16): p. 1221-1231.

2. Loria, P., et al., Non-alcoholic fatty liver disease (NAFLD) and cardiovascular disease: an open question. Nutr Metab Cardiovasc Dis, 2007. 17(9): p. 684-698.

3. Day, C.P. and James, O.F., Steatohepatitis: a tale of two "hits"? Gastroenterology, 1998. 114(4): p. 842-845.

4. Caldwell, S.H., et al., Mitochondrial abnormalities in non-alcoholic steatohepatitis. J. Hepatol., 1999. 31 (3): p. 430-434.

5. Kallwitz, E.R., McLachlan, A., and Cotler, S.J., Role of peroxisome proliferators-activated receptors in the pathogenesis and treatment of nonalcoholic fatty liver disease. World J. Gastroenterol., 2008. 14(1): p. 22-28.

6. Adas, F., et al., Interspecies variations in fatty acid hydroxylations involving cytochromes P450 2 E1 and 4A. Toxicol. Lett., 1999. 110(1-2): p. 43-55.

7. Amet, Y., et al., Evidence that cytochrome P450 2E1 is involved in the (omega-1)-hydroxylation of lauric acid in rat liver microsomes. Biochem. Biophys. Res. Commun., 1994. 203(2): p. 1168-1174.

8. Zerilli, A., et al., Both cytochromes P450 $2 \mathrm{E} 1$ and 3A are involved in the O-hydroxylation of p-nitrophenol, a catalytic activity known to be specific for P450 2E1. Chem. Res. Toxicol., 1997. 10(10): p. 1205-1212.

9. Chtioui, $\mathrm{H}_{\text {., }}$ et al., Expression and activity of the cytochrome P450 $2 \mathrm{E} 1$ in patients with nonalcoholic steatosis and steatohepatitis. Liver Int, 2007. 27(6): p. 764-771.

10. Robertson, G., Leclercq, I., and Farrell, G.C., Nonalcoholic steatosis and steatohepatitis. II. Cytochrome P-450 enzymes and oxidative stress. Am J Physiol Gastrointest Liver Physiol, 2001. 281(5): p. G1135-1139.

11. Weltman, M.D., et al., Hepatic cytochrome P450 $2 \mathrm{E} 1$ is increased in patients with nonalcoholic steatohepatitis. Hepatology, 1998. 27(1): p. 128-133.

12. Guengerich, F.P. and Johnson, W.W., Kinetics of ferric cytochrome P450 reduction by NADPH-cytochrome P450 reductase: rapid reduction in the absence of substrate and variations among cytochrome P450 systems. Biochemistry (Mosc). 1997. 36(48): p. 14741-14750.

13. Koop, D.R., Chernosky, A., and Brass, E.P., Identification and induction of cytochrome P450 $2 E 1$ in rat Kupffer cells. J. Pharmacol. Exp. Ther., 1991. 258(3): p. 1072-1076.

14. Ronis, M.J., et al., Expression and distribution of cytochrome P450 enzymes in male rat kidney: effects of ethanol, acetone and dietary conditions. Biochem. Pharmacol., 1998. 55(2): p. 123-129.

15. Elbarbry, F., Wilby, K., and Alcorn, J., Validation of a HPLC method for the determination of p-nitrophenol hydroxylase activity in rat hepatic microsomes. J. Chromatogr. B Analyt. Technol. Biomed. Life. Sci., 2006. 834(1-2): p. 199-203.

16. Duescher, R.J. and Elfarra, A.A., Determination of p-nitrophenol hydroxylase activity of rat liver microsomes by high-pressure liquid chromatography. Anal. Biochem., 1993. 212(2): p. 311-314.

17. Robin, M.A., et al., Ethanol increases mitochondrial cytochrome P450 $2 E 1$ in mouse liver and rat hepatocytes. FEBS Lett., 2005. 579(30): p. 6895-6902.

18. Lu, Y., et al., Cytochrome P450 $2 E 1$ contributes to ethanol-induced fatty liver in mice. Hepatology, 2008. 47(5): p. 1483-1494.

19. Collom, S.L., et al., CYP2E1 active site residues in substrate recognition sequence 5 identified by photoaffinity labeling and homology modeling. Arch. Biochem. Biophys., 2007. 459(1): p. 59-69.

20. Porubsky, P.R., Meneely, K.M., and Scott, E.E., Structures of human cytochrome P-450 2E1. Insights into the binding of inhibitors and both small molecular weight and fatty acid substrates. J. Biol. Chem., 2008. 283(48): p. 33698-33707.

21. Lewis, D.F., Essential requirements for substrate binding affinity and selectivity toward human CYP2 family enzymes. Arch. Biochem. Biophys., 2003. 409(1): p. 32-44.

22. Nieto, N., Friedman, S.L., and Cederbaum, A.I., Stimulation and proliferation of primary rat hepatic stellate cells by cytochrome P450 2E1-derived reactive oxygen species. Hepatology, 2002. 35(1): p. 62-73.

23. Poli, G., Pathogenesis of liver fibrosis: role of oxidative stress. Mol. Aspects Med., 2000. 21(3): p. 49-98. 
$d x_{1}=-k_{j} x_{1}$
$d x_{2}=k_{2} x_{1}$ 


\section{Chapter 7}

\section{Difference in effect of apples, fruit salad and fruit beverage on uric}

acid concentration and total plasma antioxidant capacity.

Jiska M. Balk, Guido R.M.M. Haenen, Saskia J. Rietjens, Bertine J. Philipsen-Geerling, Ger H. Koek, Aalt Bast 


\section{Abstract}

The intake of fruit and vegetables is associated with a positive health effect. This health benefit has been linked to the antioxidant content of fruit. In a randomized trial, the effect of the intake of three different fruit servings on the plasma total antioxidant capacity was compared in twenty-four healthy volunteers that had a low antioxidant diet for one day. The change in Trolox Equivalent Antioxidant Capacity and uric acid concentration after intake was measured for six hours after intake. The effect of apples on the antioxidant capacity is primarily due to the uric acid concentration, an effect induced by fructose. The fructose effect with apples could not prevent a decrease in plasma total antioxidant capacity. With the fruit salad or the fruit beverage, the fructose effect was relatively low, but antioxidants delivered by the fruit intake prevented the decrease of the plasma total antioxidant capacity. This contribution of antioxidants is comparable for the fruit beverage and the fruit salad. 


\section{Introduction}

The intake of fruit and vegetables is believed to provide a health benefit. Several studies have shown that an adequate consumption of fruit and vegetables reduces the risk of cancer [1] and coronary heart disease [2]. These diseases involve the formation of reactive oxygen species, which can damage essential proteins, lipids and the DNA [3,4]. This damage can be prevented by antioxidants that protect against these reactive oxygen species.

In general, fruit is an abundant source of antioxidants [5]. The neutralization of the reactive oxygen species has been implicated in the health benefit. In the present study, the effect of three kinds of fruits servings on the antioxidant capacity of blood plasma was compared. Lotito and Frei [6] have stated that the effect of fruit on the antioxidant capacity is predominantly due to a rise in the uric acid concentration instead of the antioxidants present in fruit. The rise in uric acid was ascribed to the fructose present in the fruit. In the present study, the effect of different fruit servings on the uric acid as well as the total plasma antioxidant capacity was compared.

Three kinds of fruit servings were compared. i) A fruit salad, which represented the ten most consumed fruits in the Netherlands in a dose proportional to their relative consumption. ii) A fruit beverage, because several fruit based drinks have been introduced to the market as a daily portion of fruit. iii) Apples as a reference fruit serving, because the study of Lotito and Frei [7] was based on the intake of apples.

Before the intake of the fruit serving, the volunteers were restricted to an antioxidant low diet for one day, in order to minimize individual variation and to increase the sensitivity of the study. It was found that apples indeed predominantly affected uric acid. The effect of the fruit salad and the fruit beverage on the total plasma antioxidant capacity was however not predominantly due to the uric acid concentration.

\section{Materials and Methods}

\section{Chemicals}

2,2'-azinobis(3-ethylbenzthiazoline-6-sulfonic acid) (ABTS) and uric acid were obtained from Sigma Chemical Co., St. Louis, USA. 2,2'-azino-bis(2-amidinopropane) dihydrochloride (ABAP) was obtained from Brunschwig Chemicals, Amsterdam, The Netherlands. Trolox was obtained from Aldrich Chemical, Steinheim, Germany. All other chemicals were of analytical purity.

\section{Subjects and study design}

The study was approved by the Medical Ethical Review Board of Maastricht University and registered in an international clinical trial register (clinicaltrials.gov NCT00545415). Written informed consent was obtained from each volunteer. Only non-smoking males were included in this study. The volunteers did not use any medication, were not involved in top-sport, had an alcohol consumption of less than three glasses each day and did not donate blood the last six months before the start of the study. 
Each volunteer had three different interventions, the intake of fruit salad, apples or a fruit beverage, as a one-time large dose. The different intakes were assigned in random order and each volunteer acted as his own control. A washout period of one week was used between each intervention. One day before the intervention, the volunteers had a strict diet, which was low in antioxidants and they received a standardized dinner. The diet was abstained from fruit, vegetables, chocolate, tea, alcohol, olives, olive oil, antioxidant containing supplements and other food and drinks that contained those components. The volunteers had to keep a food diary in order to monitor the compliance of the diet. A fasted blood sample was taken as a baseline value. Blood samples were taken at 10, 20, 30, 45, $60,75,90,120,150,180,210,240,300$ and 360 minutes after intake of the fruit serving, in order to determine the kinetics of the antioxidant capacity in blood plasma. During these hours, the volunteers were abstained from food and drinks except water. Each individual completed the study (i.e. the three interventions) within four weeks. The whole study was finished within six months.

The expected standard deviation $(\sigma)$ of the plasma Trolox Equivalent Antioxidant Capacity (TEAC) was 5\% [8-10]. The expected effect ( $\delta$ ) was an increase in antioxidant capacity of $4 \%$ [10]. The number of volunteers needed is calculated according to L. Sachs:

$N=2\left(z_{\alpha / 2}-z_{\pi}\right)^{2} *(\sigma / \delta)^{2}=15.7 *(5 / 4)^{2}=24.53 \quad$ Equation 1

\section{Fruit servings}

The apple intervention was the consumption of 400 grams of peeled apple (Golden Delicious). The fruit salad (400 grams) contained the ten most consumed fruits in the Netherlands, according to their percentages in the NEVO chart (abbreviation of Netherlands Food Composition) of 2006 (table 1). The fruit beverage Fruit 2 day $^{\oplus}$ (strawberry/orange, $2 \times 200 \mathrm{~mL}$ ) is a registered product from HERO Nederland B.V.. The fruit beverages were provided by HERO Nederland B.V. and stored at $4^{\circ} \mathrm{C}$ until use. The apples and components of the fruit salad were bought freshly at the supermarket and stored at $4^{\circ} \mathrm{C}$ until use.

The total fructose content (free fructose and fructose from sucrose) of Fruit2day ${ }^{\oplus}$ was $5.6 \%(\mathrm{w} / \mathrm{w})$. The total fructose content of apples and the fruit salad were $7.6 \%(\mathrm{w} / \mathrm{w})$ and $6.3 \%(\mathrm{w} / \mathrm{w})$, respectively.

Table 1: Popularity of fruit consumed in the Netherlands 2006

$\begin{array}{ll}\text { Fruit } & \text { Percentage } \\ \text { Apple } & 19.4 \\ \text { Apple (peeled) } & 17.7 \\ \text { Banana } & 14.9 \\ \text { Orange } & 14.1 \\ \text { Mandarin } & 8.8 \\ \text { Pear } & 7.8 \\ \text { Strawberry } & 3.4 \\ \text { Grapefruit (white) } & 2.6 \\ \text { Kiwi } & 2.6 \\ \text { Melon } & 2.0\end{array}$


Analysis

Blood samples $(4 \mathrm{~mL})$ were taken in heparinized tubes and centrifuged immediately (1000g for 5 min at $4{ }^{\circ} \mathrm{C}$ ) to obtain the blood plasma. The blood plasma was stored at $-80^{\circ} \mathrm{C}$ until analysis. The plasma was deproteinized by the addition of 10\% trichloroacetic acid to the plasma in the ratio 1:1. The TEAC was determined in deproteinized plasma according to Fischer et al. [8]. The concentration of uric acid in deproteinized plasma was determined with HPLC as described by Rietjens et al. [11]. The uric acid subtracted-TEAC was calculated according to Boots et al. [12]. The samples of each volunteer for all the three interventions were analyzed in one day. The analysis of all the samples was performed within six months.

\section{Outcomes}

The primary outcome of the study was the effect of the fruit servings on the plasma total antioxidant capacity. It was expected that the in vivo effect of the fruit beverage is equivalent to that of the fruit salad. The apples were expected to give the lowest increase in TEAC, based on their lower antioxidant content.

\section{Statistical analysis}

The difference of the TEAC and uric acid concentrations from baseline was calculated. Statistics were performed with a two-tailed paired t-test with $a=0.05$. Statistics of each fruit serving was performed for the difference from baseline levels. Linear regression analysis of the TEAC and the uric acid concentration was performed by the least squares method.

\section{Results}

Twenty-five male volunteers were included in this study. One volunteer withdrew his participation during the study and the samples of this volunteer were not analyzed. The twenty-four volunteers that completed the study had an average age of 30 years (SD 10) and an average body mass index of $23.6 \mathrm{~kg} \cdot \mathrm{m}^{-2}$ (SD 2.7). All volunteers complied with the dietary restrictions. The fruit serving was supplied in the morning and the change in TEAC in blood plasma was followed for the next six hours. No side effects were reported by the volunteers.

The plasma TEAC decreased in time after intake of the fruit servings (figure 1). No difference was found in the level of decrease of the TEAC between apples, fruit salad or fruit beverage.

The plasma uric acid concentration (figure 2) also decreased after intake of fruit beverage or fruit salad. After consumption of apples, a delay in the onset of the decrease was found.

A strong correlation was found between TEAC and the uric acid concentration in plasma (correlation coefficient of $0.8, P<<0.01$, figure 3 ). The slope of the regression line is 0.9 , which is almost equal to the TEAC of uric acid. The TEAC of uric acid is 1.0, which means that $1 \mu \mathrm{mol} / \mathrm{L}$ uric acid represents 1 $\mu \mathrm{mol}$ trolox equivalent [13].

The contribution of plasma antioxidants other than uric acid can by calculated by subtracting the 
uric acid contribution from the TEAC. A clear difference was found between the apples, the fruit salad and the fruit beverage on this uric acid-subtracted TEAC (figure 4). The uric acid-subtracted TEAC after intake of apples decreased in time, while the uric acid subtracted-TEAC after intake of fruit salad and fruit beverage remained constant.

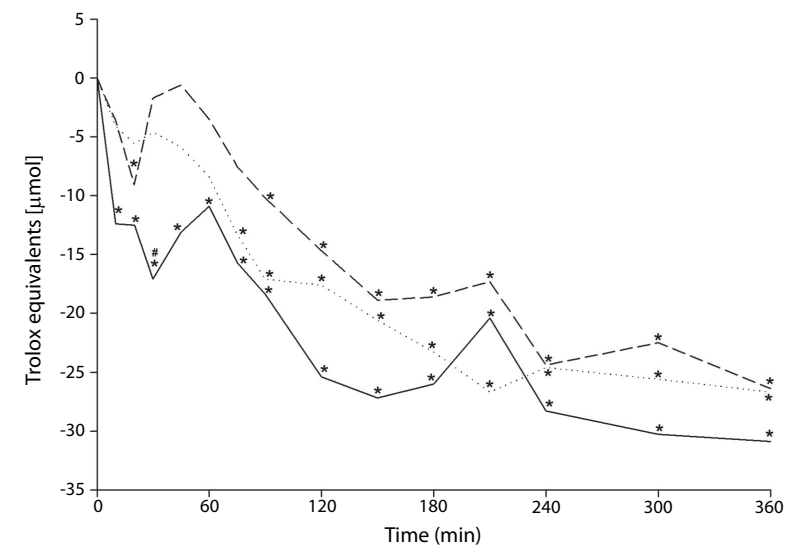

Figure 1. The effect of fruit servings on the plasma TEAC. The average TEAC (normalized to the baseline of 566.5 Trolox Equivalents) of the twenty-four volunteers was determined after intake of the fruit beverage (solid line), fruit salad (longdash line) and apples (small-dash line). Statistical analysis showed significant differences to baseline levels (.) $(P<0.05)$, significant differences between apples and the fruit beverage $(\#)(P<0.05)$ and significant differences between apples and fruit salad $(+)(P<0.05)$. There was no significant difference between the fruit salad and the fruit beverage.

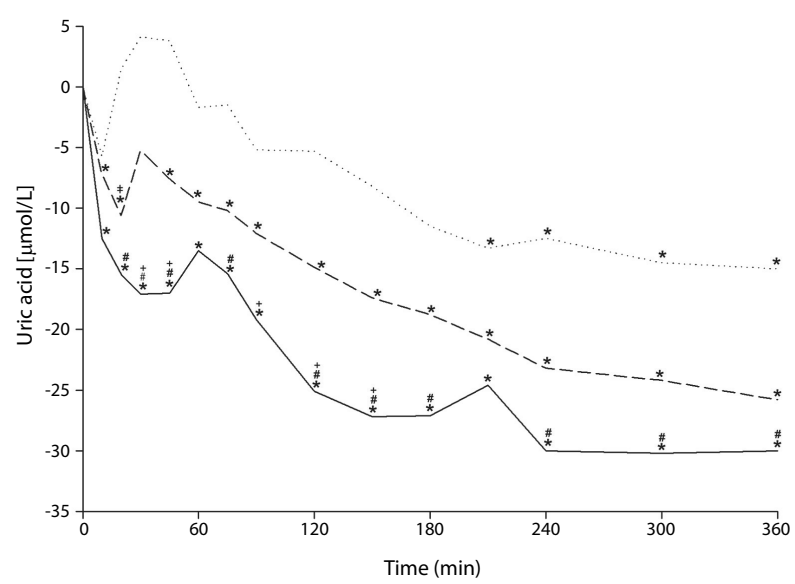

Figure 2. The effect of fruit servings on the plasma uric acid concentration. The average uric acid concentration (normalized to the baseline of 290.1 umol/L uric acid) of the twenty-four volunteers was determined after intake of fruit beverage (solid line), fruit salad (long-dash line) and apples (small-dash line). Statistical analysis showed significant differences to baseline levels (.) $(P<0.05)$, significant differences between apples and fruit beverage $(\#)(P<0.05)$, significant differences between apples and fruit salad $(+)(P<0.05)$ and significant differences between fruit beverage and fruit salad $(\neq)(P<0.05)$. 


\section{Discussion}

The effect on the plasma total antioxidant capacity after intake of three different fruit servings was compared. Instead of an expected increase, both the total antioxidant capacity of plasma and the uric acid concentrations decreased during six hours after intake of the fruit servings (figure 1 and 2). Uric acid has a half life of about 20 hours in plasma [14], which would result in an elimination of around $18 \%$ during the six hours of the experiment. The decrease of uric acid (figure 2 ) was only 5.2 $\%, 8.9 \%$ and $10 \%$ after intake of apples, fruit salad and fruit beverage, respectively. Since fruit does not contain uric acid, this indicates that some uric acid has to be produced. Uric acid is normally produced by metabolism and especially during exercise via ATP breakdown [9]. The volunteers were relatively inactive during the study, which rules out exercise as a major source of uric acid production. Uric acid production could also be due to fructose uptake. As described by Lotito and Frei [6], fructose is rapidly converted in the liver into fructose-1-phosphate, which leads to a decrease in ATP and inorganic phosphate. ATP and inorganic phosphate are inhibitors of 5'-nucleotidase and AMP deaminase, which are needed for the degradation of AMP to uric acid. The absence of the inhibitors for these enzymes leads to an increased breakdown of AMP via inosine to uric acid (reviewed in Mayes et al. [15]). This fructose effect leads to an increased production of uric acid. An increase in plasma uric acid concentration after 90 minutes was seen if the dose of fructose is above a threshold of $0.5 \mathrm{~g}$ per $\mathrm{kg}[16]$.

Apples contain relatively high levels of fructose (7.6\%) that is comparable to the threshold of $0.5 \mathrm{~g}$ fructose per kg. Indeed no decrease in uric acid concentration after intake of apples was observed after 90 minutes. The fruit beverage and the fruit salad, which contained less fructose, showed a decrease in uric acid levels after 90 minutes. Also after giving apples, a decrease in uric acid levels was found at the end of our study (figure 2). This decrease is due to the clearance of uric acid from the body. The fact that with $0.5 \mathrm{~g} / \mathrm{kg}$ fructose after 90 minutes no change in uric acid levels is found, is caused by the fructose-induced production of uric acid that compensates the clearance. With a lower fructose intake, the production cannot compensate for the clearance of uric acid.

From literature, it is known that uric acid is a strong endogenous antioxidant in plasma $[17,18]$. It efficiently scavenges several reactive oxygen species (singlet oxygen, hydroxyl radicals) and oxygen derived radicals (oxo-heme oxidants, peroxyl radicals) [18]. The TEAC method measures the total antioxidant capacity, of which a large part is due to uric acid. The strong correlation between the uric acid concentrations and the TEAC value (figure 3 ) also indicates that uric acid is a major contributor to the plasma total antioxidant capacity. A high TEAC of plasma is accompanied with a high uric acid concentration, for most individuals as well as on group level.

The absolute contribution of the antioxidants can be discriminated from the fructose effect by subtracting the uric acid contribution to the TEAC [12] (figure 4). After intake of apples, the plasma antioxidants other than uric acid decreased. Apparently, the apples did not provide sufficient antioxidants to prevent this decrease in plasma antioxidants. After intake of fruit beverage or fruit salad, this uric acid-subtracted TEAC did not decrease. 


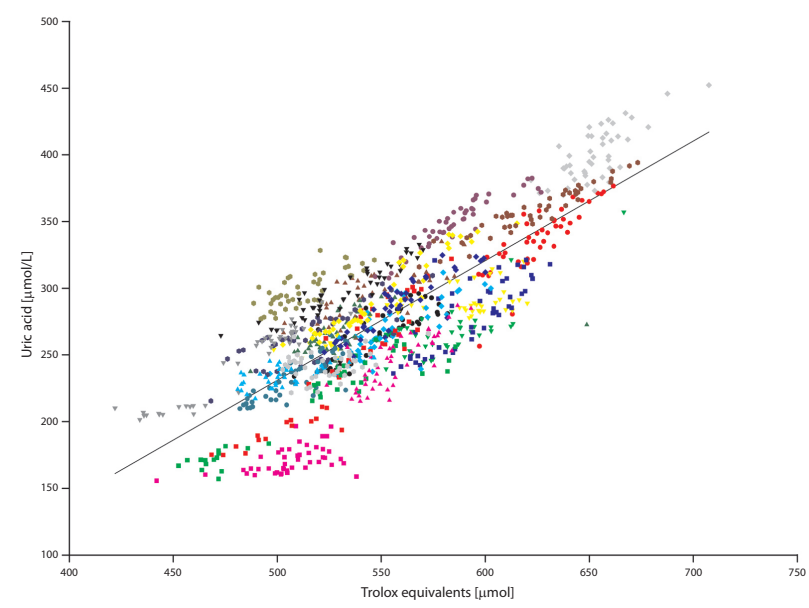

Figure 3. The correlation between the TEAC and uric acid concentration. Each unique combination of colour and shape represents the data of one volunteer (three interventions, fifteen samples for each intervention). The correlation coefficient is $0.8(P<<0.01)$.

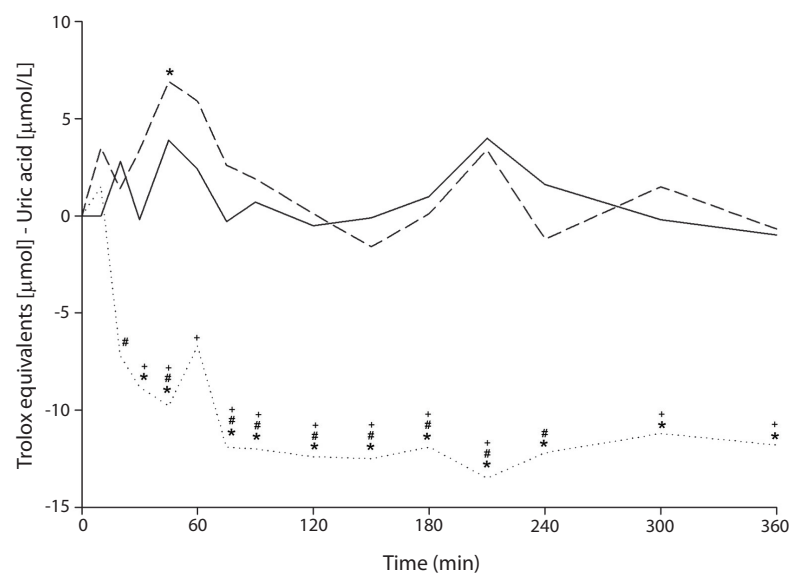

Figure 4. The effect of intake of fruit beverage (solid line), fruit salad (long-dash line) and apples (small-dash line) on the uric acid-subtracted TEAC. The data was normalized to baseline and averaged. Statistical analysis showed significant differences to baseline levels (.) $(P<0.05)$, significant differences between apples and fruit beverage $(\#)(P<0.05)$ and significant differences between apples and fruit salad $(+)(P<0.05)$. There was no significant difference between the fruit salad and the fruit beverage. 
The antioxidants taken up from these fruit servings apparently prevented this decrease in antioxidant capacity of plasma.

Health organizations advise to have a sufficient intake of fruit. The observed in vivo differences between the three fruit servings found in the present study indicate that one has to discriminate between different types of fruit.

The present study confirms that the intake of fruit directly affects the uric acid production, induced by fructose. With apples, this fructose effect prevails. With the fruit salad or the fruit beverage, the fructose effect was low, and antioxidants delivered by the fruit intake prevented the decrease of the plasma total antioxidant capacity. This contribution of antioxidants is comparable for the fruit beverage and the fruit salad. 


\section{Acknowledgements}

We would like to thank M.J.Drittij for her valuable technical assistance. This study was financially supported by HERO Nederland B.V.. 


\section{References}

1. Riboli, E. and Norat, T., Epidemiologic evidence of the protective effect of fruit and vegetables on cancer risk. Am. J. Clin. Nutr., 2003. 78(3 Suppl): p. 559S-569S.

2. He, F.J., et al., Increased consumption of fruit and vegetables is related to a reduced risk of coronary heart disease: meta-analysis of cohort studies. J. Hum. Hypertens., 2007. 21(9): p. 717-728.

3. Davies, M.J., The oxidative environment and protein damage. Biochim. Biophys. Acta, 2005. 1703(2): p. 93109.

4. Evans, M.D., Dizdaroglu, M., and Cooke, M.S., Oxidative DNA damage and disease: induction, repair and significance. Mutat. Res., 2004. 567(1): p. 1-61.

5. Paganga, G., Miller, N., and Rice-Evans, C.A., The polyphenolic content of fruit and vegetables and their antioxidant activities. What does a serving constitute? Free Radic. Res., 1999. 30(2): p. 153-162.

6. Lotito, S.B. and Frei, B., The increase in human plasma antioxidant capacity after apple consumption is due to the metabolic effect of fructose on urate, not apple-derived antioxidant flavonoids. Free Radic. Biol. Med., 2004. 37(2): p. 251-258.

7. Lotito, S.B. and Frei, B., Relevance of apple polyphenols as antioxidants in human plasma: contrasting in vitro and in vivo effects. Free Radic. Biol. Med., 2004. 36(2): p. 201-211.

8. Fischer, M.A., et al., Determination of the antioxidant capacity in blood. Clin. Chem. Lab. Med., 2005. 43(7): p. 735-740.

9. Rietjens, S.J., et al., A single session of resistance exercise induces oxidative damage in untrained men. Med Sci Sports Exerc, 2007. 39(12): p. 2145-2151.

10. van den Berg, R., et al., A vegetable/fruit concentrate with high antioxidant capacity has no effect on biomarkers of antioxidant status in male smokers. J. Nutr., 2001. 131(6): p. 1714-1722.

11. Rietjens, S.J., Bast, A., and Haenen, G.R.M.M., New insights into controversies on the antioxidant potential of the olive oil antioxidant hydroxytyrosol. J. Agric. Food Chem., 2007. 55(18): p. 7609-7614.

12. Boots, A.W., et al., In vitro and ex vivo anti-inflammatory activity of quercetin in healthy volunteers. Nutrition, 2008. 24(7-8): p. 703-710.

13. Rice-Evans, C. and Miller, N., Measurement of the antioxidant status of dietary constituents, low density lipoproteins and plasma. Prostaglandins Leukot. Essent. Fatty Acids, 1997. 57(4-5): p. 499-505.

14. Geren, W., et al., The fate of uric acid in man. J. Biol. Chem., 1950. 183(1): p. 21-31.

15. Mayes, P.A., Intermediary metabolism of fructose. Am. J. Clin. Nutr., 1993. 58(5 Suppl): p. 754S-765S.

16. Heuckenkamp, P.U. and Zollner, N., Fructose-induced hyperuricaemia. Lancet, 1971. 1(7703): p. 808-809.

17. Becker, B.F., Towards the physiological function of uric acid. Free Radic. Biol. Med., 1993. 14(6): p. 615-631.

18. Ames, B.N., et al., Uric acid provides an antioxidant defense in humans against oxidant- and radical-caused aging and cancer: a hypothesis. Proc. Natl. Acad. Sci. U. S. A., 1981. 78(11): p. 6858-6862. 
$d x_{1}=-k_{j} \cdot x_{1}$
$d x_{2}=k_{0} w_{1}$ 
Chapter 8

\section{Discussion}
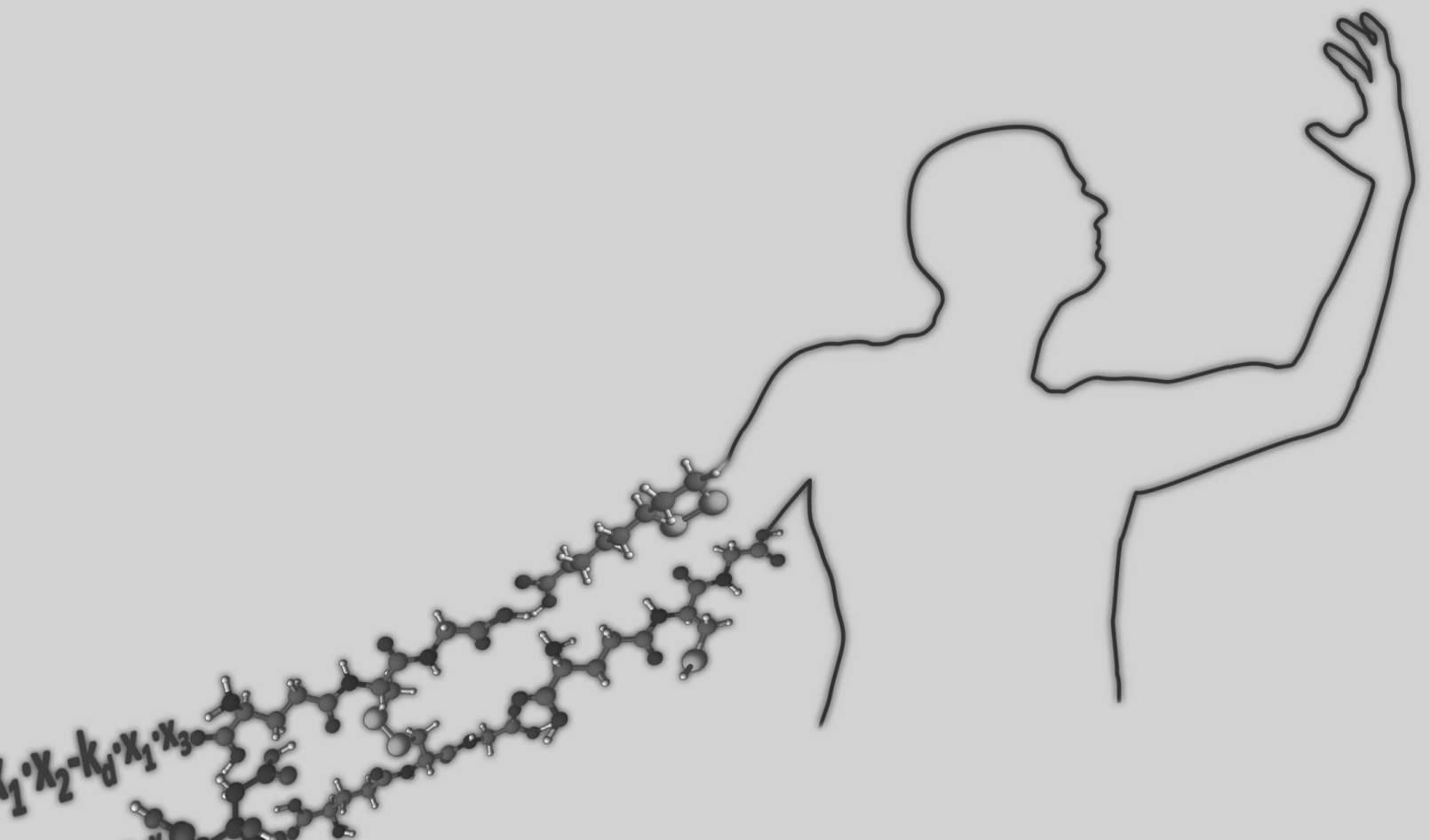

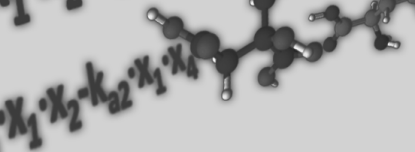



Molecular oxygen is indispensable to life. It is used by several enzymes to produce reactive oxygen species as part of physiological processes varying from inflammation response to the control of vascular function. The produced reactive oxygen species could however also damage proteins, lipid membranes and DNA, resulting in dysfunction of the cellular system, which could be the onset of many diseases.

Damage by reactive oxygen species can be prevented by antioxidants. This prevention can be achieved in different ways: scavenging of the reactive species, inhibition of oxidative enzymes or scavenging of precursors of reactive species.

The scavenging potential of compounds is usually screened in competition assays, in which the antioxidant has to compete with a detector molecule for the reactive species. The extent of protection of the detector by the antioxidant reflects the antioxidant activity. Protection by the antioxidant is observed when the reaction rate constant of the reaction of the reactive species with the antioxidant $\left(k_{a}\right)$ is higher than that of the reaction with the detector $\left(k_{d}\right)$. The antioxidant activity is often expressed as the reaction rate constant $k_{a}$. In the evaluation of competition assays, it is generally assumed that the concentrations of the antioxidant and the detector do not alter during the assay. The assay is based on the consumption of the detector, which indicates that the concentration of the reactants will change. Computer simulation of competition assays showed that the decrease in concentration of the antioxidant and the detector often greatly affects the observed antioxidant activity. Due to a difference in reaction rate constants, the detector and the antioxidant are consumed in relative different amounts. The resulting change in ratio of concentration of both reactants causes a deviation of the observed antioxidant activity from the theoretical activity, which is based solely on the reaction rate constants. In many assays, the deviation exceeds 100 percent from the actual antioxidant activity. The overall result is that the differences in activity between antioxidants are underestimated and the activity of antioxidants shifts towards the activity of the detector (chapter 2).

This deviation can be diminished by choosing a detector that has a $k_{d}$ comparable to $k_{a}$. In addition, the concentration of reactive species should be as low as possible. An accurate antioxidant activity might be obtained in competition assays using this strategy.

Besides the dynamics, several other aspects of the competition assay need to be critically evaluated. For example, the reactions of reactive species are un-specific and not limited to the antioxidant or the detector alone. The high intrinsic reactivity of the reactive species indicates that additional reactions are likely to occur with other compounds present in the incubation mixture. The antioxidant has to compete for the reactive species not only with the detector, but also with these unspecific additional reactions. Neglecting these additional reactions result in a systematic underestimation of the observed antioxidant activity. This underestimation can easily be prevented by first identifying the relative contribution of these unspecific reactions and the use of an adjusted Stern-Volmer equation to calculate the reaction rate constant of the antioxidant with the reactive species (chapter 3). 
Another critical aspect of competition assays is that in the calculation of $k_{a^{\prime}}$ it is tacitly assumed that the stoichiometry of the reaction with the reactive species with the antioxidant equals one. However, the stoichiometry of the reaction of antioxidants does not have to be one as demonstrated for several $\mathrm{HOCl}$ scavengers. When the actual stoichiometry of an antioxidant is above one, the calculated reaction rate constant is greatly underestimated.

Once a reactive species have been scavenged by an antioxidant, the antioxidant is consumed and is converted into a reaction product. It is not taken into account that this oxidation product is also able to react with the reactive species. So the parent antioxidant as well as the product might act as an antioxidant. At high concentrations of reactive species, this oxidation product accumulates and might contribute to the protection of the detector by scavenging the reactive species. Detailed evaluation of several hypochlorous acid scavenging assays (chapter 4) revealed that the observed antioxidant activity is greatly affected by the activity of the oxidation products formed. Our results demonstrate that the observed activity of $\mathrm{HOCl}$ scavengers is not primarily governed by the reaction rate constant of the antioxidant with the reactive species. The antioxidant activity is best described by the observed capacity; the average number of reactive species scavenged by one molecule of antioxidant, including that of the antioxidant products at $\mathrm{EC}_{50}$ conditions.

In chapters 2 to 4 , it is shown that antioxidant activity determined in vitro is greatly influenced by the experimental conditions used. It should be realized that the aspects that determine the antioxidant activity in a competition assay, also influence the antioxidant activity in vivo. In vitro assays should focus on the primary goal of an antioxidant; the protection of the biological target from oxidation. Therefore, a competition assay should mimic the in vivo situation with respect to the concentrations of the reactants and reaction rate constant of the detector with the reactive species. The description of the antioxidant activity should include, besides the $\mathrm{k}_{\mathrm{a}^{\prime}}$ the contribution of the oxidation products to the protection of the detector.

For example, in burst of locally formed $\mathrm{HOCl}$, the concentration of glutathione is e.g. relatively low, indicating that the sulfoxides and disulfides formed also react with $\mathrm{HOCl}$. The oxidation products will considerably contribute to the protection of the biological target. Further away from the burst or when $\mathrm{HOCl}$ formation is less vigorous, the products do not accumulate in significant amounts and subsequently their protective effect will be negligible.

The in vivo activity of antioxidants is even more complex. An antioxidant does not act in isolation. Antioxidants co-operate and form in vivo an intricate network that also involves regeneration of the product. A classical example is the co-operation between the antioxidants vitamin $\mathrm{C}$ and vitamin $\mathrm{E}$. Both antioxidants have scavenging activity, but vitamin $C$ can also regenerate the oxidized vitamin $E$ to its original reduced form. A co-operation takes place between many antioxidants and can occur via several mechanisms. Lipophilic and hydrophilic antioxidants synergistically protect biological targets from oxidation, which is illustrated in figure 1 for peroxyl radicals present in plasma. This co-operation between the different antioxidants and their oxidized products to provide protection of the biological target is not covered in the screening of single compounds for their scavenging antioxidant activity. 


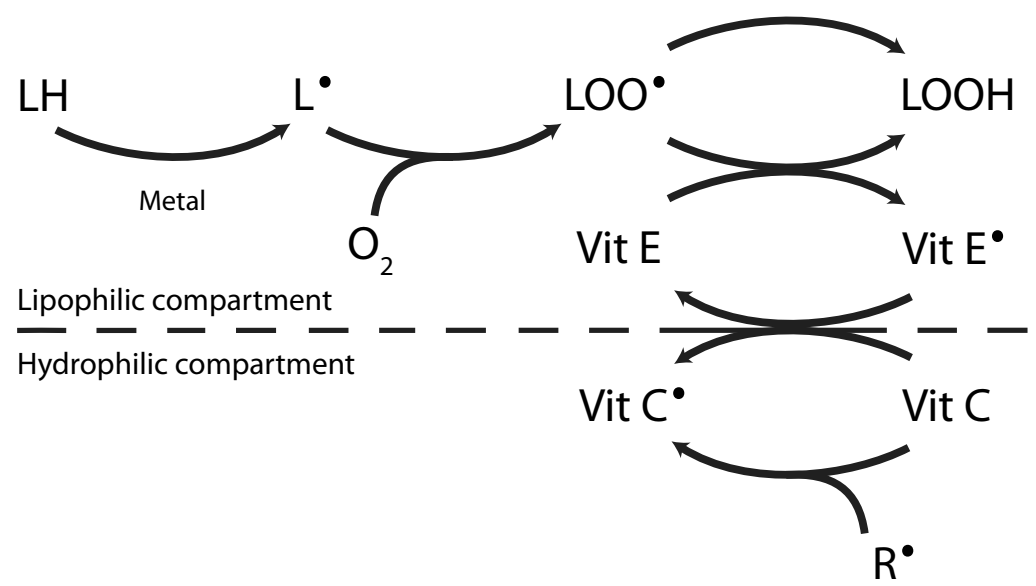

Figure 1. An example of co-operation between antioxidants located in the hydrophilic and lipophilic compartments of plasma. A radical is indicated with a dot. $\mathrm{LH}$ is a lipid molecule and $\mathrm{LOOH}$ is its peroxide.

Although antioxidants could protect the biological target by scavenging the reactive species, it would also be beneficial to prevent the excessive production of reactive species. An unbalanced production of reactive species finally results in dysfunction of the cell, which could be the onset of diseases. In non-alcoholic steatohepatitis (NASH), one specific P450 enzyme present in the liver is upregulated: CYP2E1. CYP2E1 is involved in the fatty acid metabolism and detoxification of compounds. This enzyme is however prone to produce superoxide anion radicals. The exposure of cells to high levels of fatty acids, a feature found in NASH patients, resulted in increased citrate concentrations in the medium of cells. In accordance to these results plasma citrate concentrations were elevated in NASH patients. The molecular structure of citrate (a highly oxidized molecule) suggests that binding to the haem of CYP2E1 will not result in citrate oxidation, but it could enhance the production of superoxide by CYP2E1. However, citrate did not directly increase the superoxide production by CYP2E1. Apparently, induction of superoxide production by microsomal CYP2E1 by citrate is not involved in the second hit in the development of NASH (chapter 5). Further research is necessary to determine the effect of high citrate levels on the cellular function and the induction of CYP2E1.

Since CYP2E1 is responsible for the production of superoxide, antioxidants might protect against superoxide by inhibiting the CYP2E1 mediated superoxide production. In the process to identify effective and specific inhibitors of CYP2E1, the functional requirements of the inhibitors have to be identified. In a series of homologous compounds, the major determinant for the inhibitory potential of compounds appeared to be the lipophilicity (chapter 6). This approach identifies specific inhibitors that could lead to pharmacotherapeutic intervention by antioxidants in the treatment of NASH.

To protect against oxidative stress, antioxidant treatment of diseases is possible if the intake of antioxidants results in an increase in antioxidant content in the human body. Fruit and vegetables contain a high content of various antioxidants. The change in antioxidant capacity of blood plasma 
was determined after the intake of different fruit servings. Both consumption of fruit salad and fruit beverage prevented against the decrease in antioxidant capacity, seen after the consumption of apples (chapter 7). The fructose in the fruit servings was found to increase the plasma uric acid concentration. The intake of compounds that are not identified as antioxidants (i.e. fructose) can cause an increase in antioxidant capacity by producing antioxidants (i.e. uric acid) during the metabolic pathway. Inducing an antioxidant by a non-antioxidant compound illustrates the complexity of determination of the antioxidant activity of compounds. In the design of antioxidant studies, the complexity of antioxidant activity should be considered.

In many clinical trials, healthy volunteers and patients use a high dose of antioxidant supplements. However, it should be realized that excessive intake of a specific antioxidant could disturb the equilibrium in the antioxidant network in healthy volunteers. A meta-analysis concluded that antioxidant supplements increased the mortality [1]. In patients with oxidative stress related diseases, this equilibrium is disturbed and antioxidant supplementation could be beneficial. It should be taken into account that reactive species can damage the cellular environment via different mechanisms, depending of the type of reactive species and the biological target. The intervention by scavenging antioxidants should be related to the reactive species and the biological target involved in the disease. If the biological target reacts fast with the reactive species, a fast reacting antioxidant is needed to prevent damage to the biological target. If a biological target reacts slowly with the reactive species, many antioxidants are able to protect the biological target. Also the reactive species involved have to be identified, since antioxidants react differently with the various reactive species. For example, the scavenging of singlet oxygen requires different properties from an antioxidant than scavenging a radical. The mechanism of antioxidant activity should be considered in the development of antioxidant treatments.

The complexity of antioxidant activity in vivo illustrates that the determination and accurate description of antioxidant activity, in a model or in man is still very challenging. 

$d x_{1}=-k_{j} \cdot x_{1}$
$d x_{2}=k_{0} w_{1}$ 


\section{Algemene samenvatting}
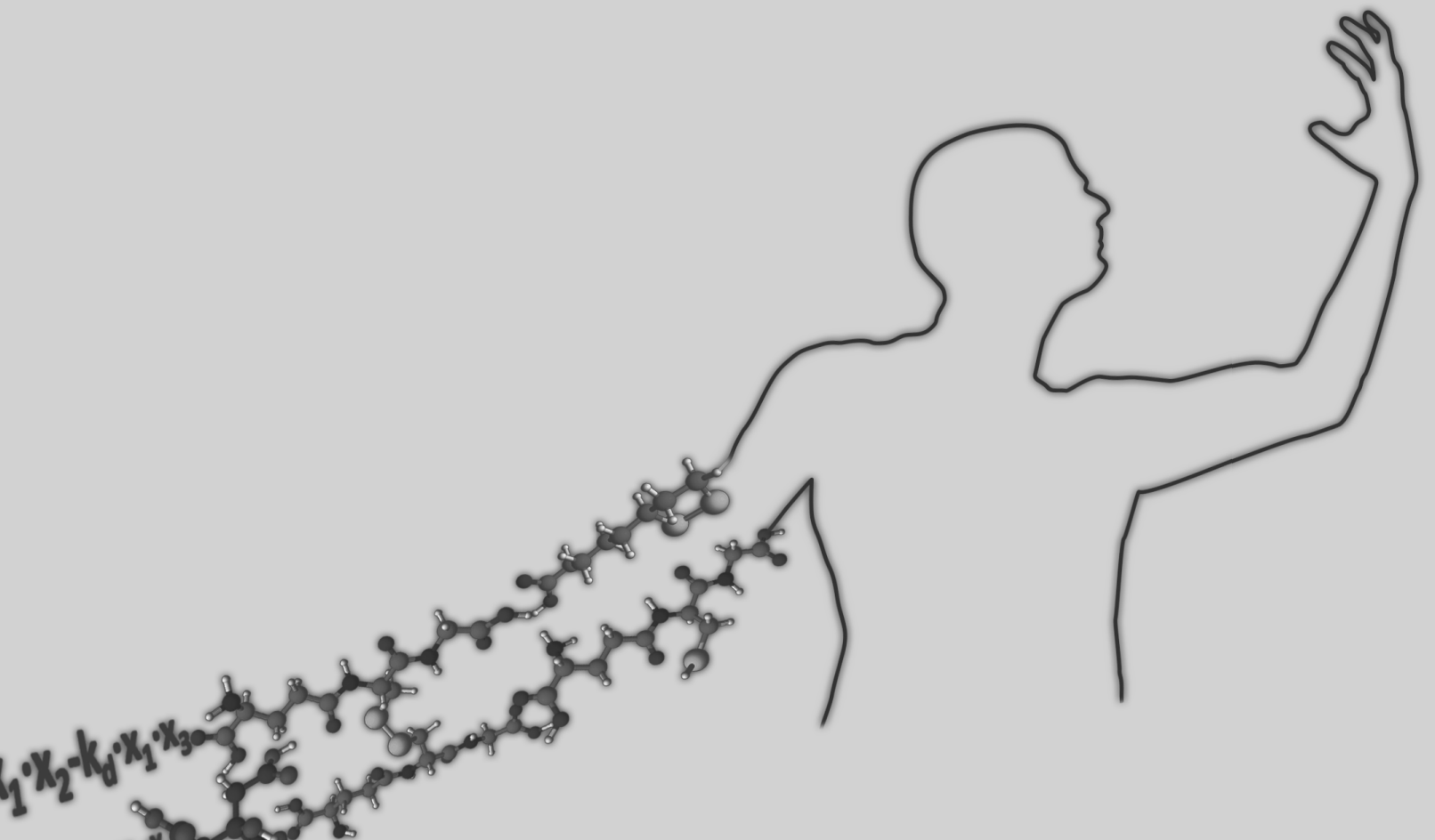

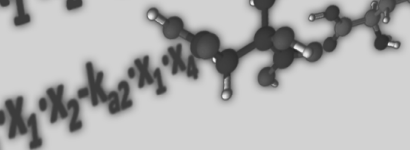



De mens kan niet zonder zuurstof. De zuurstof die wij inademen wordt vanaf de longen door het bloed naar alle cellen van ons lichaam gebracht. De cellen kunnen deze zuurstof gebruiken voor belangrijke processen. Eén van deze processen is de afweerreactie van het lichaam tegen schadelijke virussen en bacteriën. De afweercellen (witte bloedlichaampjes) beschadigen onder andere de celwand van virussen en bacteriën, waarbij ze zuurstof als wapen gebruiken. Hierdoor kunnen deze ongewenste binnendringers niet meer overleven en zijn ze niet meer schadelijk.

Gewoon zuurstof is niet reactief genoeg als wapen tegen deze micro-organismen. De afweercellen kunnen zuurstof omzetten in reactieve zuurstofdeeltjes (waaronder zuurstofradicalen). Deze deeltjes zijn reactief genoeg om met de celwand van de bacteriën te reageren, een reactie die ook wel oxidatie wordt genoemd.

Naast de afweerreactie zijn er nog veel meer processen in het lichaam waarbij reactieve zuurstofdeeltjes een rol spelen, zoals het regelproces van de bloeddruk, het gezond houden van de botten en de bloedstolling. De enzymen die zuurstof kunnen omzetten naar reactieve zuurstofdeeltjes komen dan ook in het hele lichaam voor.

Er kleeft echter ook een groot gevaar aan deze reactieve zuurstofdeeltjes. Ze zijn zo reactief dat ze met alles kunnen reageren. Wanneer ze op een verkeerde plek, een verkeerd tijdstip of in een te grote hoeveelheid gevormd worden, kunnen ze zoveel schade aanrichten dat de eigen cellen worden beschadigd. Dit kan tot gevolg hebben dat ziekten zoals COPD, diabetes type 2, leverfalen en kanker zich kunnen ontwikkelen.

Het lichaam heeft echter een verdedigingslinie tegen deze onbedoelde schade aan de eigen cellen, het antioxidant-netwerk. Een antioxidant is een stof die, op welke manier ook, schade aan de lichaamseigen cellen door reactieve zuurstofdeeltjes kan voorkomen. Eén van de manieren waarop een antioxidant kan werken is door de reactieve zuurstofdeeltjes weer terug om te zetten naar minder reactieve of niet-reactieve zuurstofdeeltjes; dit zijn de antioxidant enzymen. Een antioxidant kan ook zelf reageren met de reactieve zuurstofdeeltjes, zodat schade aan belangrijke moleculen voorkomen wordt; dit zijn de scavenging antioxidanten. Verder kunnen antioxidanten ook voorkomen dat reactieve zuurstofdeeltjes gevormd worden, door bijvoorbeeld het omzettingsproces van gewoon zuurstof naar reactief zuurstof te remmen.

Het eerste deel van dit proefschrift (hoofdstukken 2, 3 en 4) gaat over de scavenging antioxidanten. Dit soort antioxidanten kan relatief makkelijk via de voeding of in de vorm van medicijnen toegediend worden. Heel veel stoffen kunnen scavenging antioxidanten zijn. Sommige stoffen beschermen alleen beter tegen schade dan andere stoffen. Daarom probeert men door onderzoek de beste antioxidanten te identificeren. Door in een reageerbuis testen uit te voeren kan de activiteit van een antioxidant worden bepaald.

Over het algemeen wordt verondersteld dat de reactiesnelheid waarmee de antioxidant met het reactieve zuurstofdeeltje reageert de belangrijkste factor is die de activiteit van een antioxidant 
bepaalt. Daarom wordt geprobeerd de reactiesnelheid van een stof met een reactief zuurstofdeeltje te meten. Omdat de snelheden van de reacties van reactieve zuurstofdeeltjes zo hoog zijn, zijn ze echter niet direct te meten. Om toch de snelheid te kunnen schatten, wordt deze op een indirecte manier gemeten met zogenaamde competitie-experimenten. In deze experimenten moet de antioxidant een detector (een stof waarvan je via apparatuur precies de hoeveelheid kunt bepalen) beschermen door te reageren met de reactieve zuurstofdeeltjes. Er wordt dan gekeken hoeveel antioxidant er nodig is om $50 \%$ van de detector te beschermen tegen schade van de reactieve zuurstofdeeltjes. Van een goede antioxidant zal minder nodig zijn om 50\% van de detector te beschermen dat van een slechte antioxidant. De hoeveelheid antioxidant die nodig is om $50 \%$ van de detector te beschermen wordt de $\mathrm{EC}_{50}$ genoemd. Deze gemeten activiteit wordt dan gebruikt om de reactiesnelheid van de antioxidant met het reactieve zuurstofdeeltje te berekenen.

Het blijkt echter dat verschillende laboratoria op andere reactiesnelheden uitkomen, terwijl dit gelijk zou moeten zijn. Om er achter te komen waar deze verschillen vandaan komen is een competitieexperiment nagebootst met de computer. Met dit computermodel is aangetoond dat de gemeten activiteit sterk afhangt van de hoeveelheid reactieve zuurstofdeeltjes die gebruikt wordt. Als hier geen rekening mee wordt gehouden, is de gemeten antioxidant activiteit onjuist. Omdat het computermodel inzicht geeft in de mate van onderschatting of overschatting, kan de werkelijke reactiesnelheid beter bepaald worden. Ook zijn in hoofdstuk 2 een aantal aanwijzingen gegeven over hoe een competitie-experiment uitgevoerd moet worden om de antioxidant activiteit zo nauwkeurig mogelijk te bepalen.

In hoofdstuk 3 is gekeken naar een specifiek competitie-experiment met de detector deoxyribose en het hydroxyl radicaal als reactief zuurstofdeeltje. Met deze methode wordt in verschillende laboratoria de activiteit van een antioxidant bepaald. De laboratoria hebben echter grote verschillen tussen de uitkomsten voor dezelfde antioxidanten. Deze verschillen in activiteit bij dit experiment hebben alleen een andere oorzaak dan beschreven in hoofdstuk 2. Om dit experiment met deoxyribose en het hydroxyl radicaal te laten werken, zijn er meer stoffen nodig dan alleen het reactieve zuurstofdeeltje, de detector en de antioxidant. Zoals eerder beschreven zijn de reactieve zuurstofdeeltjes zo reactief (zeker het hydroxyl radicaal) dat ze met alle stoffen kunnen reageren. De stoffen die nodig zijn in dit experiment zullen dus, net als de detector, ook beschadigd worden door de reactieve zuurstofdeeltjes. Als hier geen rekening mee wordt gehouden, wordt de antioxidant activiteit systematisch onderschat. Deze onderschatting is relatief makkelijk te vermijden door rekening te houden met de aanwezigheid van die extra stoffen in het experiment. Hiervoor moet een aangepaste methode gebruikt worden om de reactiesnelheid van de antioxidant (de activiteit) te berekenen, die uitgelegd is in hoofdstuk 3 .

Een antioxidant moet snel met een reactief zuurstofdeeltje reageren om te voorkomen dat het reactief zuurstofdeeltje de cel beschadigt. Als echter kritisch wordt gekeken naar de eigenschappen 
die belangrijk worden geacht voor een antioxidant, is het de vraag of de reactiesnelheid wel het belangrijkste is. Een antioxidant hoeft alleen maar de cellen en delen van cellen te beschermen tegen de schade van de reactieve zuurstofdeeltjes. In hoofdstuk 4 is kritisch bekeken op welke manieren een scavenging antioxidant kan beschermen. Het blijkt dat de reactiesnelheid niet alleen de antioxidant activiteit bepaalt. Het is ook belangrijk met hoeveel reactieve zuurstofdeeltjes de antioxidant kan reageren. Om deze andere bepalende factor mee te nemen, is er een nieuwe manier voorgesteld om de antioxidant activiteit te beschrijven (hoofdstuk 4). Met deze nieuwe manier van beschrijven kan beter voorspeld worden hoe goed antioxidanten in het lichaam werken wanneer ze moeten beschermen tegen schade van reactieve zuurstofdeeltjes.

Een voorbeeld van schade door reactieve zuurstofdeeltjes is de leverziekte NASH (niet-alcoholische steatohepatitis). Bij deze ziekte is veel vet opgeslagen in de lever. Omdat levercellen niet gemaakt zijn om grote hoeveelheden vet op te slaan, gaan deze cellen kapot en komt er een ontsteking in de lever. Deze ontsteking zorgt weer voor extra reactieve zuurstofdeeltjes die nog meer schade veroorzaken aan de lever, met het gevolg dat de functie van de lever achteruit gaat.

Het is bekend dat in deze ziekte een enzym, CYP2E1, in verhoogde mate aanwezig is. Dit enzym, dat nodig is om de vetten te verbranden, kan ook reactieve zuurstofdeeltjes produceren. Als er meer van dit enzym aanwezig is, kunnen er ook meer reactieve zuurstofdeeltjes gevormd worden en kan er meer schade veroorzaakt worden. Het is echter nog niet bekend waarom dit enzym reactieve zuurstofdeeltjes produceert. Het zou kunnen dat de aanwezigheid van één specifieke stof dit enzym aanzet tot een grotere productie van reactieve zuurstofdeeltjes. Omdat de stof citraat meer aanwezig is in patiënten met NASH (hoofdstuk 5), is getest of citraat dit enzym stimuleert tot de vorming van reactieve zuurstofdeeltjes. Hoewel citraat wel meer aanwezig is in patiënten, zorgt het niet direct voor een verhoging van de productie van reactieve zuurstofdeeltjes. Welke rol citraat dan wel heeft in de ontwikkeling van de ziekte NASH is nog onbeantwoord.

Een andere manier om de lever in NASH te beschermen is door het enzym CYP2E1 af te remmen, zodat het minder of geen reactieve zuurstofdeeltjes meer vormt (hoofdstuk 6). Een enzym is een ingewikkeld molecuul dat heel kieskeurig is in de stoffen die het bij zich toelaat. Daarom moet eerst bepaald worden aan welke eisen de stof moet voldoen om in de buurt te komen van de plek in het enzym waar de reactieve zuurstofdeeltjes gevormd worden. Uit de verschillende stoffen die getest zijn, kan geconcludeerd worden dat de stof in ieder geval vetachtig moet zijn (lipofiel). Dit is de eerste stap in de ontwikkeling van een medicijn voor de ziekte NASH dat werkt via de remming van de aanmaak van reactieve zuurstofdeeltjes.

Veel antioxidanten komen uit de voeding. Voor hun werking moeten de antioxidanten ook opgenomen worden in het bloed. Via het bloed worden de antioxidanten overal in het lichaam gebracht en kunnen ze werken op plaatsen waar ze nodig zijn. Daar worden ze verbruikt en moeten ze weer aangevuld worden. 
Omdat fruit een belangrijke bron van antioxidanten is, is nagegaan wat het effect van het eten van fruit heeft op de antioxidantwaarde van het bloed (hoofdstuk 7). Hiervoor zijn drie verschillende porties fruit getest: een fruitsalade, appels en een fruitdrank. Merkwaardig is dat na het eten van appels de antioxidantwaarde van het bloedplasma daalt. Na de inname van een fruitsalade of een fruitdrank blijft de antioxidantwaarde van het bloedplasma op peil. De antioxidanten in de fruitsalade en de fruitdrank kunnen dus de daling van de antioxidantwaarde voorkomen. Om dit effect te zien was echter een correctie nodig. In fruit, en voornamelijk in appels, zit de stof fructose. Als fructose wordt opgenomen in de cellen, komt er langs een indirecte weg de antioxidant urinezuur in het bloed. Deze antioxidant komt dus niet uit de voeding, maar draagt wel bij in het op peil houden van de antioxidantwaarde van het bloed. Dit voorbeeld laat zien hoe ingewikkeld het is om antioxidant activiteit in het lichaam te bepalen.

Het bepalen van de antioxidant activiteit lijkt simpel. In dit proefschrift zijn echter fundamentele aspecten beschreven waar rekening mee gehouden moet worden in de bepaling van de antioxidant activiteit, om zo onjuiste conclusies te voorkomen. Dit laat zien dat het juist bepalen van de antioxidant activiteit, of het nu in een model is of in een mens, een hele klus is waaraan gewerkt wordt, maar die voorlopig nog niet geklaard is. 

$d x_{1}=-k_{j} \cdot x_{1}$
$d x_{2}=k_{2} w_{1}$ 


\section{Dankwoord}
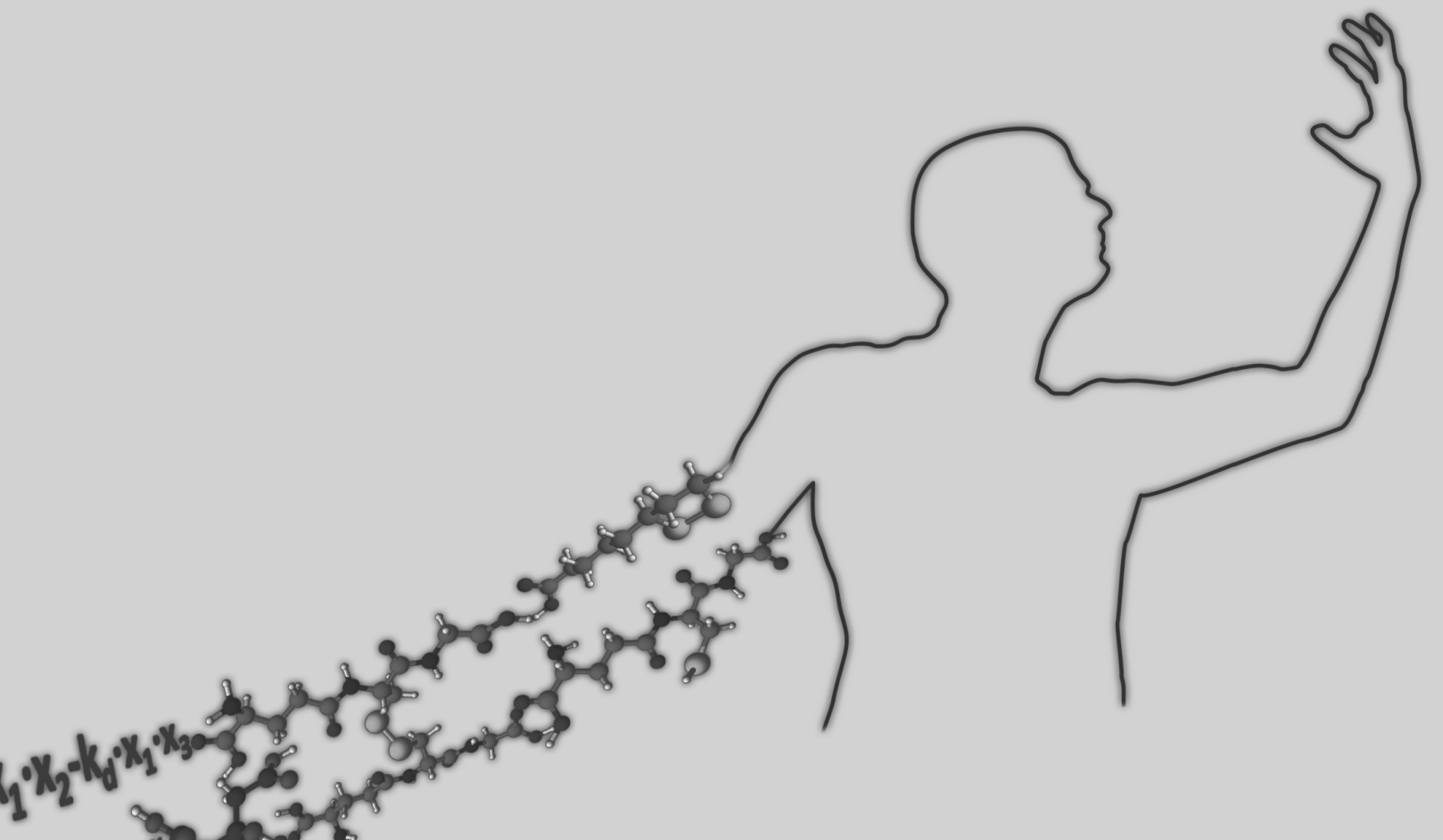

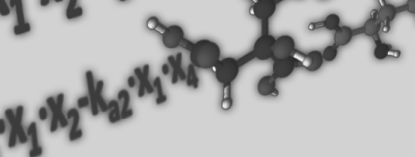



I said to the man who stood at the beginning of this road: "Give me a light that I may tread safely into the unknown." And he replied: "Go out into the darkness and put your hand into the hand of God. That shall be to you better than light and safer than a known way!"

Op deze weg heb ik niet alleen gelopen. Nu aan het eind van deze weg, en bijna aan het eind van dit boekje, kijk je terug en zie je iedereen die mee liep en mee hielp om mij bij dit eindpunt te brengen. Deze paar jaren zijn eigenlijk niet te beschrijven, maar deze laatste pagina's zijn een poging om mijn dank aan jullie in woorden te vatten.

Allereerst mijn promotor Aalt Bast en mijn co-promotor Guido Haenen. Jullie leiding begon al tijdens mijn afstuderen. De gezamenlijke besprekingen, eerst onwennig en zakelijk, werden al snel gezellige besprekingen over uiteenlopende onderwerpen. Van strips tot jaren '70 liedjes, van leesboeken en eten tot filosofie, politiek en geloof, alles kwam voorbij. Het onderzoek werd gedurende de rest van de week maar even besproken.

Aalt, dank je wel voor je enthousiasme en positieve kijk op dingen wanneer ik weer eens beren op de weg zag. Hoewel het je vaak niet gelukt is ze weg te praten, heb je me wel op het pad weten te houden.

Tijdens de soms pittige discussies (af en toe wederzijdse eigenwijsheid?) ben ik er ook achter gekomen dat het beta-jargon ook verschillende dialecten kent ('bedoel je met modelleren methode 1, 2, 3, 4a of 4b?'). Maar dit leidde wel tot weddenschapjes, waarvan er trouwens eentje nog open staat.

Guido, met jou heb ik afgeleerd om weddenschappen aan te gaan. Je hebt ze te vaak gewonnen. Dank je wel voor je enthousiasme, inzet, tijd, incasseringsvermogen en natuurlijk je 'functie' als vertaler tussen de verschillende beta-jargon dialecten. Het heeft heel veel pennen en papier gekost om mij de vertaalslag naar de biologie/biochemie te laten maken en de resultaten gepubliceerd te krijgen. De vele gedachte-experimenten hebben daar waarschijnlijk aan bijgedragen. Als deze experimenten daadwerkelijk uitgevoerd waren en opgeschreven, dan zou dit boekje niet meer door de brievenbus gepast hebben.

Aalt en Guido, jullie begeleiding in dit traject was en is onmisbaar.

Ger, dank je wel voor je medische kennis en je inbreng in het HepC en NASH onderzoek. Ook je hulp bij de praktische zaken was onmisbaar. Ik weet niet hoe vaak en waarbij ik je allemaal gestoord heb om een infuus (opnieuw) te prikken. Bedankt voor je geduld en flexibiliteit hierin.

De collega's van het onofficiële CLAIM (Centre of Laboratory Analysis, Information and Management) waren onmisbaar in dit traject. Jullie weten niet hoeveel ik van jullie geleerd heb. Van de basisbeginselen van het labwerk tot de limburgeringscursus met het talenpakket limburgs en maastrichts. Marc, Marie-José, Vanessa, Roger en Esther, dank jullie wel voor jullie gezelligheid, 
geduld en tijd die ik mocht 'claim'-en.

Marie-José, jou wil ik speciaal bedanken voor je hulp bij de HERO studie. Bij het vooruitzicht van drie verschillende analyses op 1072 samples zou mij de moed al in de schoenen gezonken zijn, maar jij had ze binnen no-time gedaan. In een woord geweldig.

A special word of thanks to my old room-mate and paranymph Shufan. Although we spend lots of time together, we still don't speak each other's language (yet). Among other stuff, the language problems caused a lot of fun. When necessary, we used hands, feet and even universal sounds to express ourselves. I still wonder why we never used a chinese-dutch/dutch-chinese dictionairy. Thanks for just being there, in all the ups and downs, excitements and frustrations. I'm glad that you are my paranymph to help managing my nerves. For the next promotion, it will be my turn to manage your nerves.

En dan mijn andere paranymf, Antje. Wat is het toch dat, zodra wij aan de praat raken, de tijd opeens veel sneller gaat? Het is al vaak gebeurd dat een simpel 'tot morgen' resulteerde in anderhalf uur later dan gepland thuiskomen. Hoewel het niet goed is voor mijn maag, hoop ik dat we vaker zulke gezellige avondjes zullen plannen. Dank je wel voor je hulp bij moeilijke situaties, maar ook bij de simpele dingen, zoals een zoektocht naar een centrifuge tijdens een klinische studie terwijl alle labs al afgesloten waren.

En dan mijn nieuwste kamergenootje, Merel. Terwijl ik dit schrijf, staart een lege stoel mij aan. De eerste maanden samen met jou op de kamer waren gezellig, en ik hoop dat die tijden snel weer herleven. Die lege stoel naast me is maar saai, die zegt niet zoveel terug.

Gertjan, dank je wel voor je hulp bij de ESR experimenten. En natuurlijk je suggesties voor mooie wandelingen in de Zuid-Limburgse omgeving. Nu de wandelschoenen bijna weer uit de kast kunnen komen, kan ik je suggesties gaan wandelen.

Saskia en Bertine, dank jullie wel voor jullie bijdrage aan het opzetten en uitvoeren van de HERO studie.

Natuurlijk wil ik ook de (ex)collega's Els, Hilde, Agnes, Daniëlle, Erik, Erik, Nuria, Max, en Liesbeth bedanken voor hun gezelligheid. Binnenkort weer tijd voor een TOX-uitje?

Ik ben ook grote dank verschuldigd aan 'mijn'studenten Elle, Bregje, Bertine, Katia, Anna en (hoewel niet officieel) Christiaan. Bedankt voor jullie inzet en flexibiliteit om op het laatste nippertje nog een paar experimenten te doen. Veel van jullie werk heeft het gehaald tot dit proefschrift en heeft de basis gelegd tot vervolgonderzoek. Ik wens jullie heel veel succes met het afronden van jullie studie en de stappen op het vervolgpad. 
Ook buiten de collega's was er steun en ontspanning. Jaap en Heleen, jullie weten precies welke fasen je allemaal doormaakt als AIO. Daarom was het geweldig om af en toe even te kunnen spuien en een steuntje in de rug te krijgen wanneer het nodig was. Jaap, leuk om elkaar zowel in de kerk als in de werksituatie tegen te komen. Ik hoop dat deze werkrelatie niet bij dit ene hoofdstuk blijft. En natuurlijk wens ik je ook heel veel succes met jouw laatste loodjes.

Jochem, met beiden een druk leven schiet het niet echt op om samen te musiceren. Je dwong me wel om weer achter de harp te kruipen en zo de nodige ontspanning te pakken. Nu hoop ik dat ik wat sneller die moeilijke stukken in mijn vingers en uit de harp krijg. Ooit moet het toch lukken om die Meditation, Tango en de Zwaan mooi uit te voeren.

En Gerrit, plaagmaatje en inmiddels dansmaatje, tijdens de loodzware loodjes kwam je humor (en jij) als geroepen. Je bracht de vrolijke noot (op een of andere manier, vraag me niet hoe) weer bij me boven. Ik hoop dat die vrolijke noot nog lang in de lucht blijft hangen, zonder dat we gek worden van elkaar (of anderen van ons).

En natuurlijk niet te vergeten Gert, Bieny, Mark, Esther, Piet, Wil, Marga, Arres, Hilde en verder iedereen van de gemeente voor jullie betrokkenheid en interesse. Ondanks dat ik er lang niet altijd in geslaagd ben om uit te leggen wat ik nu eigenlijk precies doe, bleven jullie meeleven.

Waar ben je zonder al je (schoon)broers en (schoon)zussen? Mariëlle, Geert, Hanneke, Corstiaan, Arjen, Hanneke, Sifra, Gerjon, zonder jullie steun, begrip, hulp, afleiding, advies, voorbeeld en gezelligheid was dit niet mogelijk. Paps en mams, jullie steun is echt onbeschrijfelijk. Jullie weten dat je de beste en mooiste dingen niet kunt zien, aanraken of beschrijven. Ze zijn alleen maar tastbaar met je hart.

\section{jiska}


$d x_{1}=-k_{j} \cdot x_{1}$
$d x_{2}=k_{0} w_{1}$ 


\section{Curriculum Vitae}
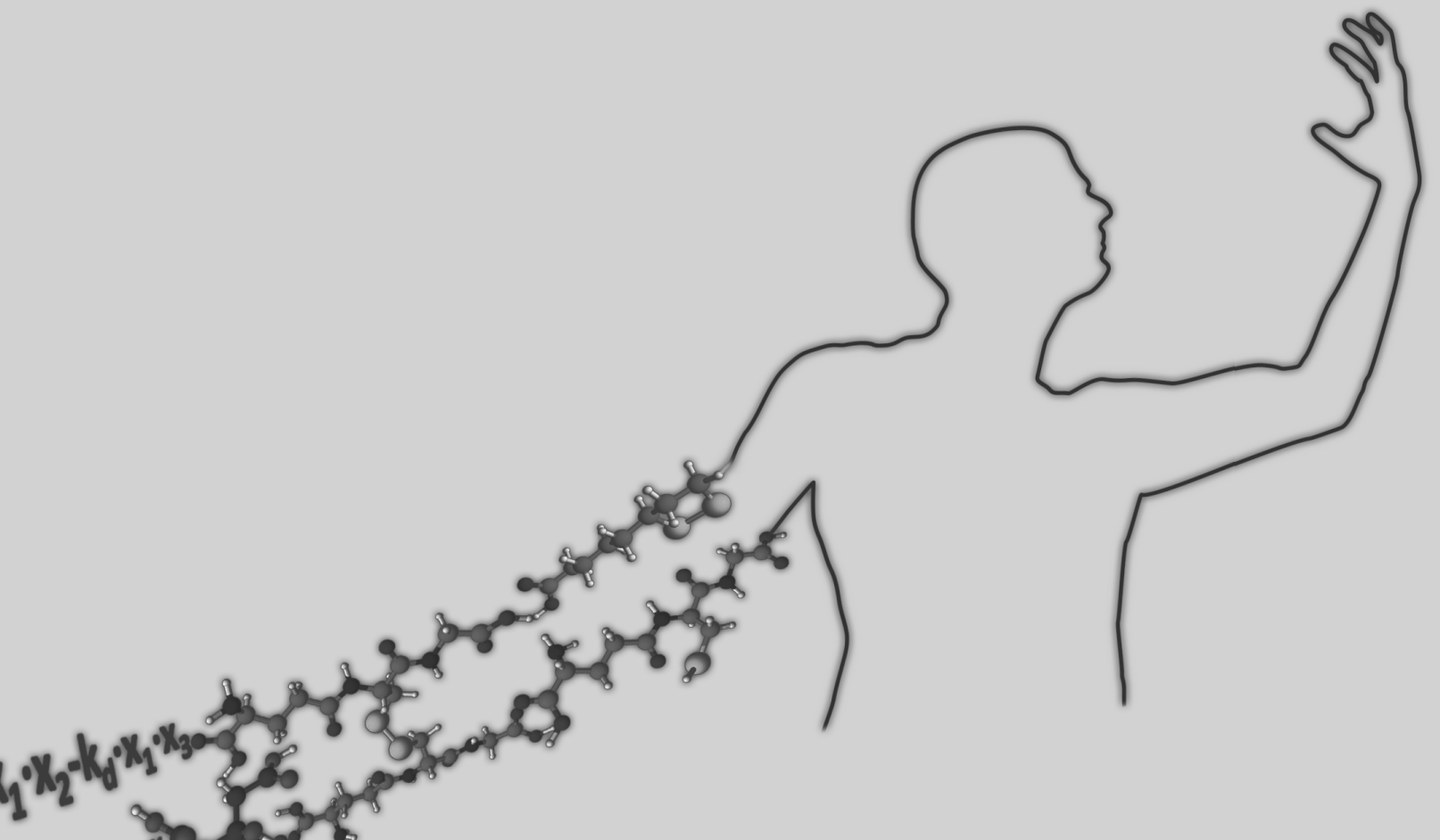

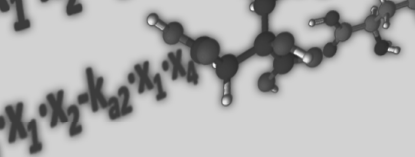



Jiska Mirjam Balk was born on April 27th 1981 in Mijdrecht, the Netherlands. In 1993, she started the pre-university education at Christelijk Streeklyceum (currently 'Het Streek') in Ede from which she graduated in 1999. In the same year she entered the one year trajectory ('basisjaar') at the Evangelische Hogeschool in Amersfoort. From 2000 to 2007 she studied biomedical engineering at the Technical University of Eindhoven. During this period, she was a member of the Dutch Christian Student association Ichthus, one year as treasurer. Her master thesis was supervised by prof. dr. A. Bast and dr. G.R.M.M. Haenen in the division of molecular bioengineering. She started her PhD study at Maastricht University on June 15t, 2007 on the subject of antioxidant activity under the supervision of prof. dr. A. Bast and dr. G.R.M.M. Haenen. The results of the research performed are described in the current thesis.

During her PhD study, she obtained the certificates of 'Safe Microbiological Techniques', 'Good Clinical Practice', and 'Laboratory Animal Science'. She is performing a phase IV clinical trial on the safety of ribavirin in Hepatitis $C$ positive patients.

After her PhD defence, she will continue to work at Maastricht University in the field of antioxidant research. 